AN EXAMINATION OF TECHNOLOGY-BASED FORMATIVE ASSESSMENT FEEDBACK AND THE IMPACT ON STUDENT LEARNING

A Dissertation
Presented to
the Faculty of the Graduate School
at the University of Missouri
In Partial Fulfillment
of the Requirements for the Degree
Doctor of Education
Dr. Barbara N. Martin, Dissertation Supervisor
brett A. Jacobs
by

May 2019 

(C) Copyright by Brett Jacobs, 2019

All Rights Reserved 
The undersigned, appointed by the dean of the Graduate School, have examined the dissertation entitled

\section{AN EXAMINATION OF TECHNOLOGY-BASED FORMATIVE ASSESSMENT FEEDBACK AND THE IMPACT ON STUDENT LEARNING}

Presented by Brett A. Jacobs a candidate for the degree of doctor of education

moreover, hereby certify that, in their opinion, is worthy of acceptance.

Dr. Barbara N. Martin

Dr. Sandy Hutchinson

Dr. Hayet Woods

Dr. Jason Morton 


\section{ACKNOWLEDGEMENTS}

First and foremost, I would like to convey my deepest and most sincere thanks to Dr. Barbara Martin. Dr. Martin has continually guided me throughout this process, and allowed me to grow as a student, professional and a person. Her love and guidance for all of her students is something that will never be lost or forgotten, as all of her students benefit from her knowledge, kindness, and warmth.

Each person who has accomplished this program, or any like it, understands it comes at a great sacrifice to many people in your life. I would not have been able to stay true to my course without the support of the members of Cohort 10. Particularly, those whom I spent countless hours during coursework at our local campus provided such thoughtful support and opportunities for growth. I feel so lucky to have had the opportunity to forge such meaningful relationships professionally, as well as many personal friendships, both of which will extend well beyond our time in this program.

Thank you to my mentor in life, my Dad, and the most selfless person I know, my Mom. I do not know what kind of man I would have become without your guidance and support. However, I am certain it would be a lessor version of my current self. Each day of my life I have gotten up with one goal: make you proud of me, my family, and my impact on the world, my ability to share kindness, love and generosity with others. You instilled those values in me from a young age. Everything I am is because of you, everything you passed on to us is forever your legacy. Thank you to my brothers who have always been my best friends. Having you two stand next to me and walk through life together has been a blessing. 
Thank you to my friends often referred to as my "family". I value every minute of our time together, and love that we can go days, weeks, months, and sometimes years without being able to gather, yet pick up right where we left off. I also appreciate your willingness to allow me to take a "leave of absence" these past few years as I took this journey. I look forward to our times of celebration, fun, and laughter again soon!

To my wife, Sandi. I could write an infinite number of pages and not adequately give the recognition and credit you truly deserve, or properly convey what you mean to me. Thank you so much for taking on so much so I could accomplish this life-long dream. Thank you so much for being the foundation of our family and loving me unconditionally. Your encouragement and insight were an instrumental part of this process. Ultimately, I could not have accomplished this, or anything, without you. Having you to share it with for the rest of our lives makes it all the more meaningful. LUM, my day, until 104.

Lastly, to my children Heidi, Connor, Stephen, and Kollin. I know you all have been on your own journey in life these past four years, navigating the world and facing challenges. I know my role is to guide and support you. Ultimately, I know you have given up a lot for me to be able to complete this program. For that, I am eternally grateful to you and hope you understand one day why daddy had to "go to class" or "go to work" in order complete his journey. My final hope for you is to know how much I love each and every one of you. How each and every thing I do is to elevate the quality of your lives and experiences. I live and breathe for you. I only hope to set a positive example for you so you can achieve whatever success in life you desire! Remember one 
thing; you are all amazing and special. You can do whatever you want, have whatever you desire, if you work hard enough and live life with a humble heart. 
Table of Contents

ACKNOWLEDGEMENTS .......................................................................... ii

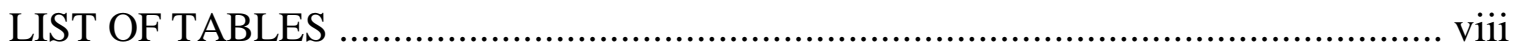

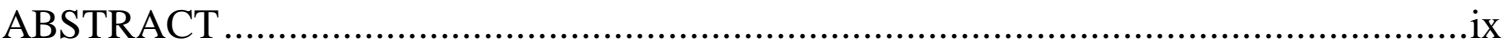

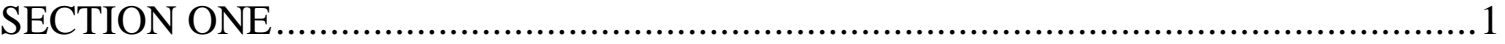

Introduction to the Background of the Study .......................................................2

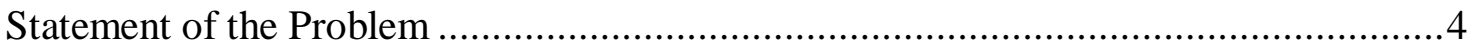

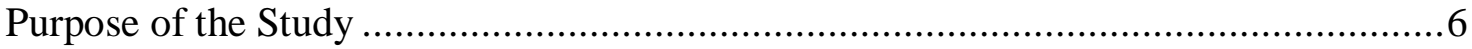

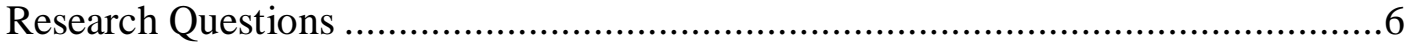

Conceptual/Theoretical Framework........................................................................

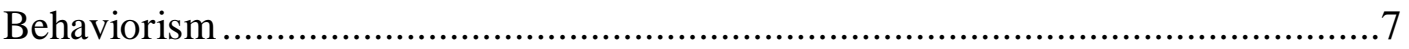

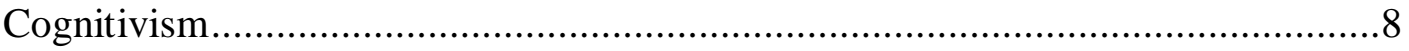

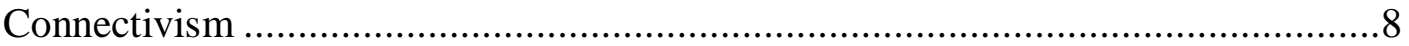

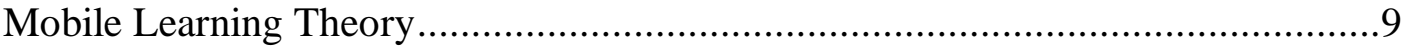

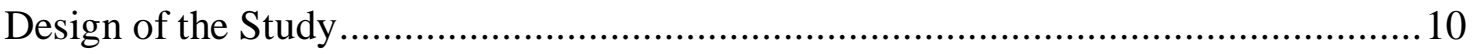

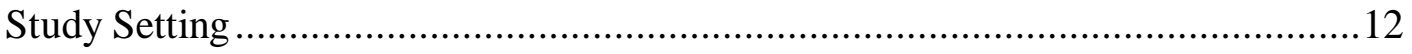

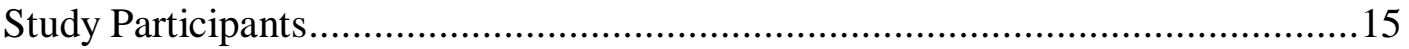

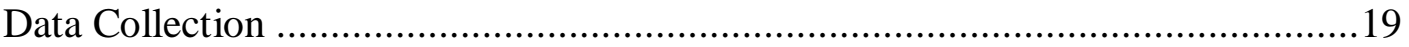

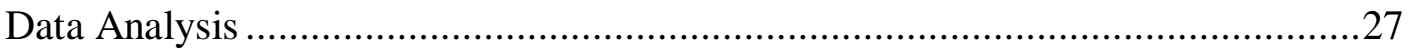

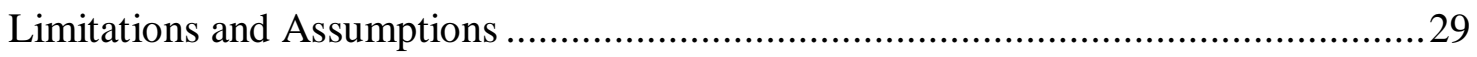

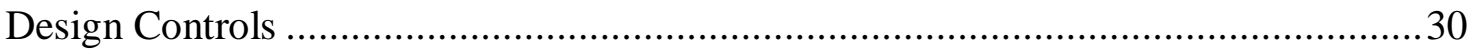

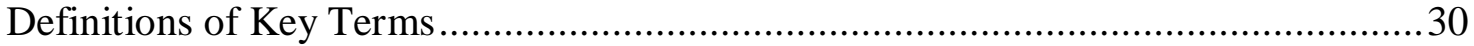

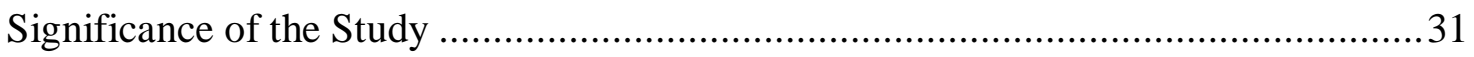

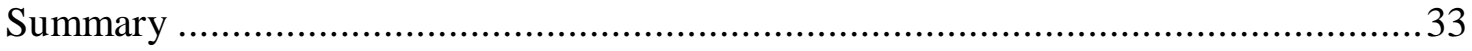

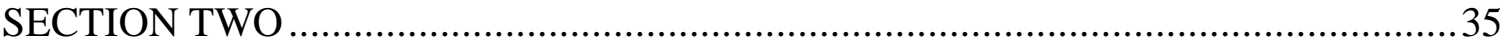

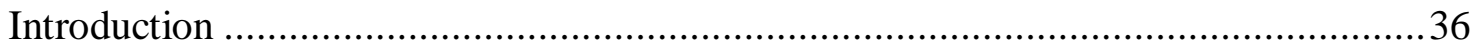

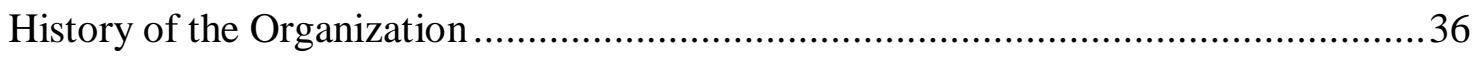

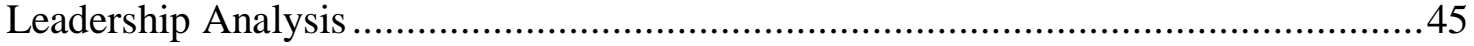

Implications for Research in the Practitioner Setting ......................................49

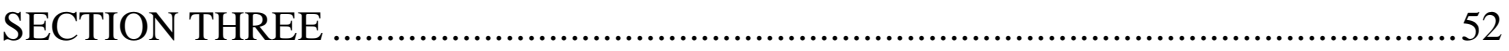

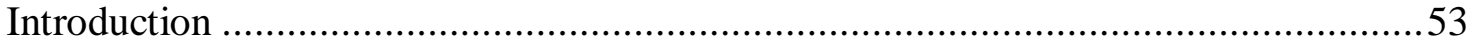


Learning Theory as a Theoretical Framework .......................................................53

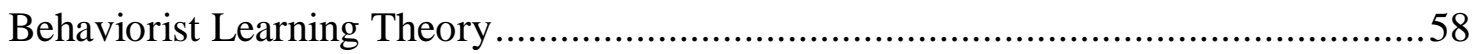

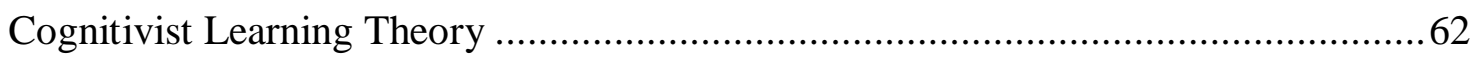

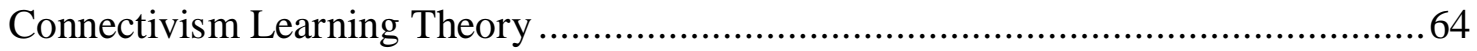

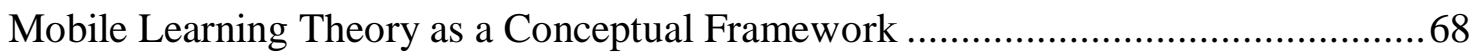

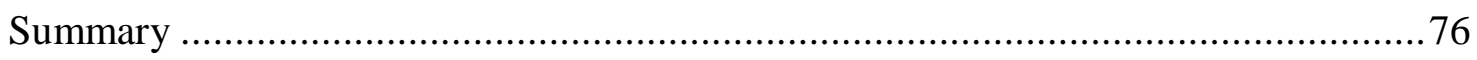

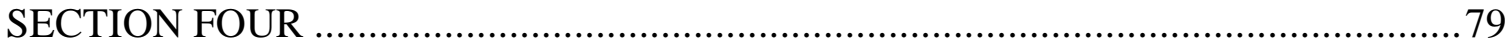

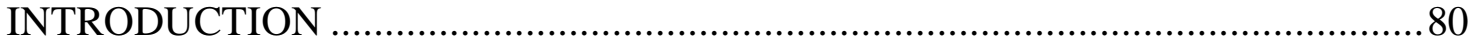

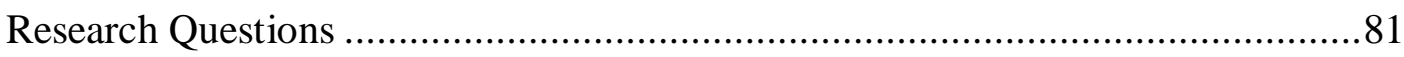

Connection to Conceptual/Theoretical Framework ....................................... 82

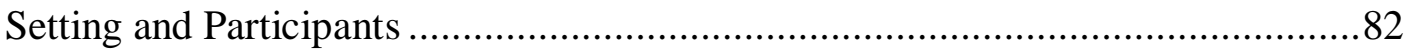

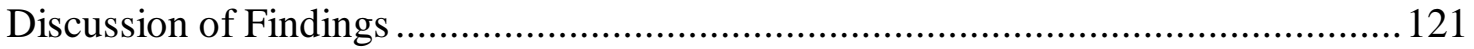

Theme: Impact of mobile learning on mastery learning .............................. 122

Theme: Impact of mobile learning on the quality and timeliness of data ............125

Theme: Lack of fidelity, training, and accountability in utilizing feedback data ..127

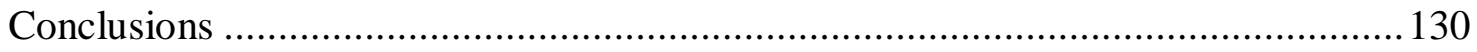

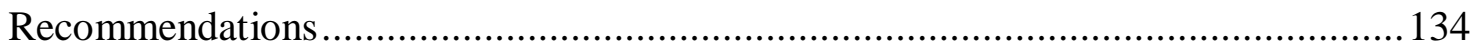

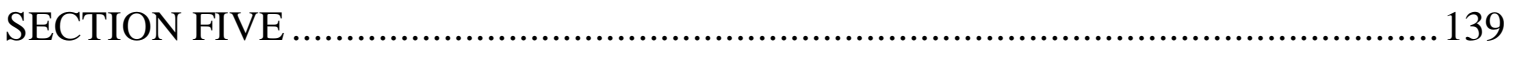

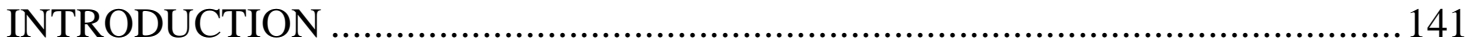

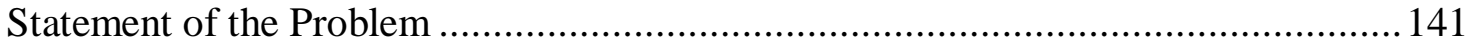

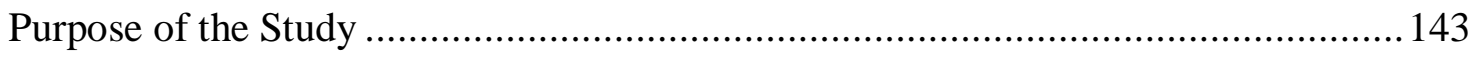

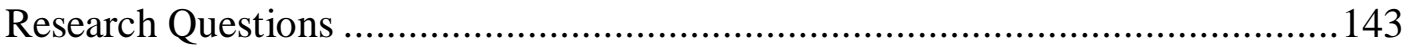

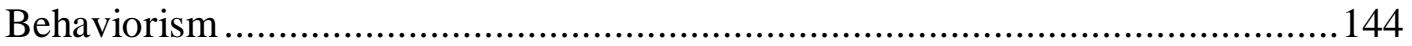

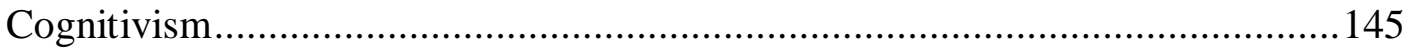

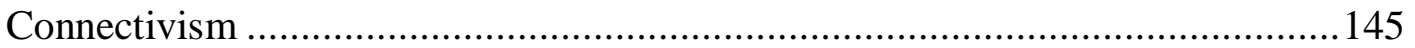

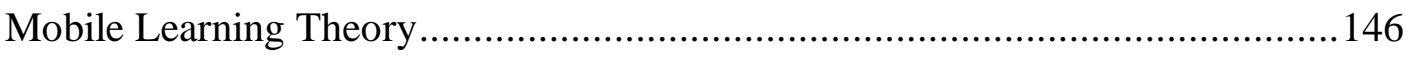

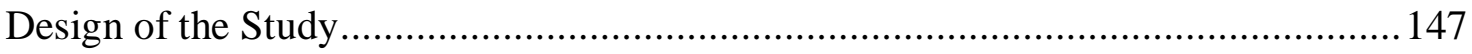

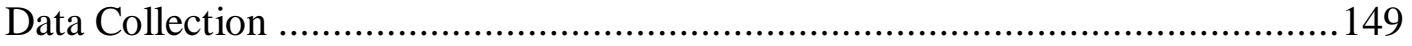

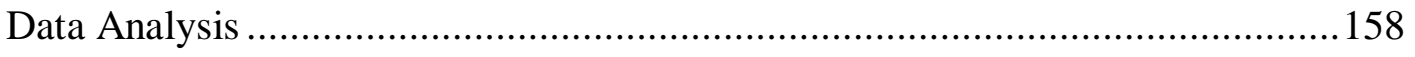

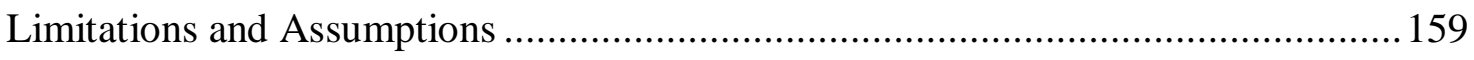

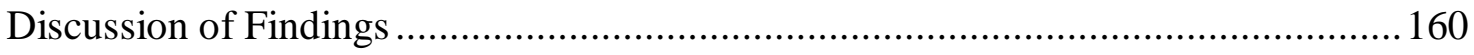

Impact of mobile learning on mastery learning ...................................... 160 
Impact of mobile learning on the quality and timeliness of data ....................... 164

Lack of fidelity, training, and accountability in utilizing feedback data.............. 166

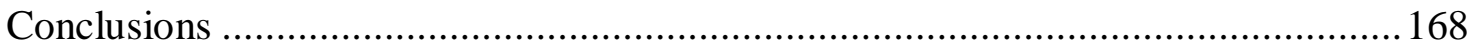

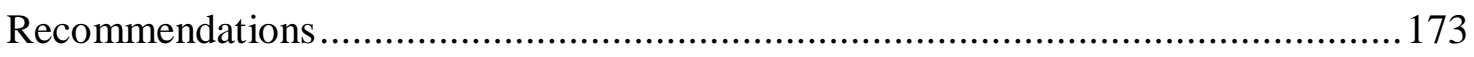

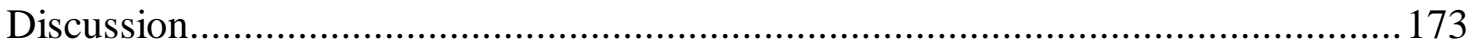

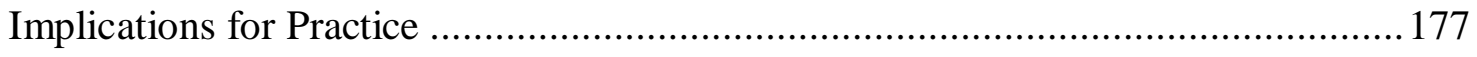

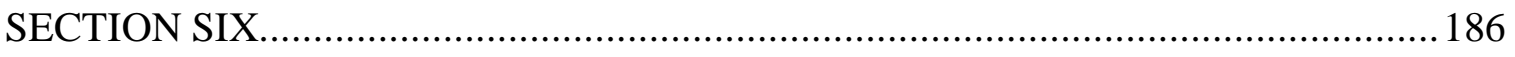

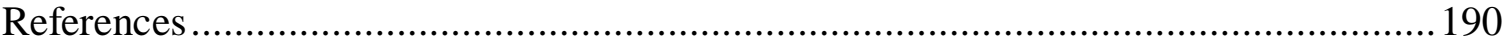

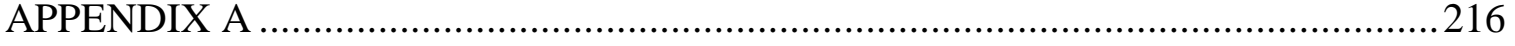

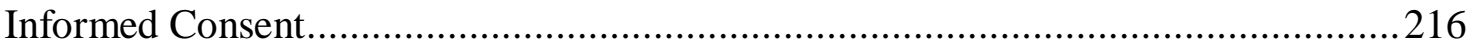

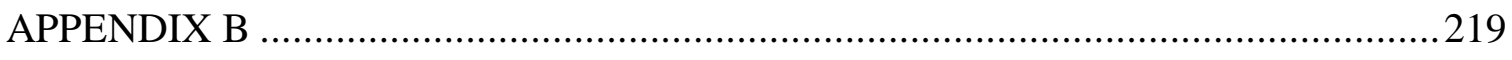

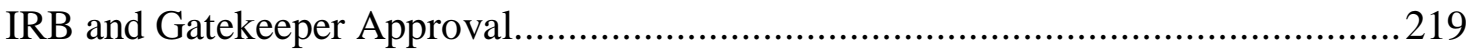

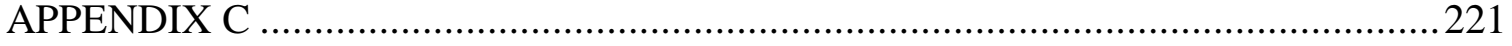

Informed Consent - Educator Participant ............................................................ 221

Informed Consent - Leader Participant ...................................................... 222

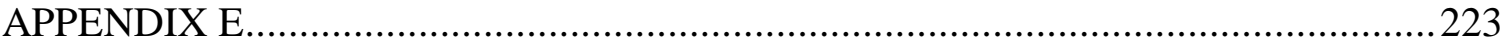

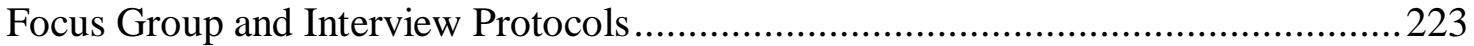

Focus Group Protocol: Classroom Teachers ................................................224

Focus Group: Curriculum and Instruction Team .........................................227

Interview Protocol: Building Administrator .........................................231

Interview Protocol: District Administrator ..................................................234

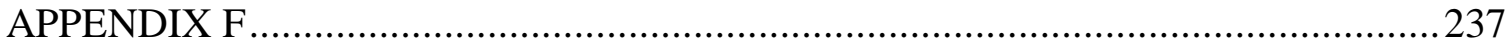

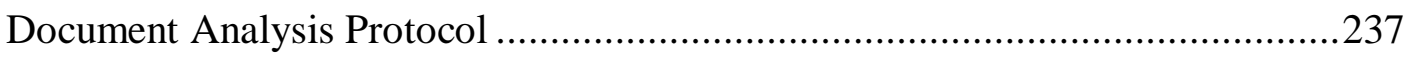

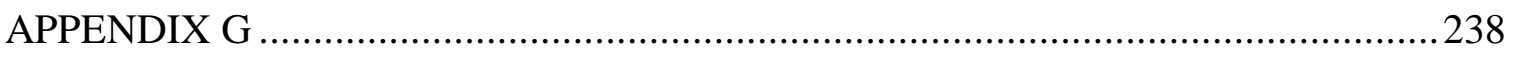

Observation Protocol Form................................................................ 238

APPENDIX H

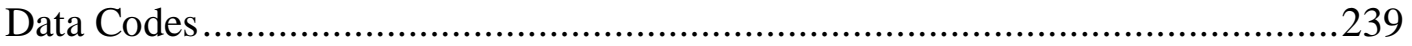

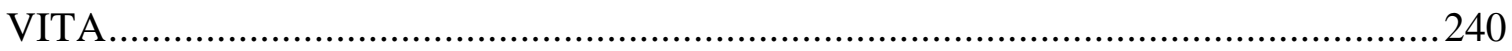




\section{LIST OF TABLES}

Table

Page

1. Description of Organization Structure of the Participants. 37

2. Description of Organization Structure of the Participants............................... 76

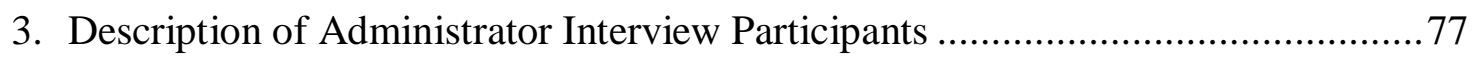

4. Description of Teacher Focus Group Participants .......................................... 78

5. Description of Specialist Focus Group Participants..................................... 79

6. Administrator Participants - Illustrative Quotes........................................... 81

7. IXL Focus Group and Interview - Illustrative Quotes ................................. 99

8. ALEKS Focus Group and Interview - Illustrative Quotes............................... 101

9. Schoology Focus Group and Interview - Illustrative Quotes.......................... 103

10. Timeliness and Quality of Feedback - Illustrative Quotes............................... 110 


\begin{abstract}
Although researchers have provided evidence to support the effectiveness of collaborative and cooperative learning (Magaña \& Marzano, 2014; Marzano, 2006) and embedding formative assessment within instruction (Black \& Wiliam, 2009; Wiliam, 2011), researchers (Chu, 2013; Hwang \& Chang, 2011; Sung, Chang \& Liu, 2016) further identified the need for research related to the impact of mobile devices on student learning. Purposefully selected for this study was the school district, as it is a highly innovative school district with regard to technology initiatives. Utilized during the process of collecting data for this case study, several sources were utilized. These included interviews with building and district administrators, focus groups with mathematics teachers and district instructional support staff, analysis of district and building documents, and observation of daily activities.

Qualitative analysis of interviews of district and site leaders, focus groups with instructional specialist and teacher focus groups, observation of meetings, and analysis of documents from the district resulted in three emerging reoccurring themes: The impact of mobile learning on mastery learning; The Impact of Mobile Learning on the Quality and Timeliness of Data; Lack of fidelity, training and accountability. Research from this study shows infusion of technology-based formative assessment applications has a positive effect on student learning. Further considerations when embedding technologybased formative assessment strategies within the teaching and learning cycle are to provide common training, resources, and accountability for implementation.
\end{abstract}




\section{SECTION ONE}

INTRODUCTION TO DISSERTATION-IN-PRACTICE 


\section{Introduction to the Background of the Study}

As the accessibility of technology devices and technology-based applications has increased, researchers (Andersson \& Palm, 2017; Chappuis, 2015; Pahomov, 2014; Wiliam, 2011) have attempted to make connections between practices within teaching and learning, and how technology can be used to enhance or deplete their effectiveness. Before what researchers (Johnson, 2014; Railean, 2017; Siemens, 2014) described as the digital revolution, educational standards, generically if not specifically, incorporated the preparation of students for success in the workforce. Moreover, preparing students to become productive, safe, and ethical decision-makers was also a prominent component of the educational design. While the infusion of technology as a matter of importance has triggered many technology-based initiatives, the goals for inclusion have been very broad.

Not previously addressed within the education standards was preparing $21^{\text {st }}$ Century workers within technology-based initiatives, as projections by economists have indicated the growing need for workers to have specific skills in technology (Alismail \& McGuire, 2015; Herman, 1999). When investigating technology within education, elements such as funding, program initiatives, and the introduction of specific technology standards indicate a unified belief within the educational community that technology is a necessary component of the educational process (Czaja, Charness, Fisk, Hertzog, Nair, Rodgers, \& Sharit, 2006; Martin, 2015). According to the United States Department of Education (2016), over \$200 million was allocated to improve the Education Technology State Grants program to improve instruction and personalized learning in high needs districts. Further, the National Education Technology Plan (2016) (NETP) indicated 
technology as a transformational learning tool that enhances and affirms teacher-student relationships, adjusts approaches to collaborative learning experiences, addresses gaps in learning, and provides an opportunity to adapt current learning experiences to meet the needs of all learners. The National Education Technology Plan (2016) further stated technology could enhance proven instructional methods and increase the potential of learning while consolidating the resources necessary to accomplish the same objectives. Because of this national movement in support of technology infusion, schools and school districts have responded with itemized plans to increase technological hardware and software, provide professional development for staff on effective technology-based instruction, and develop protocols for evaluating the effect of technology-based initiatives (Niederhauser \& Lindstrom, 2018; Schmidt-Crawford, Lindstrom \& Thompson, 2018).

Research on the effects of utilizing formative assessment strategies to provide feedback, and therefore, affect the overall learning of students, has been conducted in various forms. Research on how the use of effective formative assessment strategies increases student learning (Black \& Wiliam, 2009; Hattie, 2012; Rutheford, 2013; Wiliam, 2011), and research to support how technology integration can impact student learning (Jude, Kajura, \& Birevu, 2014; Martin, 2015; Puentedura, 2013; Romrell, Kidder, \& Wood, 2014) has been completed. Nonetheless, a reasonable gap exists between technology-based formative assessment and its impact on student learning. Specific to instruction, researchers (Marzano, 2006; Vaughn, Cleveland-Innes \& Garrison, 2013) stated utilizing a community of collaboration and inquiry with the purpose of reflecting on practice, utilizing social learning opportunities, encouraging 
dissertation in practice, B. Jacobs

group learning, and enabling self-reflection can lead to gains in student achievement.

Other researchers (Black \& Wiliam 1998, 2009; Wiliam, 2011) studied the origins and

effects of utilizing formative assessment strategies to determine the overall impact on the instructional process and overall student learning. Explicitly, Wiliam (2011) offered evidence regarding how the effective use of feedback and formative assessment can impact student learning followed by the consideration of how technology can be used to impact student learning through its use in formative assessment.

Similarly, research on differentiated instruction, cooperative and collaborative learning experiences, personalized learning, and utilizing formative assessment feedback to inform instruction continue to show positive results on overall student learning (Hattie, 2012; Magaña \& Marzano, 2014; Marzano, 2006, Rutheford, 2013). As such, it is this researcher's focus to determine whether technological tools enhance these strategies to increase the capacity of student learning. Therefore, this study will focus on exploring the impact technology has on learning as it pertains to increasing classroom efficiency, timeliness of feedback, collaboration and communication, and overall student achievement on specific learning objectives. Moreover, this researcher will reference examples of how formative assessment and technology infusion have been analyzed as isolated variables, while evidence exists to study the outcomes of formative assessment and technology simultaneously.

\section{Statement of the Problem}

As technology, and technology applications have increased in accessibility and functionality, with the allocation of resources growing at a faster rate than research can support. The acceleration of technology infusion under the premise of increased learning 
potential often occurred before the development of a professional development plan to account for teacher training. As such, Wiliam (2011) stated infusing technology alone would not yield the intended results of increased student achievement. While past efforts of providing classrooms with technological tools such as computer workstations and Smart Boards increased the level of technology access, researchers (Jude et al., 2014; Wiliam, 2011) maintained initiatives in technology have failed to increase achievement as such efforts merely modified the method of providing equivalent instruction. According to Faber, Luyten, and Visscher (2017), research on the effectiveness of technology-based tools and their impact on formative assessment feedback is lacking. Furthermore, researchers asserted that infusing technology in classrooms where teachers are trained to enhance feedback and modify instruction based on information gained should be further studied (Vaughn et al., 2013; Wiliam, 2011).

This problem of practice will explore the concept of examining technology as a tool to increase speed, accuracy, and quality of feedback. Additionally, this study will further examine the impact of technology-based formative assessment on teaching practices that ultimately influence student learning. The impact of technology on the effectiveness of proven educational practices is an area that is in need of further exploration. Specifically, as schools make strides in increasing access to technology and technological applications, the impact of technology-based formative assessment to modify instruction and provide alternate learning opportunities for students will provide useful information as to the significance of such initiatives. 
dissertation in practice, B. Jacobs

\section{Purpose of the Study}

Researchers defined formative assessment as the intentional gathering of information to inform and improve teaching within the process of learning (Black \& Wiliam, 1998, 2009; Cowie \& Bell, 1999; Wiliam, 2011). This researcher will analyze formative assessment grounded in technology-based tools and the impact on student learning within $7^{\text {th }}$ and $8^{\text {th }}$-grade mathematics. The researcher will examine how the frequency of feedback within instruction impacts student performance through assessments designed to measure student success (Romrell et. al., 2014; Wiliam, 2011). Further, this researcher will explore the technology-based applications to identify which significant factors impact student results accounting for frequency, informal collaborative learning experiences, and instructional efficiency (Looi et. al., 2009). Moreover, the researcher will study the impact of technology-based formative assessment applications on preparation and collaboration, and whether applications affect student performance related to designed assessments (Hwang \& Chang, 2011; Nicol \& Macfarlane-Dick, 2006; Reeves, Gunter \& Lacey, 2017).

\section{Research Questions}

The following research questions guided the present study:

1. How do technology-based tools and applications impact the way teachers gather, analyze, and utilize information obtained from formative assessments to impact student learning?

2. How does the professional development and training of teachers and administrators specific to technology-based tools and applications (IXL, ALEKS, 
dissertation in practice, B. Jacobs

Schoology, or other applications) impact the way teachers adapt instructional learning opportunities?

3. How do technology-based formative assessment resources impact the frequency and quality of feedback utilized by teachers to enhance teaching and learning?

\section{Conceptual/Theoretical Framework}

This researcher will examine the theoretical framework of learning theory to determine the impact of technology on student learning. Behaviorism, Cognitivism, Connectivism, and Mobile Learning (MLT) theories will be utilized to assess whether learning occurs independent of, or in conjunction with, technology-based and social constructivist methodology. Infusing technology within learning experiences will be examined utilizing the SAMR model, which depicts four levels of technology integration (Jude et. al., 2014; Puentedura, 2013; Romrell et. al., 2014). As previously stated, the purpose of this research is to determine whether technology makes an impact on student learning through the stakeholders perceptions. The SAMR model provides a template for evaluating the effectiveness of technology incorporation and allows for analysis of the impact of technology on learning through the frameworks.

\section{Behaviorism}

When considering the behaviorist approach, the intended function of technology integration results from changing the responses of students by introducing new stimuli. (Ally, 2004; Boghossian, 2006; MacCallum \& Parsons, 2016; Ormrod, 2012). As variables in this case, introduced are technological applications, since learning occurs as a function of reinforced responses based on observable behaviors. Behaviorism supports learning as a sequence of events independent of internal processing or reflection (Ally, 
dissertation in practice, B. Jacobs

2004). As such, behaviorism-learning theory met the substitution level criterion, where technology-based learning activities existed as a function of replacing traditional learning experiences (Puentedura, 2013; Romrell et. al., 2014).

\section{Cognitivism}

Explored as an alternative to the behaviorist learning theory was cognitivism, as researchers indicated it better explains how learners process information as a part of teaching and learning (Berliner, 2006; Bransford et. al., 2000; Yilmaz, 2011). Tenets of cognitive learning allow for comparative analysis of prior knowledge and construction of new information. Learners are required to use logic to make meaning of the learning experience, and thus commit new information to memory as a rote response (Berliner, 2006). Cognitivism provides a deeper venue for exploring technology than behaviorism by incorporating variables such as feedback and social interaction. However, cognitivism fails to explain learning experiences beyond the non-technology enriched activity.

\section{Connectivism}

Connectivism learning theory evolved in response to the need to explain how technology changes the process of learning (Barry, 2013; Kizito, 2016; Ozan \& Kesim, 2011; Siemens, 2014). One primary principle of connectivism is that technology redefines knowledge to be an interface with information (Bell, 2011; Downes, 2008; Siemens, 2014). Traditional theorists define knowledge as information processed, transferred, and committed to memory, thus making it a tangible set of acquirable facts (Boghossian, 2006; Conradie, 2014; MacCallum \& Parsons, 2016). Connectivism defines knowledge as the ability to access information through connections made possible using technology (Anderson \& Dron, 2011; Kop \& Hill, 2008). Technology 
within connectivism provides a pathway to knowledge and serves as a tool for increased access to information (Kizito, 2016; Siemens, 2014). As such, connectivism-learning theory places emphasis on creating pathways to information that can be continually accessed and updated in real time (Siemens, 2014). Although connectivism provides a venue to connect technology and learning, a gap still exists between its principles and those of formative assessment feedback.

\section{Mobile Learning Theory}

Mobile learning theory (MLT) utilizes technology to transform learning, creating instant and expansive opportunities within and outside of the classroom for learning to occur (Romrell et. al., 2014, Sharples, Taylor, \& Vavoula, 2007). Technological tools and applications can be utilized to substitute, augment, modify, or redefine traditional learning activities in a way that maximizes the experiences for effective instructional design (Puentedura, 2013; Romrell et. al., 2014). Researchers also asserted through use of technology-based tools that learners could obtain information in different contexts, thus allowing for a more effective transfer and commitment of information to memory (Hwang \& Chang, 2011; Pachler et. al. 2010; Puentedura, 2013; Romrell et. al., 2014). MLT emphasizes incorporating feedback and formative assessment as a part of the instructional process (Burns, Klingbeil, \& Ysseldyke, 2010; Dyson \& Frawley, 2016;

Lee, Feldman, \& Beatty, 2012). This researcher determined MLT as the conceptual framework that allows for examining the augmentation or redefinition of learning activities, which researchers (Puentedura, 2013; Romrell et. al., 2014) described as expanding learning experiences beyond what is capable without technology. 


\section{Design of the Study}

Merriam and Tisdell (2016) identified an essential component when designing qualitative research as one to determine how to design the study because of the theoretical framework in connection to the purpose of the study. Researchers within a basic qualitative study gather information related to how people interpret experiences, construct their worlds, and the meaning they attribute to those experiences (Creswell, 2014; Merriam \& Tisdell, 2016). Within this basic qualitative study, the researcher utilized a case study design as outlined by Creswell (2014), Merriam, and Tisdell (2016). Researchers (Baxter \& Jack, 2008; Creswell, 2014; Merriam \& Tisdell, 2016) described a case as an analysis of information gathered from multiple sites and a variety of participants visited, observed, or interviewed. Further, Merriam and Tisdell (2016) outlined the function of a case study is to analyze a phenomenon within a bounded system, such as a person, program or event. In this case study, the case is identified as how technology-based formative assessment feedback impacts student learning in the three selected sites within the District. In this study, the researcher will identify teachers, principals, and district personnel with explicit information pertinent to the impact of technology-based formative assessment within the sites and District. Analyzing data collected through interviews, focus groups, observations, and document analysis provides evidence when identifying and interpreting the meaning within recurring themes (Krueger \& Casey, 2014; Merriam \& Tisdell, 2016). The use of a bounded case study will allow the researcher to solicit data to inform potential conclusions.

According to Baxter and Jack (2008), once a case study and its boundaries are established, researchers should next consider the type of case study to conduct, selecting 
a case design guided by the purpose of the study. Yin (2003) outlined designs of case studies to be: 1) explanatory - creating causal linkage between program implementation and program effects, 2) exploratory - where interventions have no clear set of outcomes, or 3) descriptive - to describe a phenomenon and the real-life context in which it occurred. This study will use an explanatory case study design to determine whether the phenomenon, integrating technology within formative assessment, creates an effect of increased student learning. As such, the goal of the researcher is to rely on the views of the participants utilizing broad, generalized, questions allowing for discussion or interaction to construct meaning (Creswell, 2014). Crotty (1998) asserted meaning is constructed, not discovered, within the interpretation of an experience or social interaction. Therefore, the researcher will operate within the paradigm of social constructivism, the belief that, "individuals seek understanding of the world in which they live and work" (Creswell, 2014, p. 8).

Social constructivism, according to researchers (Amineh \& Asl, 2015; Creswell, 2014), is a theory of knowledge where knowledge is constructed jointly and in coordination with others to create meaning of a constructed reality. Within social constructivism, the essential components of the paradigm focus on the production and construction of knowledge as a group, where learners construct knowledge and meaning of experiences with other members of the learning community (Doubleday et. al., 2015; Vygotsky, 1978). Schunk (2012) asserted teaching from a social constructivist perspective emphasizes strategies that promote learning with others, such as reciprocal teaching, problems-based instruction, and peer collaboration. Teachers play the role of 
the facilitator and allow for the construction of understanding by individual learners (Amineh \& Asl, 2015).

Creating an environment of interaction, such as increasing the role of technology, as a function that supports learning inherently increases the opportunity for teachers and students to construct meaningful learning opportunities beyond traditional core skills (Kivunja, 2014). Social constructivism provides a paradigm of learning for teachers, as developing strategies for meaningful teaching and learning with technology is dependent on the collective experiences of the group. In this case, teachers play the role of the learner, reflecting upon benefits and detriments of technology-based formative assessment with each experience, therefore constructing their own inferences and learning connected to its impact on student learning. In addition, this study will create an opportunity for participants to discuss collectively their experiences within teaching and learning related to technology-based formative assessment and its impact on student learning. The constructed knowledge of participants within this qualitative study will allow the researcher to triangulate information, present emerging themes and formulate conclusions relevant to the research questions (Merriam \& Tisdell, 2016; Yin, 2003).

\section{Study Setting}

This researcher selected a second tier suburban school district (District) located outside of Kansas City, Missouri, as the case for this study. Purposefully selected for this study was the school district, as it is a highly innovative school district with regard to technology initiatives. One example is the district constructed a site where the district collaborates with a state college to provide career and technical education in conjunction with post-secondary degree programs. Most recently, this facility was nationally 
dissertation in practice, B. Jacobs

recognized with the 2018 Learning by Design Award, an award given to the learning space with the best educational design. Located within this site is a program designed to provide alternative technical education to students from District and area school districts, featured in Kansas City Public Television's School of the Future series in 2018. Other recognitions for District include receiving Missouri’s Distinction in Performance Award each year of its existence, identified by BusinessWeek magazine as Missouri's Best Place in America to Raise Kids, and by Money Magazine as being seventh in the nation on the list of Best Places to Live.

In addition, the District has a reputation for allocating resources in support of technology incorporation and infusion. In 2013, District adopted an initiative that provided technology devices to each student, consequently increasing the availability of technology-based tools and applications to teachers and students. The program was implemented to enhance instruction, collaboration, and provide an additional learning tool for students and teachers. Further goals of the program were to increase the digital citizenship and $21^{\text {st }}$ century skills identified by the International Society for Technology in Education (ISTE) standards. Therefore, this researcher selected the District because of its reputation for innovation, commitment to technology integration, and accessibility of resources and training directly related to the study.

Within the case, the researcher utilized three sites specific to $7^{\text {th }}$ and $8^{\text {th }}$ grade mathematics instruction. The District is composed of three middle schools, containing approximately twenty-eight certified teachers employed to implement curricular programs and provide mathematics instruction and assessment to approximately 2,850 middle school students (Department of Elementary and Secondary Education, 2018). 
Each middle school site has a three-person administrative unit, including a Principal and two Assistant Principals. The three-person administrative team is the direct supervisor of the teachers, evaluating and facilitating instructional effectiveness and growth. A districtlevel secondary curriculum specialist, who works concurrently with other content specific coordinators, and reports to the Director of Curriculum and Instruction, develops curriculum. The curriculum director and secondary specialist coordinators collaborate with teachers and building administrators to implement the CSIP and meet state-specified standards within student achievement. The district employs staff specific to technology infusion, technology specialists, to foster and create opportunities within instruction through the means of technology-based applications and resources aimed at increasing student learning. In addition to professional development provided by technology specialists, instructional coaches provide training and development for teachers that incorporates, but is not exclusive to, technology integration.

The district utilizes shared human resources to support teachers throughout the district including curriculum, instructional, and technology specialists, in addition to leaders at the district level specifically designed to increase, enhance, and support technology infusion and integration. District secondary (7-12) courses are aligned vertically to standards outlined by the Department of Elementary and Secondary Education (DESE). The district supports each school with comparable staff, instructional objectives, and financial resources. In addition, District provides professional development to mathematics teachers and offers collaborative opportunities for teachers to reflect on the effectiveness of practice. Specifically, this researcher purposefully selected the District, and the sites within, to conduct this inquiry based on: 1) the 
dissertation in practice, B. Jacobs

District's distinction in performance, 2) initiatives directly related to technology incorporation, 3) the expertise and knowledge of the teachers selected, 4) the human resources allotted to professional development specific to technology tools and applications, and 5) the ability to triangulate data collected from a variety of sources.

\section{Study Participants}

As previously outlined, this researcher conducted a case study of the District, utilizing three middle school sites, to examine the effects of technology on formative assessment practices. Further, the information gathered from participants was analyzed through a social constructivist lens, allowing participants within the focus group to construct meaning of their shared experiences (Doubleday et. al., 2015; Kivunja, 2014). Analysis of data through the social constructivist perspective supports the rationale for the case study. This process allowed participants to construct meaning within shared experiences and ultimately provide data related to the impact of technology-based tools and applications on student learning.

As was the purpose in selecting District and the chosen sites within, participants within this study provided depth and richness to the study. Specifically, the allocated resources to technology initiatives and teacher development within technology instruction provides credibility to selected participants when evaluating the effects of technologybased formative assessment. Teachers, administrators, and district level employees have been immersed in technology integration through increased access and training, thus cultivating their environment, knowledge, and experiences. Kreuger and Casey (2014) asserted three key factors when selecting participants for focus groups, such as identifying the purpose of the study, knowledge and accessibility of potential 
dissertation in practice, B. Jacobs

participants, and the budget for the study. Further, the purpose of selecting an appropriate sample is to achieve saturation, or gathering data until it ceases to provide new insights or information to the study (Charmaz, 2009; Creswell, 2014; Merriam \& Tisdell, 2016). This researcher utilized purposeful sampling to select the focus group and interview participants, identify specific documents for review, and gather data designed to answer the research questions within this case study based on the relevance of information they can provide (Creswell, 2014; Merriam \& Tisdell, 2016; Patton, 2015; Yin, 2011). Merriam and Tisdell (2016) further elaborated the function of purposeful sampling is to ensure the researcher has access to the participants who have the most information to contribute to a subject. Although a random sample can reduce bias, researchers asserted random sampling might be a more appropriate choice when the represented group is large enough to distribute normally the attitudes and opinions of the group (Creswell, 2014; Krueger \& Casey, 2014).

The researcher communicated with the District Instructional Team (DIT) to communicate the criterion for participant selection. Mathematics teacher focus groups consisted of participants from the district where each met initial criterion of being district employees of at least three years, or have at least five years of teaching experience within $7^{\text {th }}$ or $8^{\text {th }}$-grade mathematics. Krueger and Casey (2014) asserted this process for selecting participants reduces bias and increases the quality of the data collected. The participants of three focus groups were comprised of $7^{\text {th }}$ and $8^{\text {th }}$-grade middle school mathematics teachers from each site ( $\mathrm{n}=15$ participants). Mathematics teachers were selected based on the expertise they possess in mathematics instruction and their specific knowledge of teaching, learning and assessment practices as it relates to the study. 
Mathematics teachers within this study hold at least a bachelor's degree in mathematics education and have experience that ranges from three to 29 years. Out of the 25 potential participants, 22 hold a master's level degrees or above, including one holding an educational specialist degree. According to the Department of Elementary and Secondary Education (2018), a bachelor's degree in a content area makes each participant highly qualified to teach mathematics. However, holding a non-content area master's degree does not inherently make one more qualified to teach a content area.

An additional focus group comprised of the district-level participants $(n=6$ participants) was formed to gather information, potentially including the district curriculum, technology, and instructional specialists. District level staff members were chosen for this focus group based on their qualifications within the district in supporting teaching and learning, expertise in technology integration, knowledge of technology integration and professional development at the three chosen sites, and knowledge of technology-based formative assessment tools utilized within District. Specifically, curriculum specialists were selected due to their knowledge of the learning standards, curriculum and instructional pedagogy, and knowledge related to formative and summative assessment design. Technology and instructional specialists were selected because of their role in professional development, technology infusion, and instructional support to the teaching and learning process within $7^{\text {th }}$ and $8^{\text {th }}$ grade mathematics classrooms. Participants within this focus group hold at least a bachelor's degree and certification in one or more educational areas. These participants have teaching experience that ranges from 12 to 19 years teaching, and experience in district level 
instructional support ranging from one to seven years. Each of the 6 participants held a master's level degree or above within an educational field.

In addition to focus groups, interviews $(n=5)$ conducted with middle school building administrators ( $\mathrm{n}=3$ participants), and district-level administrators $(\mathrm{n}=2$ participants) allowed the researcher to triangulate information to formulate conclusions (Lincoln \& Guba, 1985; Yin, 2003). District level participants included the Director of Instructional Technology and the Associate Superintendent of Academic services. These participants were purposefully selected based on their expertise related to instructional technology, formative assessment feedback, data analysis or knowledge of content area specific to the study. Building principals from each site were selected in order to triangulate data collected from focus groups in each site, utilizing their observation of teaching and learning experiences, knowledge of the frequency and effectiveness of technology-based formative assessment applications, and reflections on the effects of district initiatives related to technology. The researcher selected District leaders within the instructional technology department, such as the Director of Technology, based on their knowledge of the district technology plan as it relates to the ISTE standards, their ability to provide evidence related to the effects of technology integration initiatives, as well as their expertise regarding how the district supports instruction and assessment utilizing technology tools and applications. These participants have teaching experience that ranges from five to 12 years teaching, and experience in administration ranging from six to 15 years. Each of the participants has completed the requirements for principal certification as required by the Department of Elementary and Secondary Education 
(2018). Out of the five administrative participants, all hold a master's level degree or above, including three who possess doctoral degrees.

This study was designed to collect data from the middle school mathematics instructors, curriculum specialists, instructional and technology support staff, and building and district administrators. Gathering data from these purposefully selected sources allowed this researcher to triangulate information, which researchers (Merriam \& Tisdell, 2016; Yin, 2003) define as crosschecking data from a variety of places or people, or following up on findings at a different times, to formulate supported conclusions. Triangulation of information increased the reliability and potential transferability of findings, and increased the likelihood obtaining the same or similar results when researching with similar if not identical factors (Lincoln \& Guba, 1985; Merriam \& Tisdell, 2016). Furthermore, it was the goal of this researcher to utilize information gathered to expand knowledge related to how district goals translate into building goals, affect teacher instruction, and affect student learning through implementation of formative assessment using technology.

\section{Data Collection}

To utilize the program for this research, the Superintendent of Schools, by way of the DIT consented to the scope of this study by signing the gatekeeper consent form (see Appendix A). The DIT is composed of the district Superintendent and other leaders within the district whose responsibilities include instruction, professional development, facilities, technology, and human resources. Then, the proposal was submitted to the University of Missouri's Institutional Review Board (IRB). Once IRB approval was received (see Appendix B), the researcher began data collection for the proposed study. 
dissertation in practice, B. Jacobs

Consent was needed from teachers, instructional coaches and technology integration staff

(See Appendix C), as well as building and district administrators (see Appendix D) utilizing an informed letter of consent. Utilizing coding to identify participants (see Appendix $\mathrm{H}$ ), the researcher anonymously presented information and data to support emerging concepts and themes.

During the process of collecting data for this case study, several sources were utilized. These included interviews with building and district administrators, focus groups with mathematics teachers and district instructional support staff, analysis of district and building documents, and observation daily activities. In addition, this researcher conducted an in-depth examination of relevant literature related to how learning is impacted by feedback (Andersson \& Palm, 2017; Beatty \& Gerace, 2009; Black \& Wiliam, 2009; Wiliam, 2011), cooperative and collaborative learning experiences (Magaña \& Marzano, 2014; Marzano, 2006), and technology-enhanced instruction enhanced within mobile learning (Crompton, 2013; Hwang \& Chang, 2011; Puentedura, 2013; Reeves et. al., 2017; Romrell et. al., 2014; Xie, 2016).

Within qualitative research, researchers (Creswell, 2014; Merriam \& Tisdell, 2016; Yin, 2003) asserted it is necessary to take steps before collecting data that will increase validity, reliability and transferability of findings. According to Creswell (2014), "Validity and reliability of scores on instruments lead to meaningful interpretations of data" (p. 155). To accomplish this goal, this researcher chose to conduct a pilot, or field test: a method described by researchers (Creswell, 2014; Dikko, 2016, Merriam \& Tisdell, 2016) as a mini version of the research study used to increase the validity of the instruments used within the study. Researchers described face validity 
as a subjective analysis to determine whether the measurement makes sense, and is measuring the intended target (Nevo, 1985; Wheeler, Goolkasian, Baird, \& Darden, 1999). Further, Dikko (2016) describes content validity as ensuring "the instrument of measurement has tapped the concept it sets out to measure by including an adequate representation of items that operationalize the concept" (p. 521). The researcher utilized results from pilot testing to improve the data collection protocols, thus increasing face and content validity to improve accuracy when interpreting findings. An additional objective of pilot testing is to increase the internal validity, or credibility, of findings, thus creating a study that better captures reality and strengthens the argument in support of the findings (Merriam \& Tisdell, 2016).

\section{Focus Group Protocols}

Focus groups are a method to collect qualitative data through the opinions, statements, mannerisms, and group dynamics of participant groups (Creswell, 2014; Gill, Stewart. Treasure, \& Chadwick, 2008; Krueger \& Casey, 2014). The researcher conducted on-site, semi-structured, focus groups composed of mathematics teachers $(\mathrm{n}=$ 15) to obtain information regarding the implementation and effectiveness of technologybased formative assessment feedback on student learning (see Appendix B). Additionally, a focus group was conducted comprised of the district-level curriculum, technology, and instructional specialists $(n=6)$. The focus group protocols (see Appendix B) were developed as a series of questions guided by the research questions, and designed to provide information regarding the impact technology-based formative assessment feedback has on student learning and achievement in $7^{\text {th }}$ and $8^{\text {th }}$-grade mathematics. Once recordings were transcribed, members of each focus group were provided a transcript to 
verify the context and content of their statements. Researchers (Creswell, 2014; Lincoln \& Guba, 1985; Seidman, 2013) defined member checking as a practice utilized by researchers to validate the statements and opinions of members within a group or interview to increase trustworthiness and credibility of the report. Focus groups occurred on site or via video conference and lasted approximately 60 to 90 minutes.

Questions within the focus group protocols (see Appendix B) were designed to elicit information specific to the knowledge, abilities, application, and access to technology of the participants as related to formative assessment feedback within the teaching and learning cycle. The questions within this protocol focused on obtaining information relative to teachers knowledge of formative assessment feedback strategies (Black \& Wiliam 1998; Cowie \& Bell, 1999; Wiliam, 2011), skill set regarding embedding formative assessment and technology within their lesson design (Crompton, 2013; Hwang \& Chang, 2011; Puentedura, 2013; Reeves et. al., 2017; Romrell et. al., 2014; Xie, 2016) and perception of professional development in regard to both formative assessment and technology integration. This process allowed the researcher to develop focus group questions specifically designed to elicit responses relevant to the experiences and expertise of the participants as it related to the impact of technology tools and applications on formative assessment feedback.

After focus group questions were developed, a pilot study of the focus group protocol questions was conducted with a selection of $7^{\text {th }}$ and $8^{\text {th }}$-grade mathematics teachers $(n=2)$, and instructional specialists $(n=2)$ independent of those chosen to participate in the study. Researchers (Creswell, 2014; Fink, 2015; Krueger \& Casey, 2014; Siedman, 2013) stated pilot testing allows the researcher to adjust instrumentation 
based on the feedback obtained from participants of the pilot test, thus improving the questions and format of the data gathering process. Using the results and feedback from pilot testing of focus group protocols allowed the researcher to ensure questions address the desired content area and make adjustments to questions as needed before conducting the study, thus increasing the studies content validity (Creswell, 2014; Dikko, 2016; Fink, 2015; Merriam \& Tisdell, 2016). Ultimately, pilot testing increased content validity within focus group protocols, as the researcher was able to identify whether the questions elicit responses relevant to the topic of student learning related to formative assessment feedback and MLT. In addition, researchers outlined the utilization of pilot testing to adjust the wording and format of questions as a method of increasing the face validity of the protocols (Nevo, 1985; Wheeler, Goolkasian, Baird, \& Darden, 1999). As such, the researcher worked with participants to determine whether the questions were constructed appropriately, to identify areas of ambiguity, and to ensure questions were clearly understood (Creswell, 2014; Krueger \& Casey, 2014). Thus, adjustments to the protocols based on the feedback from the pilot study increased the content and face validity of the focus group protocols.

\section{Interview Protocol}

Researchers (Seidman, 2013; Schutz, 1967; Van Manen, 2015) stated the interview process should elicit the subjective experiences of participants resulting in a retrospective analysis of the meaning of lived experiences, thus revealing a particular point of view. The purpose of in-depth interviewing is to explore the experiences of people as a function of the meaning they associate with those lived experiences (Seidman, 2013). Seidman further highlighted the recognition and affirmation of the role 
of the interviewer as a skillful and intentional adaptation of the instrumentation. When interviewing building administrators, the researcher utilized a phenomenological approach to interviewing with an emphasis on technology-based formative assessment. As such, the researcher designed a series of open-ended, clarifying, probing and followup questions to "encourage participants to engage in that 'act of attention' that then allows them to consider the meaning of lived experience" (Seidman, 2013, p. 19). The researcher developed an interview protocol form (See Appendix B) to organize data collected in an accessible format for analysis and triangulation. The interview protocol contained eleven questions developed by the researcher, guided by the research questions, and conducted in a semi-structured format to identify the impact of technology-based formative assessment feedback on student learning. Interviews were scheduled to be approximately one hour in length.

Questions within the interview protocol (Appendix B) were designed to identify how formative assessment feedback and technology integration, singularly or in conjunction with one another, are incorporated within the district plan for teaching and learning. Further, questions within the designed interview protocol were intended to obtain the perceptions of participants related to the literature specific to formative assessment feedback and MLT to triangulate information gathered from other data collection methods (Seidman, 2013). Ultimately, the questions were designed to determine the specific perception of building and district level administrators regarding formative assessment feedback and technology integration as it relates to the district Comprehensive School Improvement Plan (CSIP). As stated by researchers (Marzano, 2014; Rutheford, 2013; Wiliam, 2011), meaningful feedback provided as a function of 
peer collaboration, self-reflection, or instructor review has a positive impact on student learning. A successful plan for professional development, according to Wiliam (2011), develops skills in eliciting evidence of learning to provide feedback, adjusting future lessons and learning outcomes, and allowing students to use feedback to improve performance.

After interview questions were developed, a pilot study of the interview protocol questions was conducted with a selection of building and district level administrators $(n=2)$ independent of those chosen to participate in the study. As previously stated, pilot testing allows researchers to increase the content validity of the instrumentation (Creswell, 2014; Seidman, 2013). The purpose of the pilot test was to increase content validity by analyzing whether questions elicit responses relevant to the desired content of the study (Fink, 2015; Siedman, 2013). A secondary purpose of the pilot test was to increase face validity, which was accomplished through discussion with participants as to whether the questions are clearly understood and identify areas of ambiguity within the questions or process (Creswell, 2014; Fink, 2015; Krueger \& Casey, 2014). In this case, the researcher used feedback from the participants to adjust and reformat questions within the interview protocol to insure questions were clear, as well as elicit responses relevant to the content of the study. The pilot test of interview protocols increased the content and face validity of the instrumentation and the validity of potential findings.

\section{Document Protocol}

Researchers described documents as an array of evidence in written, visual, digital and physical form to understand realities, communicate information, and produce insight to a conclusion of research (Flick, 2009; Merriam \& Tisdell, 2016). Public records 
within qualitative research are official and ongoing documents within an institution and manifest in a variety of forms (Creswell, 2014; Merriam \& Tisdell, 2016). The researcher created a protocol for document analysis (see Appendix C) to catalog information related to the topic within the District. Documents will be chosen specifically related to gathering information related to how technology is acquired, maintained, and utilized for instruction. Furthermore, document analysis allowed for an examination of curriculum guides, assessments, teacher development related to technology, and results of data collected by the district regarding technology tools and applications. By utilizing documents, the researcher decreased the chances of a biased study that could affect the validity of results (Creswell, 2014). Further, triangulating data with results from focus groups, interviews, and observations allowed the researcher to examine the impact of technology-based formative assessment tools on specific learner objectives (Yin, 2003).

\section{Observation Protocol}

Observations within a qualitative research study provide recorded data within a social setting, including but not limited to interactions, conversations, artifacts, events and behaviors (Marshall \& Rossman, 2014; Merriam \& Tisdell, 2015; Patton, 2014). In this case, observations allowed the researcher to participate in what Patton (2014) referred to as formal and planned opportunities, where an observer has an opportunity to see a phenomenon unfold in a semi-natural fashion. Patton described the benefit of personal contact as being able to observe and understand the "context within which people interact - for understanding context is essential to a holistic perspective" ( $p$. 332). Where interviews and documentation provide an interpretive analysis of a 
particular phenomenon, systematic observation provides a firsthand account of events, allowing triangulation of collected data incorporating senses other than sight that can validate results (Merriam \& Tisdell, 2015; Yin, 2003). The researcher developed an observation protocol (see Appendix D), and observed a meeting where teachers discussed how technology-based formative assessment feedback is incorporated within the learning cycle. The researcher participated in what researchers (Gold, 1958; Schensul \& LeCompte, 2012) described as a participant-as-observer role, building relationships with the group and participating in the activities while gathering data. The second observation setting was conducted on-site through a meeting conducted by the administrative leadership team. The researcher participated in an observer as participant role where gathering data is the primary focus of observation and building a relationship with participants will allow information to be transferred (Gold, 1958, Merriam \& Tisdell, 2015). The final observation was conducted at the district-level through participation in a meeting with district leaders of curriculum, testing, data analysis, and technology. The researcher continued in an observer as participant role to determine if the district CSIP addresses the implementation of technology-based formative assessment. The data collected in these observations allowed the researcher to form conclusions regarding outcomes of technology-based formative assessment.

\section{Data Analysis}

According to researchers (Creswell, 2014; Flick, Scott, \& Metzler, 2014), qualitative analysis allows the researcher to purposefully select sites or individuals to gather and analyze data that best fit the research questions designed to obtain useful information. Within the data collection process, a constant comparative method was 
utilized to allow for comparison of commonalities across the data (Merriam \& Tisdell, 2016). Specifically, data were gathered and analyzed in a manner where information could be coded and triangulated to identify common themes (Yin, 2003). Seidman (2013) contended creating a systematic process for filing transcripts, tracking participants, accurately labeling recordings, and securing information are necessary steps in managing information. The researcher examined data utilizing codes (see Appendix H). Transcripts and field notes were read to identify and code emerging themes in order to reduce the risk of a biased analysis (Seidman, 2013). To code the transcripts, each line was numbered consecutively to provide structure to the coding process. The researcher read all transcripts to associate responses from participants about the research questions. The researcher used a coded color system to match interview and focus group responses to corresponding research questions. Once responses are sorted, an analysis of responses from all participants was conducted to identify emerging themes. According to Tesch (2013), a researcher sorts through collected data to define categories and their properties, at which time a comparison between categories can be analyzed. Collecting and analyzing information while utilizing a variety of qualitative methods and data sources to identify the emerging theme is referred to as triangulation (Creswell, 2014; Fowler, 2004; Merriam \& Tisdell, 2016; Yin, 2003). Furthermore, Merriam and Tisdell (2016) suggested interviews and focus groups be recorded and transcribed by the researcher. The researcher provided a transcript to participants to complete the member checking process (Creswell, 2014; Seidman, 2013). Member checking was completed to account for misunderstood or potentially biased information throughout the process (Creswell, 2014). Member checking also allowed the participants to provide additional information 
that may not have been discussed within the focus group or interview process. (Creswell, 2014; Lincoln \& Guba, 1985; Seidman, 2013). In addition to recorded data, the researcher took field notes to document information not reflected on recorded focus groups or interviews, a method Krueger and Casey (2014) described to enhance information gathered.

\section{Limitations and Assumptions}

Limitations exist within all studies and considered by researchers before formulating conclusions or making recommendations (Creswell, 2014). Identifying the potential of internal and external threats aided the researcher when designing the study and increased the validity of findings (Creswell, 2014). It is imperative within qualitative research to check for accuracy, as well as use consistent methods when gathering and analyzing data (Creswell, 2014). Furthermore, increasing reliability within the findings by using valid and consistent methods increased the authenticity, credibility, and trustworthiness of conjectures (Creswell, 2014; Lincoln, Lynham, \& Guba, 2011).

Further, the researcher considered the steps the district has taken to increase access to devices, applications, and software, including measures to create an infrastructure conducive to technology infusion. Application of the information gathered within this study may prove useful to other schools and school districts, but will have to consider the variables within each setting individually (Creswell, 2014). Personal bias was another limitation of this study, as the researcher worked within the setting during the time of data collection (Creswell, 2014), creating a working knowledge that could inadvertently insert information into the findings not gathered by the designated instruments; i.e. focus groups, interviews, document analysis, and observation data. To 
account for potential limitations, bias, or validity of findings, this researcher utilized member checking and triangulation (Creswell, 2014, Yin, 2003).

\section{Design Controls}

To reduce bias within the study, the researcher purposefully used the following controls. Focus group and interview questions were open-ended questions, designed specifically to gather information from the perspective of the participants (Merriam \& Tisdell, 2016). Focus group and interview protocols had specific clarifying questions provided to all participants consistently. Member checking allowed the participants to clarify and potentially expand upon statements and thoughts made within each process, as well as increase the internal, content and face validity of instrumentation (Creswell, 2014; Merriam \& Tisdell, 2016; Seidman, 2013).

\section{Definitions of Key Terms}

The following defined key terms will provide a deeper understanding of the constructs and their facets.

Digital Natives: Students who have been using technology in their daily lives since birth (Martin, 2015), and therefore think and process differently than those who are adapting to the world with technology.

Educational technology applications: Programs designed for a specific purpose or task utilizing technology as the medium for accomplishing the task.

Educational technology tools: Technology hardware specifically designed to enhance the teaching or learning process through its physical presence.

Feedback: Information about reactions to a product, a person's performance of a task, etc. which is used as a basis for improvement of instruction (Wiliam, 2011) 
dissertation in practice, B. Jacobs

Formative Assessment: An assessment where "evidence about student achievement is elicited, interpreted, and used by teachers, learners, or their peers to make decisions about the next steps instruction that are likely to be better...than the decisions they would have made in the absence of that evidence" (Wiliam, 2011, p. 43).

Missouri Learning Standards- In 2014 Missouri legislators passed House Bill 1490, mandating the development of the Missouri Learning Expectations. In April of 2016, these Missouri Learning Expectations were adopted by the State Board of Education. Groups of Missouri educators from across the state collaborated to create the documents necessary to support the implementation of these expectations. (Department of Elementary and Secondary Education, 2019)

Traditional - what students should know and be able to do

Technology - the International Society for Technology Education (2016) defines seven standards for students within educational technology-empowered learning, digital citizenship, knowledge construction, computational thinking, creative communication, and global collaboration.

$\mathbf{2 1}^{\text {st }}$ Century Skills: Skills that drive competitiveness and innovation (Van Laar, Van Deursen, Van Dijk, \& De Haan, 2017) thus the need to make sure students "obtain strong learning skills in information gathering, communication, critical thinking and problem solving" (O’Sullivan \& Dallas, 2017, p. 3)

\section{Significance of the Study}

Although researchers have provided evidence to support the effectiveness of collaborative and cooperative learning (Magaña \& Marzano, 2014; Marzano, 2006) and embedding formative assessment within instruction (Black \& Wiliam, 2009; Wiliam, 
dissertation in practice, B. Jacobs

2011), researchers (Chu, 2013; Hwang \& Chang, 2011; Sung, Chang \& Liu, 2016)

further identified the need for research related to the impact of mobile devices on student learning. Crompton and Burke (2017) asserted the need to provide practitioners with a guide for utilizing and effectively implement mobile learning, reducing the emphasis on student-centered initiatives and in influencing student learning through instructional design.

Despite current research on the impact of various technologies, formative assessment, and knowledge construction with increased access to mobile devices, the questions remain; what role does technology play within the teaching and learning process specific to formative assessment and what does that mean for instructional practice in teaching and leadership roles? Researching this problem of practice provided specific and usable information to administrators and teachers as they create and plan lessons integrating technology as a means to assess student comprehension of standards. The results of this study provided sufficient data to guide the creation and execution of a professional development plan enhanced by technology-based formative assessment. Specifically, the researcher provided an analysis of qualitative data to determine the connection between increasing opportunities for technology-based formative assessment and an increase in student learning. Data collected through focus groups, interviews, document review, and observations was used to support conclusions, make recommendations to District, and provide recommendations to similar districts where findings may be transferrable (Creswell, 2014; Merriam \& Tisdell, 2016; Siedman, 2013). 
dissertation in practice, B. Jacobs

In summary, this study added to the literature on MLT specific to the perceived effects of technology on formative assessment feedback concerning student learning. Data were gathered and analyzed through a basic qualitative approach, utilizing a case study to gather information analyzed through a social constructivist lens. The purpose was to obtain sufficient data based on the constructed meaning of multiple sources to provide evidence supporting a conclusion. Furthermore, analysis of this problem provided evidence to support, or refute, claims that increasing the frequency of formative assessment feedback utilizing technological tools and applications positively impacts student learning. Such results helped to determine whether practitioners consider increasing formative assessment as an impactful variable as they develop action plans related to student achievement.

\section{Summary}

Researchers have made a strong argument in support of formative assessment feedback (Black \& Wiliam 1998, 2009; Wiliam, 2011) and how the increase of formative assessment feedback within instruction ultimately impacts learning. Other researchers (Cho, 2005; Magaña \& Marzano, 2014; Marzano, 2006) examined concepts of learning theory, feedback, and cooperative learning regarding the impact on student achievement data. Furthermore, researchers examined the effect of technological tools on student achievement, but failed to delineate specific variables beyond technology to student ratio or increasing classroom use of tools as differentiated factors (Cheung \& Slavin, 2013; Eyyam \& Yaratan, 2014; Grant, Tamim, Brown, Sweeney, Ferguson, \& Jones, 2015; Lei \& Zhoa, 2007). As such, there are many facets to explore to examine the impact of technological tools. MLT has elevated technology beyond a mere substitution of 
pedagogy, and thus expanded the opportunity to examine the impact of instant, real-time, and unbounded learning experiences. Results of this study provided insight and evidence with concern to how regular and intentional use of mobile, technology-based, formative assessment impacts student achievement. 


\section{SECTION TWO}

PRACTICTIONER SETTING FOR THE STUDY 


\section{Introduction}

This section of the dissertation-in-practice proposal includes a discussion of how educational organizations have implemented technology-based instruction within teaching and learning. District has incorporated and implemented technology within its organization somewhat autonomously; however, it is imperative to this study to discuss the history of how infused technology has impacted the culture of education throughout the $20^{\text {th }}$ and early $21^{\text {st }}$ centuries. Throughout this timeframe, the specifics, the when and the how, of implementation have varied between school districts. The development of technology standards within education have affected the way school districts approach instructional design to impact student learning. This section also includes an analysis of

the organization as it relates to the research study. Next, the discussion focused on leadership analysis and finally, the implications for research in the practitioner setting explored.

\section{History of the Organization}

The setting used to answer the research questions were three middle schools within the District. The District was founded in 1949, and has a long history of being recognized with distinction based on the academic achievements of its students. The District serves a suburban portion of Missouri within 50 miles of the Kansas City area, encompassing part or all of eight cities in close proximity to one another. The District currently has an approximate population of 100,000 patrons, of which enrollment for 2017-2018 is estimated at 18,157 students served by 18 elementary schools (K-6), three middle schools (7-8), three high schools (9-12), an alternative secondary school (7-12), a secondary technology academy (11-12), an early education center (PK), and a special 
education day-treatment center (K-12). Since 1990, enrollment has shown an average increase of approximately 335 students per year district-wide, creating the need for two additional sites for middle and high school. The district uses the same boundaries for each middle school and high school pairing, where the entire population from each middle school feeds directly into the high school in the corresponding boundary.

The district has a reputation for being innovative and on the cutting edge with the programs and services offered to students. Currently, the district is within its sixth version of its Comprehensive School Improvement Plan (CSIP). The current format for school improvement began in 1990 as a collaborative approach, bringing community members within the district and staff members together every five years to identify specific strategies to meet state and district objectives. In this plan, the district addressed technology resources and technology-enhanced instruction within one of its focus areas: Support and Instructional Resources. Specifically, the district technology department used standards developed by International Society for Technology in Education (ISTE) to identify specific skills and knowledge students must learn to demonstrate competency. These standards have evolved within the ISTE organization over 40 years, incorporating changes in technology access, tools, and applications connecting educators in a collaborative approach to teaching with technology. To discuss the history of technology within the District organization, this researcher analyzed the development of technological initiatives, specifically, evaluating the impact of technology standards through the infusion of digital devices and applications.

The original version of the ISTE standards was published in 1998, and was known as the National Educational Technology Standards (NETS). The purpose at the time was 
to focus on the skills students would need to be prepared for post-secondary life, and has since been revised in 2016. In addition, ISTE developed standards for teachers in 2008, revised in 2017 as the ISTE standards for educators, for administrators in 2009 and revised in 2017, as well as ISTE standards for coaches and computer science educators, both released in 2011. As the ISTE standards have been introduced and incorporated within the K-12 educational setting, researchers (Martin, 2015; Wetzel, Foulger, \& Williams, 2008) asserted school districts, as well as higher education institutions, must consider providing experiences and training with the intention of supporting successful technology integration. From the ISTE standards, a variety of frameworks have been developed by educators in District and in districts nationwide, providing technologybased approaches that enhance the teaching and learning process.

As previously stated, the District is composed of three middle schools, chosen because they house $7^{\text {th }}$ and $8^{\text {th }}$ grade students and data collected provided information directly related to the study. Each of the middle schools were constructed as a function of population growth within district boundaries. The first of the three middle schools, Site A, opened in 1971. As described by the Department of Elementary and Secondary Education (2018), this middle school comprised of approximately 850 students in $7^{\text {th }}$ and $8^{\text {th }}$-grade residing in the middle, southern and eastern quadrants of the district boundary. The population of Site A is $79 \%$ White, $10 \%$ Black, $5 \%$ Hispanic, $4 \%$ are two or more races, $2 \%$ Asian, and less than 1\% American Indian. Approximately $27 \%$ of the population qualifies as low-income based on eligibility requirements for free or reduced lunch. The teachers within this site hold at least a bachelor's degree and certification in one or more educational areas, and have teaching experience that ranges from one to 29 
years teaching. Of the 68 teachers within Site A, 59 hold at least a master's level degree or above, including one who holds an educational specialist.

The second middle school, Site B, is comprised of approximately 920 students in $7^{\text {th }}$ and $8^{\text {th }}$-grade who reside across the northern boundary of the district boundary. The Department of Elementary and Secondary Education (2018) described the population of Site B is $71 \%$ White, $16 \%$ Black, $7 \%$ Hispanic, $4 \%$ are two or more races, $2 \%$ Asian, and $1 \%$ American Indian. Approximately $25 \%$ of the population qualifies as low-income based on eligibility for free or reduced lunch. The teachers within this site hold at least a bachelor's degree and certification in one or more educational areas, and have teaching experience that ranges from one to 28 years teaching in the district. Of the 63 teachers within Site B, 55 hold at least a master's level degree or above, including four who hold an educational specialist, and one who holds an educational doctorate.

The third middle school, Site C, opened in 2,000 with an original capacity of 600 students, which was later expanded in 2004. Site C is currently comprised of approximately 1,100 students in $7^{\text {th }}$ and $8^{\text {th }}$-grade residing in the southern and western quadrants of the district boundary. The population of Site $\mathrm{C}$ is $80 \%$ white, $11 \%$ Black, 4\% Hispanic, $4 \%$ are two or more races, $2 \%$ Asian, and less than $1 \%$ American Indian. Approximately $11 \%$ of the population qualifies as low-income based on eligibility for free or reduced lunch (Department of Elementary and Secondary Education, 2018). The teachers within this site hold at least a bachelor's degree and certification in one or more educational areas, and have teaching experience that ranges from one to 30 years teaching in the district. Of the 69 teachers within Site C, 57 hold at least a master's level degree or above, including five who hold an educational specialist. 
dissertation in practice, B. Jacobs

Each setting contains a representative group of students comparable to that of the district. Subgroups within each site are comparably represented, with the exception being a variance when considering the socio-economic representation at Site C (See Table 1). Although the percentage of the population receiving free or reduced lunch is less than the other two sites, the identified percentage is still representative as it relates to the district percentage of nineteen. As such, the three sites, composed of highly qualified teachers located within a district so highly regarded for educational accomplishment, provides a rich environment for data related to this study.

Table 1

Description of Organization Structure of the Participants

\begin{tabular}{|c|c|c|c|c|c|c|c|}
\hline \multirow[b]{2}{*}{ School } & \multicolumn{2}{|c|}{ Student } & \multicolumn{3}{|c|}{ Teacher } & \multicolumn{2}{|c|}{ Principal } \\
\hline & Enrollment & $\begin{array}{c}\text { F/R } \\
\text { Lunch }\end{array}$ & Total & $\begin{array}{l}\text { Avg } \\
\text { Exp }\end{array}$ & $\begin{array}{c}\text { Advanced } \\
\text { Degrees }\end{array}$ & $\begin{array}{l}\text { Avg } \\
\text { Yrs at } \\
\text { Site }\end{array}$ & $\begin{array}{l}\text { Highest } \\
\text { Degree }\end{array}$ \\
\hline A & 841 & $27 \%$ & 68 & 14 & $86.8 \%$ & 14 & Doctorate \\
\hline B & 934 & $25 \%$ & 63 & 12 & $87.3 \%$ & 3 & $\begin{array}{l}\text { Educational } \\
\text { Specialist }\end{array}$ \\
\hline C & 1134 & $11 \%$ & 69 & 14 & $82.6 \%$ & 7 & Doctorate \\
\hline
\end{tabular}

Note: $\mathrm{N}=\mathbf{5}$ interview participants; 3 building level principals; $\mathrm{N}=4$ focus groups; participants 1-15 were teachers from 3 school sites, participants 16-21 were district level instructional level support staff

\section{Organizational Analysis}

Examined through the structural frame, the District possesses characteristics of what Bolman and Deal (2013) referred as a machine bureaucracy - where "managers and standardized procedures govern day-to-day operations" (p. 77). Before the adoption of 
the district initiative providing one device per student, teachers in District were using devices for instructional purposes as a means to supplement learning activities with limited, district-supported devices provided at each site. As such, teachers established classroom procedures for checkout, supervision, communication, and citizenship to manage or deter potential disruptions. Post implementation of the initiative, district and building staff considered obstacles to implementation affecting the day-to-day operation of the building with the additional goal of modifying instruction across all disciplines. Items for consideration included, but were not limited to, access to electronic devices beyond the school day, maintenance and care of devices, and procedures for lost, stolen, or damaged items, and thus, how each affected classroom instructional time. Understanding the current community and structure, developing new goals, designing a new structure incorporating the voices and viewpoints of all stakeholders, and studying results are key principles of a successful organizational change (Bolman \& Deal, 2013; Stone, 2012).

As the District developed strategies for incorporating technology as an instructional tool, implementation sites developed a process for allocating responsibilities for decision making in addition to a method allowing for all voices to be heard (Bolman \& Deal, 2013). According to Mintzberg (as cited in Bolman \& Deal, 2013, p. 75), an organization is a structure consisting of five parts: (1) operating core, (2) technostructure, (3) support staff, (4) middle line managers, and (5) strategic apex. Theories indicated the core premises of the structural frame are to identify clear goals, roles and relationships and coordinate efforts to meet performance standards (Bolman \& Deal, 2013). Utilizing Mintzberg's (2005) model, this researcher identified key players and 
their role within the organization. The strategic apex included the Board of Education (BOE), superintendent, associate and assistant superintendents, and district level executive directors. Middle-line managers and members of the techno-structure work as teams to analyze and make recommendations to the district. Middle-line managers included the building administration and curriculum specialists who monitor the scope and sequence of curriculum as well as the allocation of resources. The techno-structure consists of technology and instructional specialists who coordinate the implementation of specific instructional design to promote achievement of learning standards. Situationally, staff members at the district and building level can move between groups - for example, the Director of Technology could serve in the strategic apex and as a member of the techno-structure.

Teachers were situationally transient in this initiative. They serve in the operating core when addressing colleagues, staff members, or administrators. Moreover, they serve as middle managers when interacting with students and external constituents. Some teachers serve within the techno-structure by providing technology support, professional development, research, and implementation strategies. Teachers who possess technology expertise serve within the techno-structure and middle line management. Students themselves were the operating core as they are ultimately responsible for carrying out the initiative, but lack autonomy to make decisions (Mintzberg, 2005).

The examination of this organization from the political frame (Bolman \& Deal, 2013, Manning, 2017; Stone, 2012) allowed for analysis of how members of the organization react concerning the diverse interests of its constituents. The key actors in this dilemma were the parents, students, district-level administrators, building-level 
administrators, non-instructional staff, and instructional staff. Charged with allocating resources to maintain and support learning and instruction to meet specific goals of student learning and achievement were district and building level administrators and directors (Bolman \& Deal, 2013). Within the structure, decisions must be made regarding initiatives that directly impact professional development and training for teachers. Political considerations included, but are not limited to, how to establish normative behavior, standards for excellence, and communication of core values (Manning, 2017), as well as determining the autonomy level for teachers regarding implementation of technology-based instruction and formative assessment strategies. Further, allocated are district resources to provide communication and training to students, adjust curricular scope and sequence, and increase the availability of technical support. Building-level administrators served as front-line implementers for parents, students, and teachers. Specifically, assisting groups within the organization will take place to adjust and adapt the mindset of incorporating technology as a necessary component of student learning.

Moreover, each team decided how to allocate financial and human resources to maximize the efficiency of building operations to support student learning. Teachers exhibited a variety of experiences related to technology, instruction, and assessment that provided a valuable resource to the building. Politically, teachers in support of an initiative will result in a successful transformation of the building culture, where a lack of support, or proper training, can derail the initiative (Bolman \& Deal, 2013; Manning, 2017). As expectations related to their job description change, teachers and staff members will respond to increased responsibility by vying for potentially scarce resources. 
dissertation in practice, B. Jacobs

Bolman and Deal $(2010,2013)$ asserted there are key skills that are essential when strategically addressing these dynamics within the political frame. These skills include: (a) agenda setting, (b) mapping the political terrain, (c) networking and building coalitions, and (d) bargaining and negotiating. To set the agenda for instructional change as it relates to the technology-based formative assessment, there are a variety of perspectives on how goals for implementation should be developed. Building-level decision makers value increasing overall student achievement through differentiating instructional pedagogy of teachers, increasing opportunities for collaboration between students and staff members, and improving instructional effectiveness. Mapping the terrain within the organization is a consideration of whose power will complement the program (Bolman \& Deal, 2013; Pfeffer, 1992). Once power players have been identified, leaders must build alliances through creating incentives that strengthen validity of the initiative within the organization (Manning, 2017; Stone, 2012). Networking and negotiating are dependent on one another, as it is a likely function of change that each will have to make concessions (Bolman \& Deal, 2010; Stone, 2012). Collaborating with key players, such as veteran teachers and department heads, will increase the likelihood that groups with opposing viewpoints will dialogue and eventually compromise. Aligning with teachers who possess expertise in technology instruction will increase opportunities for them to lead professional development.

In the educational environment, power over the functions within the environment primarily fall with building administrators (authorities) and teachers (partisans) (Bolman \& Deal, 2013). As authorities, administrators control aspects of working environments and make decisions that subordinates must follow. As a result, partisans lack connection 
to change initiatives and are compelled to promote strategies that originate from the bottom-up. This creates conflict where both groups perform with a sense of being mutually exclusive. However, there is an interdependent relationship that exists between authorities and partisans as they rely on each other for assistance and support (Bolman \& Deal, 2013). The symbiotic ecosystem that exists will suddenly introduce a new element that can change the landscape that has short and long-term effects, similar to a genetic alteration of the DNA of an organism (Bolman \& Deal, 2013).

\section{Leadership Analysis}

The definition of leadership has changed from the early $20^{\text {th }}$ century; the assertion of power used to control and manage (Hemphill, 1949; Moore, 1927), to the $21^{\text {st }}$ century; an inclusive, transformational model of using influence to achieve the desired results separating leadership and management (Burns, 1978; Kotter, 1990; Northouse, 2015). Although researchers (Judge, Bono, Ilies, \& Gerhardt, 2002; Northouse, 2015; Zaccaro, 2007) conveyed traits which can assist leaders in making a quality influence, they also asserted process-based leadership as a series of intentional interactions utilized to invoke the desired results between leaders and followers in conjunction with the traits, skills and abilities (Dansereau, Graen, \& Haga, 1975; Northouse, 2015; Tyler \& Creemer, 2005). As such, researchers (Hussain, Talib, \& Shah, 2014; Northouse, 2015; Storey, Hartley, \& Denis, 2016) asserted leadership has evolved into a process of decisions and influences, involving a mutual effect between groups of individuals, with distinct attention to common goals and developing human capital as a part of an effective design. In addition, such leadership is a function of commitment, where clarity and buy-in creates teams that establish clear definitions for goals, a common method for attaining success 
within those goals, and a unified communication plan for confronting conflict (Levi, 2014; Lencioni, 2002; Storey et. al., 2016).

As a result of leadership being viewed as a process-based partnership between leaders and followers, different approaches to leadership have emerged based on the characteristics, skills, strategies, and actions a leader utilizes to motivate followers to achieve desired results. In conjunction, the impact of the leader depends on the objectives and methods identified within the organization (Storey et. al., 2016). For example, researchers (Dionne et. al., 2014; McCleskey, 2014; Thompson \& Glaso, 2015) indicated situational leadership prescribes a variety of leadership actions depending on the objective, skills, traits, and readiness of those within the organization, and the maximum capacity for results. Where situational leadership focuses on the leader adjusting to the needs of the situation, leader-member exchange (LMX) theory encourages building trust through high-quality interactions between each follower, purposefully engaging in the process of meeting organizational goals (Dionne et. al., 2014; Erdogen \& Bauer, 2014; Liden, Sparrowe, \& Wayne, 1997). According to researchers (Northouse, 2015; Wayne, Shore, Bommer, \& Tetrick, 2002; Wang, Law, Hackett, Wang, \& Chen, 2005), LMX theory challenged the notion that leaders treat followers in a collective way by emphasizing the effect of utilizing high-quality relationships to advance the goals of the leader, followers, and the organization. Researchers (Hernandez \& McKenzie, 2010; Johnson, 2006; Mikelic, Lipicnik \& Tekavcic, 2010) described factors leaders should consider when developing their organization, such as individualization, equity, and inclusion. As such, Bennis and Nanus (1985) asserted transformational leaders have a vision, create a set of shared 
values within people, establish a culture of trust, and utilize their strengths to create momentum and confidence within the organization.

Where the leaders of the district exhibited a variety of individual leadership approaches, the leadership style evident within District was situational. The needs of teachers, students, staff members, schools and departments varied in nature, creating the necessity of separate approaches to the organizations within the organization. As such, the District utilizes what DuFour and Eaker (1998) defined as a Professional Learning Community (PLC) to create a partnership between district leadership, staff, community members, and students allowing for decisions made considering multiple interests and perspectives. Situational leadership demands a leader be able to evaluate the current environment to determine the level and type of support needed to maximize efficiency (Storey et. al., 2016; Thompson \& Glaso, 2015). Northouse (2015) characterized situational leadership into levels, in which the type of support provided by leaders is dependent on the development level of the follower. Blanchard, Zigarmi, and Zigarmi (1985) provided a model of situational leadership that included directing, coaching, supporting, or delegating within leadership in response to the competence and motivation level of followers. Furthermore, considering equity, or accounting for dimensions of fairness and inclusion as it relates to the needs of stakeholders, is essential when making decisions within situational leadership (Simon, Malgorzata, \& Beatriz, 2007). As such, the District created a culture of leadership based on the needs of the constituents and provided for autonomy within individual sites to make decisions unique to their goals within the CSIP. 
dissertation in practice, B. Jacobs

Cox (2015) asserted a leader should challenge the assumptions of staff members in order to connect a community of learning, as well as continuously revisit how learning outcomes connect to the values of staff members. Researchers (Burns, 1978; Northouse, 2015; Yammarino \& Dubinsky, 1994) have described a transformational leader as one who increases motivation and morality of leaders and their followers through addressing the motives and needs of followers as a symbiotic relationship of growth.

Transformational leaders influence the ethics and morality of followers, creating a transformation those researchers (Avolio \& Bass, 1995; Zhu, Avolio, Riggio, \& Sosik, 2011) asserted increases the likelihood of moral actions and decisions by followers. As transformational leadership has evolved, Bass (1985) identified three dimensions of transformational leadership: charisma, individualized consideration, and intellectual stimulation. These dimensions explore levels of modeling behavior, inspire creativity and risk taking, create a culture of high expectations, and individualize support by listening to the needs of stakeholders in order to maximize the productivity of followers. As a result, transformational leadership yields results far beyond the expected or desired outcomes (Bass \& Riggio, 2006; Northouse, 2015; Yammarino \& Dubinsky, 1994). The Superintendent of Schools in the District has exhibited qualities such as these throughout his tenure. The appeal the Superintendent makes to all individuals within the district is to examine trends within district data to make the best, most equitable, research-based decisions for programming is evident. The Superintendent focused on building trust within his stakeholders, held staff and students to a high standard, and relied on the decision makers within the PLC to use the district mission and vision as a compass for all action. Researchers concluded transformational leadership has a positive impact on 
dissertation in practice, B. Jacobs

organizations in that leaders encourage employees to transcend their own interests (Bass \& Avolio, 1990), increase performance and company profit (Rowold \& Heinitz, 2007), and find a positive connection to job satisfaction and performance (Nemanich \& Keller, 2007). Furthermore, Carrejo, Cortez, and Reinhart (2010) asserted that the effectiveness of an organization, when considering the academic success of students, depends on the stakeholders viewing themselves as accountable leaders within the process of determining what and how students should learn. The responsibility of the leader, in the transformational sense, is to appeal to the emotions of followers in order to encourage leadership, thus transferring ownership of the objectives from the organization to the followers.

\section{Implications for Research in the Practitioner Setting}

The goal of this study was to provide a platform as a means to determine the impact of technological tools and applications on student learning. Specifically, this study provided information to practitioners on how technological tools and applications impacted the formative assessment feedback utilized to guide and change learning experiences for students. A vast amount of literature exists to support the positive effect of increasing formative assessment feedback to modify learning experiences (Andersson \& Palm, 2017; Beatty \& Gerace, 2009; Black \& Wiliam, 2009; Chappuis, 2015; Cowie \& Bell, 1999; Czaja et. al., 2006; Marzano, 2006; Nicol \& Macfarlane-Dick, 2006; Rusthon, 2005; Sadler, 2004; Wiliam, 2011). While an assumption exists which supports technological tools as an avenue to enhance formative assessment frequency and strategies, research was lacking to support this assertion 
(Wiliam, 2011). Research on the effects of increasing technology access within the learning process is broad in nature. Researchers have generally focused on supporting the infusion of technology as a function of accessing knowledge through a different medium (substitution), collaboration (augmentation), and meeting technology education standards (Anderson \& Dron, 2011; Andersson \& Palm, 2017; Ashfari, Bakar, Luan, Samah, \& Fooi, 2009; Alam, 2015; Barry, 2013; Burns et. al., 2010; Faber et. al., 2017; Grant et. al., 2015; Harasim, 2012; ISTE, 2016, 2018; Kposowa, 2013; Offer \& Bos, 2009; Pachler et. al., 2010). District, with the incorporation of technology based initiatives, utilized such research and the impact of increasing technological access to students within the district to increase student learning. This study was intended to influence district decisions specifically providing professional development to staff regarding the benefit of student learning by utilizing technological tools within formative assessment feedback.

\section{Summary}

The second section of this study introduced the school district and provided a foundation of how technology initiatives were incorporated within the District CSIP in conjunction with the national standards outlined by ISTE. The researcher provided an analysis of the organization from a structural and political frame, and thus a share the context on how decisions and initiatives are implemented, clarify the key stakeholders of decision-making, and provide an outline of the overlapping nature of roles within the district specific to the topic (Bolman \& Deal, 2010, 2013; Manning, 2017; Stone, 2012). The researcher provided evidence by citing relevant scholarship on organizational analysis. In conjunction, the researcher provided a scholarly analysis of leadership 
dissertation in practice, B. Jacobs

examining how the District operates as a unit, and how individual leaders incorporate their leadership style within the vision of the district. Finally, the researcher discussed the relevance of the current study and the implications of research within the setting. 
SECTION THREE 


\section{Introduction}

When properly used, formative assessment, according to researchers (Black \& Wiliam, 2009; Dunn \& Mulvonen, 2009; Nicol \& Macfarlane-Dick, 2006; Wiliam, 2011), is a highly effective and impactful way to obtain results specific to student learning. This researcher examined the impact of technology devices and applications on learning through formative assessment strategies specific to providing formative assessment feedback. This researcher examined several theoretical frameworks within learning theory such as behaviorism, cognitivism, connectivism, and mobile learning to determine whether learning is impacted by technology integration. The SAMR model, utilized to define objectives within technology integration as related to instructional design and student learning, was used to evaluate each construct (Jude et. al., 2014; Puentedura, 2013). Specifically, this exploration outlined factors within learning theory and examine whether technology-based formative assessment enhances, depletes, or is a negligible factor concerning student learning. As such, this scholarly review will analyze technology-based formative assessment and learning theory to determine if the effect of technological tools on formative assessment feedback is pronounced.

\section{Learning Theory as a Theoretical Framework}

Learning theory is a theoretical framework often used as a means to understand the way information is absorbed, processed, and retained within teaching and learning (Luis \& D'Cunha, 2014; MacCallum \& Parsons, 2016). According to researchers (MacCallum \& Parsons, 2016; Siemens, 2014), traditional learning theories are often categorized within the framework of behaviorism: learning as a function of changed behaviors; cognitivism: a commitment of processes to short, and long-term memory; and 
constructivism: the creation of knowledge from experiences. Research on the role of technology has lead researchers to explore connectivism: the process of learning in multiple environments where the foundation of information is in a constant state of fluctuation (Conradie, 2014; Dunaway, 2011; Siemens, 2014). In addition, mobile learning theory is the concept of utilizing mobile devices to provide a portable and personalized medium for: 1) gathering data, 2) communicating and connecting through an interface, 3) encouraging active learning, and 4) utilizing immediate feedback to inform instruction (MacCallum \& Parsons, 2016; Ozan \& Kesim, 2011; Parsons, Wishart, \& Thomas, 2016; Romrell et. al., 2014). Researchers (Harasim, 2012; MacCallum \& Parsons, 2016; Parsons et. al., 2016) debated whether technology in and of itself should warrant new learning theories. However, theories like connectivism (Kizito, 2016; Ozan \& Kesim, 2011; Siemens, 2014) and mobile learning theory (Ozan \& Kesim, 2011; Park, 2011; Parsons et. al., 2016) were the result of studies designed to identify how technology integration impacts learning. Furthermore, the SAMR (Substitution, Augmentation, Modification, Redefinition) evaluation model evolved as a way to analyze the impact of technology on teaching and learning (Jude et. al., 2014; Puentedura, 2013; Romrell et. al., 2014).

Researchers (Black \& Wiliam, 2009; Cowie \& Bell, 1999; Dunn \& Mulvonen, 2009; Wiliam, 2011) defined formative assessment as the process of gathering and utilizing data to inform and improve instruction to achieve results. Formative assessment feedback includes opportunities for clarification of goals, self-reflection and assessment, and collaboration, thus allowing teachers and students to identify and properly perceive gaps in learning, interpret the results, and make adjustments within learning activities 
(Biggs, 1998; Nicol \& Macfarlane-Dick, 2006; Rushton, 2005; Sadler, 1998; Vaughn et. al., 2013; Wiliam, 2011). Similarly, Nicol and Macfarlane-Dick (2006) and Wiliam (2011) proposed incorporating embedded formative assessment as a means to share teaching practices and provide feedback to students, both of which have a positive impact on student achievement. Infusing technology in classrooms where teachers are trained to enhance feedback and modify instruction based on information gained could be further studied (Vaughn et. al., 2013; Wiliam, 2011). Other theories, such as TechnologyEnhanced Formative Assessment (TEFA) and Formative Assessment-based Mobile Learning (FAML) emphasize the impact of technology on learning with mobile technology utilizing formative assessment strategies (Burns et. al., 2010; Lee et. al., 2012; Hwang \& Chang, 2011). Consequently, this researcher examined the aforementioned learning theories to determine the significance of technology-based tools on formative assessment and the impact on learning.

\section{SAMR Model}

As educational technology has developed, resources have been invested toward the infusion of devices and technology-based initiatives (Bebell, Russell, \& O'Dwyer, 2004). As such, examining the effects of technology on teaching and student learning created the need to explore the context in which technology usage is defined. For example, technology-based formative assessment has changed within pedagogy as technology devices and applications have evolved from Classroom Response Systems (CRS) to web-based assessment and feedback mechanisms (Beatty \& Gerace, 2009;

Burns et. al., 2010; Hwang \& Chang, 2011). In response, researchers (Jude et. al., 2014; Olmanson et. al., 2015; Puentedura, 2013; Romrell et. al., 2014) used the SAMR model 
to categorize technologies and its impact on student learning as compared to learning without such technological tools. As developed by Puentedura (2013), the SAMR model depicts four levels of technology integration (substitution, augmentation, modification, and redefinition) as a construct for educators to evaluate the effectiveness of learning activities specific to technology. Therefore, analyzing the impact of technology on learning necessitates a brief discussion of the model to determine whether traditional learning theory applies, or if technology is the catalyst to learning.

Within the substitution level of SAMR, researchers sited examples of physical substitutions that provide a different medium for instruction without a functional change (Hamilton, Rosenberg, \& Akcaoglu, 2016; Jude et. al., 2014; Puentedura, 2013; Romrell et. al., 2014). Examples may include using computers in place of traditional typewriters for document creation or word processing, using calculators to perform computational functions, or projecting computer images replacing traditional strategies of and presenting visual aid. In addition, substitution can be accomplished beyond the increase of technology tools to include a substitution for how information is obtained and exchanged (Puentedura, 2013; Romrell et. al., 2014). Researchers (Jacobs-Isreal \& Moorefield-Lang, 2013; Kihoza, Zlotnikova, Bada, \& Kalegele, 2016) described substituting for traditional methods for research, such as books or magazines, by providing access to online reading materials available using tablets, IPads, or Kindles. Additional examples of substitution include taking and storing notes in an online venue with web-based applications, using digital cameras and projectors to present projects, or citing online informational resources within research projects (Hamilton, Rosenberg, \& Akcaoglu, 2016; Jacobs-Isreal \& Moorefield-Lang, 2013). 
dissertation in practice, B. Jacobs

While substitution provides an opportunity for instructors to replace traditional techniques and strategies, researchers (Jacobs-Isreal \& Moorefield-Lang, 2013; Puentedura, 2013; Romrell et. al., 2014) asserted the augmentation of learning through technology enhances activities through increased functionality, such as when a computer offers spell check, cut and paste, and printing options. CRS's are an example of how technology substitutes and augments the teaching and learning process by allowing teachers to gather data from learners and use the data to adjust instruction (Beatty \& Gerace, 2009; Siau, Sheng, \& Nah, 2006). Hamilton, Rosenberg, and Akcaoglu (2016) provided examples such as a tablet providing increased functionality to a learning environment, allowing a student to access, read and listen to text independently. As such, substitution and augmentation have transformed classroom learning activities to incorporate the skills and abilities teachers and students have developed with the development and accessibility of technology, allowing for the same, or similar, learning outcomes with more efficient, technology-based, activities (Romrell et. al., 2014).

Modification, according to researchers (Hudson, 2014; Jude et. al., 2014; Puentedura, 2013; Romrell et. al., 2014), is characterized by the significant redesign of instruction elevating traditional learning experiences. Usually accomplished in concurrence with redefinition is modification, thus creating activities that could not otherwise be accomplished without integrating technology (Jude et. al., 2014; Puentedura, 2013; Romrell et. al., 2014). Examples of modification with technology integration may include utilizing computer simulations rather than diagrams to demonstrate a topic (Hamilton, Rosenberg, \& Akcaoglu, 2016), using a google document or e-mail to share, collaborate or peer review a report (Kihoza, Zlotnikova, Bada, \& 
dissertation in practice, B. Jacobs

Kalegele, 2016), or the use of the internet to complete a project (Jude et. al. 2014). Once teaching and learning is transformed into an experience dependent on technology to create a previously inconceivable or attainable task, the learning activity meets the standard of redefinition (Jude et. al., 2014; Kihoza, Zlotnikova, Bada, \& Kalegele, 2016; Puentedura, 2013). Some examples of redefined learning experiences may include video creation, inquiry-based learning experiences, teaching and learning mathematics lessons independently, or assessing students learning using technology (Jacobs-Isreal \& Moorefield-Lang, 2013; Kihoza, Zlotnikova, Bada, \& Kalegele, 2016).

The impact of technology on instruction and learning, as detailed by the SAMR model of evaluation, allowed this researcher to examine the constructs of each learning theory. According to Hudson (2014), "Substitution and Augmentation - represent technology that are simply enhancements to existing non-digital resources.... Modification and Redefinition - describe when a technology or application is truly transformative" (p. 7). As a result, the researcher gathered and analyzed information related to technology infusion and use the SAMR model as a tool to categorize findings. Furthermore, utilizing the SAMR model, the researcher examined how learning theories have evolved to incorporate connectivity and technology-based tools as variables within the teaching and learning process. Ultimately, this examination utilized research to analyze a variety of educational learning theories, and provide evidence to support a conceptual theory framework for technology-based formative assessment.

\section{Behaviorist Learning Theory}

Behaviorist learning theory, as referred to by researchers (Ally, 2004;

Boghossian, 2006; MacCallum \& Parsons, 2016; Ormrod, 2012), is the focus on external 
stimuli, where knowledge is validated by what can be observed independent of reflection, introspection, or internal processes. Behaviorists characterize conditioning as a verbal or physical response within activities where little subjectivity exists, and thus reinforces responses with grades or other measures (Alam, 2015; Boghossian, 2006). Burston (2015) stated, "The vast majority of educational apps are judged to be very traditional, predicated on transmission model learning, and behaviorist drill and practice approaches" (p. 45). As such, a behaviorist would examine technology as a function of access, and how introducing devices would affect learning. Utilizing technology for drill exercises and as a substitution for traditional practices both emphasize the behaviorist learning theory. In that, Puentedura (2013) explored the impact of technology directly as an impact on the behaviors of learning. While the study of formative assessment has yielded a positive impact on learning, Wiliam (2011) maintained initiatives in technology have failed to increase achievement as such efforts merely modified the method of providing equivalent instruction. This researcher examined the effectiveness of technological devices from the behaviorist perspective of increasing access and substituting traditional learning activities through increasing the ratio of students to devices.

Research on the effect of technology on student learning from a behaviorist perspective, has been traditionally grounded in experimental study of increasing the ratio of students to computing devices and its effect on student achievement. Although researchers (Cheung \& Slavin, 2013; Pilli \& Aksu, 2013; Shapley, Sheehan, Maloney, \& Caranikas-Walker, 2011) discovered that infusing technology into classrooms and instruction might have an impact on student learning, behaviorism, according to Boghossian (2006), is a function of changing behavior to obtain an increase in 
knowledge. MacCallum and Parsons (2016) provided examples within behaviorism to include, "quizzes, in class polling, discussion and question and answers" along with other skill-based learning activities (p. 176). Neebe and Roberts (2015) stated activities, such as those above, can be enhanced by using technology, specifically incorporating technological devices as a means to increase the frequency and timeliness of formative assessment feedback. Early theories on behaviorism and technology integration, according to researchers (Ally, 2004; Saettler, 2004), focused on the reinforcement of learning, removing teachers from traditional instructional situations, and asking learners to perform measured, observable, and quantifiable tasks. Within behaviorism, researchers (Saettler, 2004; Yilmaz, 2011) categorized technology-based formative assessment as a teacher-centered approach to learning, focusing on measurable behaviors utilizing technology as a tool for collecting acceptable responses, and thus, learning occurs independent of student reflection or thought. Rushton (2005) categorized formative assessment as independently affecting the behaviors of the teachers and students in such a way that learning occurs as a sequence of activities where summative assessment is derived from continuous formative assessments. Therefore, behaviorism-learning theory, by definition, would treat formative assessment, computing devices, and technology integration as separate variables with results independent of each other when incorporating within teaching and learning.

Researchers (Grant et. al., 2015; Lei \& Zhao, 2007) determined there is a lack of evidence to support a direct correlation between increasing access to computing devices and student learning. Alternatively, the inclusion and increase of computing devices shows gain in areas such as digital citizenship and computer fluency. Although low to 
moderate gains in achievement occurred when incorporating technology within instruction, external variables such as length of study, student and teacher attitude toward technology, skill set, and professional development are factors requiring further consideration when evaluating the effectiveness of technology integration on student achievement (Eyyam \& Yaratan, 2014; Inan \& Lowther, 2010; Kposowa \& Valdez, 2013). While evidence exists to support how behaviorist learning, theory applies to technology-based learning activities, research on how technology-based formative assessment impacts learning requires further exploration. According to researchers (Afshari, et. al., 2009; Offer \& Bos, 2009; Yelland, 2001), a disconnection exists between the design of applications, the pedagogy of classroom instruction and assessment, and the manner in which curriculum and instructional practice align with technology. Assertions of this nature provide evidence that substituting technology within instruction to change behaviors of teachers and learners is less effective (Jude et. al., 2014; Puentedura, 2013). If incorporating devices to the point of saturation, or toward a ratio of one device per student, shows a direct impact on student learning, then one could argue that technology devices change the behaviors of learning irrespective of other factors. "The success of the implementation of ICT (information and communication technology) is not dependent on the availability or absence of one individual factor, but is determined through the dynamic process involving a set up interrelated factors" (Afshari, et. al., 2009, p. 77). As such, it is the assertion of this researcher that behaviorism-learning theory is applicable in some contexts, yet does not completely encompass the ramifications of technology incorporation, specifically about technology-based formative assessment. 


\section{Cognitivist Learning Theory}

The transition from behaviorism to cognitivism came with the concession by researchers that not all learning is observable, and as such, behaviorism failed to explain the complexity of human abilities such as how individuals make sense of, and process, information (Ally, 2004; Berliner, 2006; Bransford et. al., 2000; Yilmaz, 2011). Cognitivist learning theory, according to Ally (2004), Winn and Snyder (1996), and Yilmaz (2011), emphasizes the qualitative change of thinking, reasoning, and meaning over time where students construct understanding through the resolution of comparing discrepancies between new information to prior knowledge. When considering activities, teachers should focus on creating meaningful context and instruction, such as including examples, illustrations, demonstrations, and feedback to provide mental models for students to reflect upon during the teaching and learning process (Fenstermacher \& Richardson, 2005; Yilmaz, 2011). Researchers within cognitive psychology asserted a correlation between student retention of concepts to their ability to formulate relationships between content and experiences, thus using meta-cognitive strategies that create opportunities for critical reflection (Ally, 2004; Stoyanova \& Kommers, 2002). Stoyanova and Kommers (2002) and Yilmaz (2011) further encouraged structured activities designed to make learning more memorable, such as providing the opportunity for learners to create their mapping as a guide to facilitate a deeper processing of information, while creating a collaborative learning environment. Incorporating technology through activities associated with cognitive learning theory provides an opportunity for learners to reflect, receive feedback on learning, and process results, thus expanding the scope of how technology integration impacts student learning beyond a 
substitution-level argument (Chang, Wu, Weng, \& Sung, 2012; Johnson, 2014).

Activities such as reciprocal teaching, anchored instruction, inquiry learning, discovery learning, and problem-based learning are the most distinctive methods when considering a cognitive perspective (Ertmer \& Newby, 2013; Yilmaz, 2011). These methods, such as exploration, summarization, case analysis, and investigation, are grounded in one or more elements of cognitive learning theory. As such, the cognitive learning approach to technology integration would be characterized as augmentation or modification of learning, utilizing technology to integrate the experiences, interactions, and mental processes of students (Cornelius \& Marston, 2009; Pfeiffer, Gemballa, Jarodzka, Scheiter, \& Gerjets, 2009).

Using the SAMR model, the utilization of technology to create and integrate opportunities for formative assessment serves as a substitute for other, more traditional, practices. However, Neebe and Roberts (2015) asserted that technology can enhance specific feedback strategies such as instant feedback, peer feedback, and student reflection feedback, each of which can be classified as highly effective (Cho, 2005; Magaña \& Marzano, 2014; Marzano, 2006). Within cognitive learning theory, technology used to enrich teaching and learning broadens the scope and application of the devices beyond substitution to include purposeful adjustment of learning activities (Ally, 2004; Romrell et. al., 2014). While technology does affect specific learning methods that are the focus of cognitivist learning theory, the primary implications for learning from a cognitivist approach exist in the augmentation dimension of the SAMR model. The features of technology-based formative assessment may augment learning, as they provide increased functionality to the process, such as increased speed of data collection, 
tabulation, and reconciliation toward an adjusted learning experience. Researchers (Ally, 2004; Ertmer \& Newby, 2013; Johnson, 2014) detailed facets of cognitive learning to focus on the transference and commitment of knowledge to memory, or information processing. Therefore, Ally (2004) outlined factors, which contribute to increased learning such as: 1) the location information is placed on the screen, 2) the use of advanced organizers, 3) designing specific pre-instructional questions, and 4) activation of pre-requisite knowledge.

Furthermore, differentiation based on learning styles and cognitive ability, providing additional supports, learner motivation, and utilizing techniques that allow learners to perceive and understand information are vital aspects to a technology-based lesson (Ally, 2004; Ertmer \& Newby, 2013; Yilmaz, 2011). While feedback is noted as a crucial element to processing and storing information, cognitive learning theory does not focus on the context in which technology-based formative assessment affects student learning beyond the opportunity for reflection (Alley, 2004; Yilmaz, 2011). Despite evidence suggesting the cognitive learning theory embraces elements that support formative assessment as being impactful on learning, this theory fails to connect technology as a vital variable in the teaching and learning process, leading this researcher to explore additional frameworks.

\section{Connectivism Learning Theory}

Traditional learning theories address elements of instruction and learning (i.e. cooperative learning, formative assessment, teacher collaboration) that may apply to technology-based formative assessment as a component of student learning (Magaña \& Marzano, 2014; Marzano, 2006, Wiliam, 2011). As previously noted, this researcher 
contends that it is necessary to make viable connections between traditional formative assessment and technology integration to examine the impact of technology-based formative assessment on learning. With the evolution of technology, theorists (Downes, 2008; Martin, 2015, Siemens, 2014) explored learning theories, which specifically focus on the integration and effect of technology on learning. In this section, this researcher analyzed connectivism as one such learning theory, generated because of what researchers (Johnson, 2014; Railean, 2017; Seimens, 2014) referred to as the digital revolution: the change in digital technologies, which influence instruction, processing, learning, and assessment. Furthermore, this examination analyzed the impact of educational technology on connectivism learning theory to determine if sufficient evidence exists to analyze the effect of technology-based formative assessment.

Connectivism learning theory, according to researchers (Conradie, 2014; Dunaway, 2011; Siemens, 2014), is a function of diverse learning networks utilizing external resources to develop the process of learning outside of behavioral or contextual experiences, which promotes enhancing the skill of learning through avenues provided by technology. Alternatively, behaviorism and cognitivism focus on the process of changing learner behavior or the context of processing information (Bell, 2011; Downes, 2008; Dunaway, 2011; Johnson, 2014), connectivism focuses on changing the definition and connection to knowledge utilizing technology as a medium (Bell, 2011; Downes, 2008; Siemens, 2014). According to Siemens (2014), connectivism is a learning theory that succeeds traditional learning theories, citing those theories as failing to broaden the scope of learning to include the impact of technology on knowledge acquisition, describe how learning occurs within organizations, or address how learning occurs outside of people. 
Researchers (Anderson \& Dron, 2011; Kop \& Hill, 2008) asserted that, within

connectivism, the role of the teacher is to help students create connections to knowledge sources distributed and stored in a variety of ways across information networks. Connectivism, as a process of learning, requires new thinking where the focus is not merely on what is learned, but should also promote access to information, synthesis of opinions and concepts, critical evaluation and methods increasing the capacity for learning (Quinton \& Allen, 2014; Smidt, Thornton, \& Abhari, 2017). Siemens (2014) asserted that as the amount of knowledge in the world increases, knowledge has a halflife - the time span between when knowledge is acquired to when it becomes outdated, or obsolete. Therefore, connectivism is a learning theory that seeks to define learning as a process of navigating and updating knowledge in real-time through the medium of technology. In this theory, knowing information becomes less important than the connection to information sets or networks that are continuously changing as new information becomes available (Dunaway, 2011; Siemens, 2014; Smidt, Thornton, \& Abhari, 2017).

Evaluating the connectivism learning theory through the SAMR model shows the effect of technology as being grounded in the redefinition stage, as the premise of connectivism is reconstructing the working definition of knowledge (Siemens, 2014). As such, technology is not a merely a substitution for traditional instructional methods but a substitute for how knowledge is acquired. Technology allows for a vast number of modified or augmented learning experiences to take place, which increases the ability of learners to access information (Anderson \& Dron, 2011; Dunaway, 2011; Siemens, 2014). Technology serves as medium to access information rather than a method for 
dissertation in practice, B. Jacobs

transference of knowledge. Although many researchers (Kropf, 2013; Siemens, 2014)

continue to support connectivism as a learning theory, this assertion has been challenged by other researchers (Barry, 2013; Bell, 2011), making an argument for connectivism as an instructional theory rather than a learning theory.

According to Kropf (2013), an instructional theory is grounded in learning theory based on empirical findings to formulate material design and resources necessary to increase learning. According to Driscoll (2005), learning theory explained how learning occurs through constructs that link the performance of learning to the perceived cause for increased performance. Assertions of this nature led Kropf (2013) to conclude that connectivism can serve as both a learning theory and instructional theory, however, could not support the true succession of traditional learning theories, as connectivism was often found to work in conjunction with behaviorist, cognitivist, and constructivist learning theories. Establishing connectivism as a learning theory supports the claim that technology applications play an incremental and unique role in the $21^{\text {st }}$-century learning environment. However, Ozan and Kesim (2011) asserted when considering technologybased formative assessment, connectivism primarily focuses on social components of learning, and how learner connectivity creates nodes linking learners to external sources of knowledge. Researchers (Black \& Wiliam, 2009; Nicol \& Macfarlane-Dick, 2006; Sadler, 1998; Wiliam; 2011) defined formative assessment as any activity designed and intended to provide feedback to a learner about their performance in correspondence to their learning and used to modify teaching and learning activities. Where connectivism serves as a framework for how, and when, to incorporate technology, Siemens (2014) asserted the principals of connectivism focus on an ever-changing climate of information 
by which the learner is focused on internalizing a process rather than committing knowledge to memory. Researchers (Black \& Wiliam, 2009; Cowie \& Bell, 1999; Wiliam, 2011) indicated formative assessment feedback has a direct impact on transference and retention of desired objectives. As such, the connectivist learning theory succeeded in closing the gap between traditional learning theories and technology, although failed to consider the impact of formative assessment feedback.

\section{Mobile Learning Theory as a Conceptual Framework}

In previous sections, this researcher found evidence to support impact of technology on learning through the behaviorist, cognitivist, and connectivist learning theories. Although connections to technology are relevant, the examination of each learning theory failed to make a specific connection regarding the impact technology has on learning as a function of enhancing formative assessment feedback. Based on these findings, this researcher contends the analysis of Mobile Learning Theory (MLT) yields a proper framework for analyzing how technology incorporates components of the learning above theories to elevate learning experiences beyond what is available, absent of technological tools and applications. Additionally, this researcher examined the impact of technology on learning through enhancing formative assessment as a function of methodological and pedagogical frameworks within MLT.

To evaluate MLT, it is important to clarify the difference between electronic and mobile learning. Electronic learning (e-Learning) has evolved in definition as technologies have evolved, thus creating difficulty in adopting a single definition (Sangra, Vlachopoulos \& Cabrera, 2012). Corbeil and Valdes-Corbeil (2007) identified e-Learning as the facilitation and support of learning through information and 
dissertation in practice, B. Jacobs

communication technologies. Generally, e-Learning is referred to regarding

environmental factors, or tools, contributing to learning or used to "create, order, store, disseminate, revise, and maintain all manner of materials" (Kirschner, 2004, p. 40). Nichols (2003) defined e-Learning within the context of information analysis through web-based or web-capable technological tools, believing some level of interactivity should be involved.

Further, at a more constructivist level, Tavangarian, Leypold, Nölting, Röser, and Voigt (2004) asserted that e-Learning should include a transformational component to learning experiences which expand beyond the procedural component, thus creating the conditions for further knowledge construction. E-Learning, used as a term to describe the conditions of learning using technology based tools, has advanced the focus of researchers to determine the different contexts in which technology can be used to achieve or enhance learning. As such, Sangra, Vlachopoulos and Cabrera (2012) concluded that e-Learning is comprehensively more conceptual, and the definition may vary when considering its use as a specific technology, method of accessing knowledge, as a communication tool, or concerning a specific paradigm within the educational component it is intended to enhance.

Georgiev, Georgieva, and Smrikarov (2004) expanded on the concept of eLearning and stated as mobile technology devices have evolved, mobile learning, as a more specific and measurable form of e-Learning was established. Traxler (2007) stated mobile learning differs from e-Learning in that it provides context-specific learning to learners without the limits of a specific time or location, and thus outlined several ways mobile learning expands on the concept of tethered e-Learning. However, Traxler further 
alluded to the potential that once all functions of e-Learning are realized within mobile devices, mobile learning would then be realized as the more encompassing theory. Mobile Learning Theory has evolved over time, as the benefits of embedding technology within the teaching and learning process have been explored (Wu et. al., 2012). Early definitions of MLT focused on the use of particular devices, which according to researchers become outdated over time, and fail to address the transformative potential within the practice of teaching and learning (Crompton, 2013; Paschler, Bachmair, \& Cook, 2011). As studies on the subject expanded, researchers (Sharples, Taylor, \& Vavoula, 2007) narrowed the scope of MLT to focus on the collaborative and communicative effects of interactive technologies on how knowledge is attained. Farrow (2011) addressed mobile learning from multiple contexts and identified the necessity of considering ethical and moral factors within technology integration.

Further exploration of MLT expanded on the theory broadly in nature, referring to the impact of technology on learning in mobile and ubiquitous situations (Cope \& Kalantzis, 2009; Ogata \& Yano, 2004; Hwang \& Tsai, 2011). Because of a meta-analysis of literature on MLT, Hwang and Wu (2014) asserted there exists positive and promising effects of mobile learning devices on "student learning achievements, motivation, and interests" (p. 83). As mobile devices have increased in accessibility and functionality, research within MLT has evolved concurrently to address the realities and implications of teaching and learning with mobile devices and applications designed for specific contexts and uses.

According to Chee, Yahaya, Ibrahim and Hasan (2017), "research findings on using mobile devices in different learning environments have exemplified their ability to 
enhance students' learning knowledge" (p. 114). Peters (2007) stated the use of mobile learning devices enables ubiquitous learning by expanding learning opportunities beyond fixed locations, and therefore creates a variety of different methods of working and learning. Mobile learning enhances student learning by providing individualized access to information, immediacy in reflection, expedient and collaborative interaction, and convenience (Kukulska-Hulme, Sharples, Milrad, Arnedillo-Sanchez, \& Vavoula, 2009; Kynaslahti, 2003). These opportunities utilizing both contained and online resources, specifically incorporating learning within mobile devices, provides a venue for learning to be situational, self-monitored, or self-directed depending on the conditions (Nicol \& Macfarlane-Dick, 2006; Cheon, Lee, Crooks, \& Song; 2012; Wong, 2016).

As explored in the previous section, Mobile Learning Theory has been defined and researched in a variety contexts and settings. Recently, and for the purpose of this study, researchers (Crompton, 2013; Hwang \& Chang, 2011; Reeves et. al., 2017; Romrell et. al., 2014; Xie, 2016) outlined components of MLT as personalized, situated, and portable learning that is continuous in nature, thus extending learning experiences beyond the classroom using personal electronic devices. Furthermore, the construct of MLT outlines the potential impact of mobile devices on learning as a function of transforming learning into connected, convenient, real world, and authentic experiences with methods not previously envisioned (Cochran, 2016; Romrell et. al., 2014; Sharples et. al., 2010; Xie, 2016). According to Dyson, Litchfield, Raban, and Tyler (2009), the interactive systems available within mobile learning de-emphasize traditional teachercentered models and encourage active learning processes. In turn, this allows for seeing an increasing attention span, motivation, and the ability of teachers to adapt learning 
experiences to the needs of students. Additionally, MLT provides a platform for students to obtain and learn information outside of the constructs of traditional pedagogy (Dyson et. al., 2009; Xie, 2016). Researchers have identified strategies within MLT enhance formative assessment and feedback to promote learning; some examples include Flipped Classroom for Active Learning (Wong, 2016), Cross-Contextual Learning (Xie, 2016), and the use of mobile devices to provide authentic and virtual learning experiences (Cochran, 2016).

MLT, evaluated using the SAMR model, allows instructors to classify MLT experiences comparatively with traditional learning experiences (Jude et. al, 2014). Researchers of the SAMR evaluation model (Jude et. al., 2014; Puentedura, 2013; Romrell et. al., 2014) asserted the purpose for implementing MLT technologies could vary depending on the desired outcome. As a result, methodological frameworks have been utilized to analyze the impact of technology on teaching and learning (Burston, 2015; Jude et. al., 2014; Romrell et. al., 2014). When referencing technology-based formative assessment, utilizing technology augments the teaching and learning process by increasing the functionality of formative assessment feedback, therefore enhancing student learning through reflection, collaboration, real-time adjustment to learning activities (Hwang \& Chang, 2011; Kihoza, Zlotnikova, Bada, \& Kalegele, 2016; Pachler, Daly, Mor, \& Mellar, 2010). Wong (2016) further asserted technology generates feedback as a function of formative assessment, provides a platform for students to regulate their performance, and motivates student learning within self-regulated learning experiences. Researchers (Burns et. al., 2010; Dyson \& Frawley, 2016; Lee et. al., 2012) examined technology-based formative assessment to analyze the impact of technology on 
formative assessment and learning, concluding that TEFA programs positively influence student achievement in a variety of settings.

A further examination on the impact of formative assessment yielded the importance of feedback within the teaching and learning process. Researchers (Heitink, Van der Kleij, Veldkamp, Schildkamp, \& Kippers, 2016) asserted formative assessment should include "substantial, constructive and focused feedback" (p. 50). Feedback is an essential element of formative assessment, where according to Duijn et al. (2017), feedback should come from a credible source, address strengths and improvement areas, provide specific instructions for improvement, be provided immediately and should occur regularly including a follow-up conference. According to Xie (2016), utilizing mobile learning strategies to provide feedback and tips allows students to obtain feedback from multiple locations, contains individualized learning content specific to student response, and occurs in a nearly instantaneously fashion. While formative assessment feedback is widely considered beneficial (Black \& Wiliam, 2009; Dunn \& Mulvenon, 2009; Wiliam, 2011), feedback can be detrimental to the engagement in learning activities when it is significantly delayed (Bhagat \& Spector, 2017). Furthermore, Bhagat and Spector (2017) asserted, "Timely and informative feedback is essential for formative assessment to be effective" (p. 312). As such, a lack of meaningful feedback promptly may create misconceptions, or barriers to learning which can be difficult for learners to overcome in later stages of the learning process (Bhagat \& Spector, 2017). Also, Faber, Luyten, and Visscher (2017) concluded feedback has a positive effect within mathematics achievement and motivation utilizing Snappet, a digital formative assessment tool. 
dissertation in practice, B. Jacobs

As previously stated, the SAMR model provides a framework for classifying activities when utilizing technology, allowing instructors to identify a purpose for mobile technology integration (Jude et. al., 2014; Romrell et. al., 2014). Researchers of the SAMR model (Jude et. al., 2014; Puentedura, 2013; Romrell et. al., 2014) asserted the use of technology can enhance and transform learning experiences and directly impact student learning and achievement when implemented beyond the substitution level. Analyzing the results of mobile learning activities to increase the quality and regularity of formative feedback to students will maximize the effect of MLT activities on student learning (Cochrane, 2012; Wong, 2016). Furthermore, Dyson and Frawley (2016) stated the implementation of MLT dramatically improves formative assessment feedback and marks. In addition, researchers (Bennet \& Gitomer, 2009; Bhagat \& Spector, 2017) asserted the use of technology will make formative assessment more informative, efficient, and cost-effective, and therefore increases the impact of formative assessment on student learning. Ultimately, utilizing the SAMR model to evaluate MLT provides a framework to examine methodologies and how augmentation, modification, and redefinition of activities using technology enhances formative assessment.

Examination of formative assessment in conjunction with the SAMR model, established a variety of applicable thematic pedagogies. According to researchers (Burns et. al., 2010; Lee et. al., 2012), TEFA, which for the purpose of this analysis includes the Technology Enhanced Formative Evaluation (TEFE), results in improvement of student learning while at the same time decreasing the burden placed on teachers. Within TEFA, Classroom Response Systems (CRS) technology provides concrete, implementable, principle-based and effective teaching practices that enhance formative assessment 
(Beatty \& Gerace, 2009). Rochelle, Penuel, and Abrahamson (2004) identified four constructs to unify CRS-Based instruction to the broad context of education research and what can occur when CRS instruction is implemented. Those constructs are: a) formative assessment, b) driving discussion by important conceptual contrasts, c) shifting to mastery-oriented motivational incentives, and d) harnessing diversity for generativity. Feldman and Capobianco (2008) expanded on the four constructs by further identifying the influence of curriculum integration and incorporating methods for constructing formative assessment items as components relevant to impacting teachers' practice. Furthermore, to fully integrate CRS within the teaching and learning process, teachers must understand the nature of formative assessment and actively collaborate with colleagues to reflect on the effectiveness of plans (Feldman \& Capobianco, 2008). Although researchers (Beatty \& Gerace, 2009; Feldman \& Capobianco, 2008; Lee, et. al., 2012) have focused on studies specifically tied to CRS, utilizing TEFA pedagogy to enhance formative assessment results expanded beyond substituting devices for traditional activities. To bridge the gap between CRS and other mobile technologies, therefore expanding the scope of how technology encourages augmentation, modification and redefinition of learning experiences, studies have yielded similar results when examining mobile learning devices and applications beyond CRSs (Hwang \& Chang, 2011). Martin (2015) asserted technologies could be used as a pedagogical tool, specifically overlapping the areas of pedagogical knowledge and content knowledge, and allowing for infusion of technology as a resource within traditional teaching methodologies. Martin expands the concept that technological knowledge, pedagogical knowledge, and content knowledge viewed as individual processes and objectives, or 
could overlap within many contexts. It is when the contexts of each of these knowledge types overlap in nature that MLT can be most evident and beneficial to learning.

As stated in previous sections, it is clear research on mobile technologies and formative assessment is continuing to yield results in support of utilizing mobile devices to enhance learning (Van der Kleij, Feskens, \& Eggen, 2015). When connecting MLT and formative assessment, Hwang \& Chang (2011) explored formative assessment-based mobile learning, an approach utilizing mobile devices to provide immediate feedback to students during learning activities. Further, Conejo, Garcia-Vinas, Gaston and Barros (2016) employed a web-based tool to incorporate TEFA that provided elaborated immediate feedback, and thus concluded students utilizing the designed tool outperformed those utilizing traditional formative assessment methods. While research on the effects of mobile learning on student achievement are found to be beneficial, Afshari et al. (2009) asserted teacher ability within technology integration, quality of hardware and software resources, available time for collaboration within staff members, and lack of training and support for teachers within technology infusion are barriers to teacher implementation of mobile learning strategies. Furthermore, according to Ekanayake and Wishart (2014), the variables associated with teacher training and support have not been explored with fidelity within mobile-learning research.

\section{Summary}

Theories on the acquisition of knowledge yielded a variety of methodologies related to how information is accessed, retained, and applied. Learning theories evaluated through the behaviorist, cognitivist, and constructivist lenses provided insight on how learners process and retain information (Luis \& D'Cunha, 2014; MacCallum \& 
Parsons, 2016; Siemens, 2014). An examination of the effects of technology on student learning have yielded substantial results in educational research, including the proposition of new learning theories such as distance learning, e-Learning (Corbeil and Valdes-Corbeil, 2007; Kirschner, 2004; Sangra, Vlachopoulos \& Cabrera, 2012) and connectivism (Siemens, 2014). MLT and additional pedagogies specific to formative assessment such as TEFA and FAML (Burns et. al., 2010; Lee et. al., 2012; Hwang \& Chang, 2011) expanded formative assessment research concerning ubiquitous and situated learning experiences. Technology and technology-based tools within education were not intended to replace traditional learning theories or research on the effectiveness of traditional practice. They are a direct result of the introduction and advancement of technological devices and applications developed to enhance what research has already revealed about human learning in the context of a technology-rich environment.

Although connectivism is one theory which provided a construct for analyzing learning as a function of connectivity to information (Conradie, 2014; Dunaway, 2011; Siemens, 2014), it did not provide a proper connection to the human element of feedback. Primarily, connectivism learning theory emphasized the transformation and redefinition of knowledge, with knowledge being accessible in real-time and expanding as online literature evolves (Siemens, 2014). Research regarding the impact of formative assessment on learning is extensive, prescribing to practitioners the necessity of embedding timely and meaningful feedback within each lesson with specific intentionality (Black \& Wiliam 1998; Cowie \& Bell, 1999; Hwang \& Chang, 2011; Nicol \& Macfarlane-Dick, 2006; Reeves, Gunter \& Lacey, 2017; Wiliam, 2011). Therefore, examining the impact of technology-based formative assessment feedback on student 
dissertation in practice, B. Jacobs

learning necessitated an investigation beyond traditional learning theories to include learning theories, which accounted for technology integration.

Mobile learning, according to researchers (Crompton, 2013; Hwang \& Chang, 2011; Xie, 2016) has evolved as research within the field has expanded beyond the concept of e-Learning. Further, research expanded to include situational, self-regulated, and independent of location (Hwang \& Chang, 2011; Reeves et. al., 2017; Romrell et. al., 2014; Xie, 2016). As specific devices became outdated or obsolete, research then broadened to consider the universal effects of ubiquitous mobile learning, creating a need to research how mobility of ICT's impacted the acquired knowledge and creation of learning experiences. As results varied within different contexts, research has been more focused on the transformation of teaching and learning through technology within specific content areas and age groups, thus increasing the validity and transferability of findings. As such, MLT provided a connection to augmenting, modifying, and redefining formative assessment learning experiences to elevate already proven strategies and enhance student learning. 


\section{INTRODUCTION}

Researchers have attempted to make connections between practices within teaching and learning, and how the use of technology can enhance or deplete their effectiveness (Andersson \& Palm, 2017; Chappuis, 2015; Pahomov, 2014; Wiliam, 2011). Before Johnson (2014), Railean (2017), and Siemens (2014) described the 'digital revolution', educational standards, generically if not specifically, incorporated the preparation of students for success in the workforce. However, not previously addressed within these education standards was preparing $21^{\text {st }}$ Century workers within technologybased initiatives, as projections by economists have indicated the growing need for workers to have specific skills in technology (Alismail \& McGuire, 2015; Herman, 1999). When investigating technology within education, elements such as funding, program initiatives, and the introduction of specific technology standards indicate a unified belief within the educational community that technology is a necessary component of the educational process (Czaja, Charness, Fisk, Hertzog, Nair, Rodgers, \& Sharit, 2006; Martin, 2015). The National Education Technology Plan (2016) stated technology could enhance proven instructional methods and increase the potential of learning while consolidating the resources necessary to accomplish the same objectives. The national movement in support of technology infusion resulted in itemized plans by school districts to increase technological hardware and software, provide professional development for staff on effective technology-based instruction, and develop protocols for evaluating the effect of technology-based initiatives (Niederhauser \& Lindstrom, 2018; Schmidt-Crawford, Lindstrom \& Thompson, 2018). 
dissertation in practice, B. Jacobs

Through an exhaustive review of the literature, a reasonable gap existed between the impact technology has on formative assessment feedback, and thus on student learning. As such, it was this researcher's focus to determine whether technological tools enhance strategies to increase the capacity of student learning within $7^{\text {th }}$ and $8^{\text {th }}$ grade mathematics. Therefore, the overarching focus of this inquiry was to analyze the impact technology has on learning as it pertains to increasing classroom instruction and efficiency, timeliness of feedback, collaboration and communication, and overall student achievement on specific learning objectives. The following sections will describe the setting, present the results, and the implications, along with recommendations through an executive summary.

\section{Research Questions}

The following research questions guided the study:

1. How do technology-based tools and applications impact the way teachers gather, analyze, and utilize information obtained from formative assessments to impact student learning?

2. How does the professional development and training of teachers and administrators specific to technology-based tools and applications (IXL, ALEKS, Schoology, or other applications) impact the way teachers adapt instructional learning opportunities?

3. How do technology-based formative assessment resources impact the frequency and quality of feedback utilized by teachers to enhance teaching and learning? 


\section{Connection to Conceptual/Theoretical Framework}

This researcher determined Mobile learning theory (MLT) as the conceptual framework that allowed for examining the augmentation or redefinition of learning activities, which researchers (Puentedura, 2013; Romrell et. al., 2014) described as expanding learning experiences beyond what is capable without technology. Through the utilization of technology, MLT allows learning to transform, creating instant and expansive opportunities within and outside of the classroom for learning to occur (Romrell et. al., 2014, Sharples, Taylor, \& Vavoula, 2007). Researchers also asserted through use of technology-based tools that learners could obtain information in different contexts, thus allowing for a more effective transfer and commitment of information to memory (Hwang \& Chang, 2011; Pachler et. al. 2010; Puentedura, 2013; Romrell et. al., 2014). Proponents of MLT further emphasized incorporating feedback and formative assessment is an effective and impactful variable within the teaching and learning teaching cycle (Burns, Klingbeil, \& Ysseldyke, 2010; Dyson \& Frawley, 2016; Lee, Feldman, \& Beatty, 2012), and as such was the conceptual framework utilized in this investigation.

\section{Setting and Participants}

This researcher selected a second tier suburban school district (District) located outside of Kansas City, Missouri, as the case for this study. Purposefully selected for this study was the school district, because it is a highly innovative district with regard to technology initiatives. The District is composed of three middle schools, containing approximately twenty-eight certified teachers employed to implement curricular programs and provide mathematics instruction and assessment to approximately 2850 
dissertation in practice, B. Jacobs

middle school students (Department of Elementary and Secondary Education, 2018).

Included in Table 2 is the description of the organization.

Table 2

Description of Organization Structure of the Participants

\begin{tabular}{|c|c|c|c|c|c|c|c|}
\hline \multirow[b]{2}{*}{$\begin{array}{c}\text { Schoo } \\
1\end{array}$} & \multicolumn{2}{|c|}{ Student } & \multicolumn{3}{|c|}{ Teacher } & \multicolumn{2}{|c|}{ Principal } \\
\hline & Enrollment & $\begin{array}{c}\text { F/R } \\
\text { Lunch }\end{array}$ & $\begin{array}{c}\text { Total } \\
\text { Teacher } \\
\text { s }\end{array}$ & $\begin{array}{c}\mathrm{Av} \\
\mathrm{g} \\
\operatorname{Exp}\end{array}$ & $\begin{array}{l}\text { Advanced } \\
\text { Degrees }\end{array}$ & $\begin{array}{l}\text { Avg } \\
\text { Yrs at } \\
\text { Site }\end{array}$ & $\begin{array}{l}\text { Highest } \\
\text { Degree }\end{array}$ \\
\hline A & 841 & $27 \%$ & 68 & 14 & $86.8 \%$ & 14 & Doctorate \\
\hline B & 934 & $25 \%$ & 63 & 12 & $87.3 \%$ & 3 & $\begin{array}{c}\text { Education } \\
\text { al }\end{array}$ \\
\hline $\mathrm{C}$ & 1134 & $11 \%$ & 69 & 14 & $82.6 \%$ & 7 & Doctorate \\
\hline
\end{tabular}

Note: $\mathrm{N}=5$ interview participants; 3 building level principals; $\mathrm{N}=4$ focus groups; participants 1-15 were teachers from 3 school sites, participants 16-21 were district level instructional level support staff

Within this single case study, the researcher utilized three sites specific to $7^{\text {th }}$ and $8^{\text {th }}$ grade mathematics instruction, the three middle schools. Each middle school site has a three-person administrative unit, including a Principal and two Assistant Principals.

Within these three schools, conducted was an interview with an administrator from each site, as well as appropriate district level administrators to gather information related to the research questions within this study. Provided in Table 3 is a description of the administrative participants. 
Table 3

Administrator Interview Participant Demographics

\begin{tabular}{ccccccc}
\hline Participant & Jender & $\begin{array}{c}\text { Age } \\
\text { Range }\end{array}$ & $\begin{array}{c}\text { Years of } \\
\text { Experience }\end{array}$ & $\begin{array}{c}\text { Years in } \\
\text { Leadership }\end{array}$ & Highest Degree & $\begin{array}{c}\text { Certified } \\
\text { (Yes or } \\
\text { No) }\end{array}$ \\
\hline 1 & F & $40-50$ & 26 & 18 & Doctorate & Yes \\
2 & F & $30-40$ & 12 & 4 & $\begin{array}{c}\text { Educational } \\
\text { Specialist }\end{array}$ & Yes \\
3 & M & $40-50$ & 23 & 14 & $\begin{array}{c}\text { Educational } \\
\text { Specialist }\end{array}$ & Yes \\
4 & F & $50-60$ & 24 & 15 & Doctorate & Yes \\
5 & M & $40-50$ & 22 & 6 & Doctorate & Yes \\
\hline
\end{tabular}

Note: Years of experience and leadership are not specifically years in the district

Subsequently, conducted were focus groups at each site with a representative group of $7^{\text {th }}$ and $8^{\text {th }}$ grade math teachers. Illustrated in Table 4 is a description of the teacher participants from each site. 
dissertation in practice, B. Jacobs

Table 4

Teacher Focus Group Participant Demographics

\begin{tabular}{|c|c|c|c|c|c|c|}
\hline Participant & Site & Gender & $\begin{array}{c}\text { Age } \\
\text { Range }\end{array}$ & $\begin{array}{c}\text { Years of } \\
\text { Experience }\end{array}$ & Highest Degree & $\begin{array}{c}\text { Certified } \\
\text { (Yes or } \\
\text { No) }\end{array}$ \\
\hline 1 & $\mathrm{~A}$ & $\mathrm{~F}$ & $30-40$ & 14 & Masters & Yes \\
\hline 2 & $\mathrm{~A}$ & $\mathrm{~F}$ & $30-40$ & 15 & Masters & Yes \\
\hline 3 & A & $\mathrm{F}$ & $30-40$ & 24 & Masters & Yes \\
\hline 4 & A & $\mathrm{F}$ & $40-50$ & 13 & Masters & Yes \\
\hline 5 & $\mathrm{~A}$ & M & $40-50$ & 14 & Masters & Yes \\
\hline 6 & B & M & $40-50$ & 7 & Masters & Yes \\
\hline 7 & $\mathrm{~B}$ & $\mathrm{~F}$ & $40-50$ & 7 & Masters & Yes \\
\hline 8 & B & $\mathrm{F}$ & $40-50$ & 3 & Bachelors & Yes \\
\hline 9 & $\mathrm{~B}$ & $\mathrm{~F}$ & $30-40$ & 13 & Masters & Yes \\
\hline 10 & B & $\mathrm{F}$ & $40-50$ & 19 & Educational Specialist & Yes \\
\hline 11 & $\mathrm{C}$ & $\mathrm{F}$ & $30-40$ & 20 & Masters & Yes \\
\hline 12 & $\mathrm{C}$ & $\mathrm{F}$ & $40-0$ & 25 & Masters & Yes \\
\hline 13 & $\mathrm{C}$ & $\mathrm{F}$ & $30-40$ & 17 & Masters & Yes \\
\hline 14 & $\mathrm{C}$ & $\mathrm{F}$ & $40-50$ & 29 & Masters & Yes \\
\hline 15 & $\mathrm{C}$ & $\mathrm{F}$ & $30-40$ & 14 & Masters & Yes \\
\hline
\end{tabular}

Note: Years of experience and leadership are not specifically years in the district

Finally, the researcher attempted to collect data specific to district level curriculum, instruction, and technology specialists through a focus group. Provided in Table 5 is a description of district level support participants. 
dissertation in practice, B. Jacobs

Table 5

District Specialist Focus Group Participant Demographics

\begin{tabular}{|c|c|c|c|c|c|c|c|}
\hline Participant & Jender & $\begin{array}{c}\text { Age } \\
\text { Range }\end{array}$ & $\begin{array}{c}\text { Years of } \\
\text { Experience }\end{array}$ & $\begin{array}{c}\text { Years in } \\
\text { Current } \\
\text { Role }\end{array}$ & Highest Degree & Current Role & $\begin{array}{l}\text { Certified } \\
\text { (Yes or } \\
\text { No) }\end{array}$ \\
\hline 1 & $\mathrm{~F}$ & $\begin{array}{l}40- \\
50\end{array}$ & 27 & 15 & Doctorate & Instruction & Yes \\
\hline 2 & $\mathrm{~F}$ & $\begin{array}{l}30- \\
40\end{array}$ & 15 & 1 & Masters & Curriculum & Yes \\
\hline 3 & M & $\begin{array}{l}30- \\
40\end{array}$ & 10 & 3 & Masters & Technology & Yes \\
\hline 4 & $\mathrm{~F}$ & $\begin{array}{c}50- \\
60\end{array}$ & 22 & 5 & Masters & Curriculum & Yes \\
\hline 5 & M & $\begin{array}{c}30- \\
40\end{array}$ & 15 & 3 & $\begin{array}{l}\text { Doctorate } \\
\text { Candidate }\end{array}$ & Technology & Yes \\
\hline 6 & $\mathrm{~F}$ & $\begin{array}{l}30- \\
40\end{array}$ & 18 & 5 & Masters & Instruction & Yes \\
\hline
\end{tabular}

Note: Years of experience and leadership are not specifically years in the district

Presented in the following section are the results of the study, including a qualitative analysis of the data. Transcribed, coded, and analyzed to determine emerging themes with respect to the three research questions were the data sets.

\section{Presentation of the Findings}

\section{Research Question One}

How do technology-based tools and applications impact the way teachers gather, analyze, and utilize information obtained from formative assessments to impact student learning?

Within the teaching and learning cycle, district-level administrators and specialists, in conjunction with building leaders, communicated it is an expectation that 
teachers gather, analyze and utilize information to adjust and adapt to learning experiences. On this topic, Principal B stated,

We expect our teachers to take that formative assessment and then develop their learning plan forward and that is part of making sure our formative assessments are not at the very end of a unit, that we have a pulse of where our kids are. Added, Leader A stated it is a focus of district support specialists to find resources for teachers which are "going to elicit data that we can look at that's real-time data in a quick, meaningful way to turn around and utilize to inform instruction." As a result, curriculum specialists, with the support of instructional and technology specialists each referenced the resources and training as an essential element of the process, siting training sessions and web sites outlined with technology-based tools, which they have provided to the teachers.

Located on the curriculum website, as noted by this researcher, were technologybased tools and applications with instructions on how to create and imbed formative assessment within a unit or standard utilizing specific technology-based applications. In addition, district leaders, curriculum specialists, and principals all referenced the availability for training and support by specialists. Principal A described professional development opportunities by the technology specialist,

(Specialist C) will actually sit for an entire day and in one of our conference rooms. While teachers can kind of rotate in and out and really focus on their planning time on what they specifically want to know for their classroom without having to always only get what they would get in a large group that's been kind of prescribed for them 
Specialist A, who serves in a similar role as Specialist C, stated, "I meet appointment by appointment basis. I do schedule times to be in building, usually on Tuesdays and Thursdays, to spend an entire time, whether it's an elementary, middle, or high school building, to be with them." Ultimately, the goal and expectation of the district leaders, specialists and principals, as suggested by the evidence collected, and noted examples in Table 6, is for teachers to utilize information from formative assessment to impact learning experiences, and for the district to provide resources, training and support to teachers that inform instruction. 
Table 6

Formative Assessment, Technology-based Formative Assessment, Professional DevelopmentPrincipal Interviews - Illustrative Quotes

\begin{tabular}{|c|c|c|c|}
\hline $\begin{array}{l}\text { Principal } \\
\text { Participant }\end{array}$ & Formative Assessment & $\begin{array}{l}\text { Technology-Based } \\
\text { Formative Assessment }\end{array}$ & $\begin{array}{l}\text { Professional } \\
\text { Development and } \\
\text { Support }\end{array}$ \\
\hline $\begin{array}{l}\text { Principal } \\
\text { A }\end{array}$ & $\begin{array}{l}\text { "Formative assessment can } \\
\text { fit into any and all of those } \\
\text { particular instructional } \\
\text { strategies. And so we } \\
\text { always tell teachers that } \\
\text { you really shouldn't go } \\
\text { more than a few minutes } \\
\text { without having some kind } \\
\text { of feedback opportunity." }\end{array}$ & $\begin{array}{l}\text { "I've seen teachers, as } \\
\text { they're gathering that } \\
\text { feedback, they'll just } \\
\text { realize, "Okay. This isn't } \\
\text { working. We need to } \\
\text { stop, back up, and redo } \\
\text { this because you guys are } \\
\text { not at the point that I } \\
\text { thought you would be." }\end{array}$ & $\begin{array}{l}\text { "[Specialist C ] will } \\
\text { actually sit for an entire } \\
\text { day and in one of our } \\
\text { conference rooms, and } \\
\text { teachers can kind of } \\
\text { rotate in and out and } \\
\text { really focus on their } \\
\text { planning time, on what } \\
\text { they specifically want } \\
\text { to know for their } \\
\text { classroom }\end{array}$ \\
\hline $\begin{array}{l}\text { Principal } \\
\text { B }\end{array}$ & $\begin{array}{l}\text { "We expect our teachers to } \\
\text { take that formative } \\
\text { assessment and then } \\
\text { develop their learning plan } \\
\text { forward and that's part of } \\
\text { making sure our formative } \\
\text { assessments are not at the } \\
\text { very end of a unit, that we } \\
\text { have a pulse of where our } \\
\text { kids are." }\end{array}$ & $\begin{array}{l}\text { "I think it enriches the } \\
\text { feedback they get from } \\
\text { their peers and also the } \\
\text { feedback they give to } \\
\text { their teachers as well" } \\
\text { "...you aren't confined to } \\
\text { the parameters of the } \\
\text { school day." }\end{array}$ & $\begin{array}{l}\text { "When it's not } \\
\text { mandatory, often times } \\
\text { teachers will find other } \\
\text { things to do than to take } \\
\text { that PD if it's not } \\
\text { something they're really } \\
\text { into. But I would say } \\
\text { the teachers that have } \\
\text { taken advantage of } \\
\text { those opportunities, } \\
\text { especially in formative } \\
\text { assessment" }\end{array}$ \\
\hline $\begin{array}{l}\text { Principal } \\
\text { C }\end{array}$ & $\begin{array}{l}\text { "It would be an expectation } \\
\text { that if I'm monitoring the } \\
\text { class and I'm gathering } \\
\text { feedback, that there's some } \\
\text { corrective action taken." } \\
\text { "The talking point with } \\
\text { teachers is what was your } \\
\text { adjustment to your } \\
\text { instruction or to the class } \\
\text { based on the feedback that } \\
\text { you were getting from } \\
\text { students?" }\end{array}$ & $\begin{array}{l}\text { "I think in some cases, it } \\
\text { may shorten the amount } \\
\text { of time that teachers } \\
\text { spend on a topic because } \\
\text { technology opens up } \\
\text { opportunities to flip } \\
\text { lessons to have students } \\
\text { engage in some learning } \\
\text { outside of the classroom } \\
\text { prior to coming to class." }\end{array}$ & $\begin{array}{l}\text { "Putting a device in a } \\
\text { teacher's hand without } \\
\text { training is a waste of } \\
\text { time. And so in most } \\
\text { places when they move } \\
\text { to technology- } \\
\text { based classrooms, } \\
\text { there's an investment in } \\
\text { teacher learning before } \\
\text { the devices are ever } \\
\text { rolled out to the } \\
\text { students" }\end{array}$ \\
\hline
\end{tabular}


dissertation in practice, B. Jacobs

\section{Gathering and Analyzing Data}

Throughout this process, the researcher discovered three types of evidence in reference to gathering and analyzing data from technology-based formative assessment. The first type is in how teachers utilize technology to gather and analyze data in an augmented, modified or redefined learning experience. Second, the increased availability of feedback provided by technology-based tools encouraged teachers to infuse learning experiences for students outside of the school setting. Contained in the following section is the collection of both data sets.

Evident throughout the collected data was the commitment of the district to amplify teaching and learning experience by utilizing technology infusion as more than a substitution for traditional learning practices. As evidenced in the districts five year plan to "Fully implement and utilize the district's learning management system (LMS) to design lessons, promote access to high quality and relevant digital content, assess learning in order to support personalization and ownership of student learning by everyone involved in a student's education, both inside and outside of the classroom." Leader A, who oversees instructional operations for all levels, explained, "So the goal is redefinition, and that only occurs through teachers' understanding of how the use of technology devices, or in this case relative to your study, how technology-based formative assessments can assist with and really help propel student learning." While redefinition is the ultimate goal, the district technology administrator, Leader B, expanded by stating, "We're using technology to blend instruction to where sometimes we're substituting, sometimes modifying, sometimes we're redefining, but we're going to hit it somewhere in there." Leader A continued, 
dissertation in practice, B. Jacobs

There is a place for all four of those (substitution, augmentation, modification, redefinition) across the curriculum, across a curricular year and a content area, to be utilized with the teacher for student benefit. So you're not necessarily looking for all of those to be in place all the time.

Although the goal is redefinition, utilizing technology-based formative assessment to transform the learning process of mathematics classrooms into an instructionally modified, augmented or redefined experience, is an area district administrators would describe as requiring further investment. District administrator A stated, "While we know...the trend data show that math continues to be our struggle area... as a school district, it is using these formative assessment tools...that's going to move the needle on the math performance, in addition to content professional development."

Responses from specialist and teacher participants conveyed that in an ideal environment, technology and technology-based formative assessment tools would enhance or redefine learning experiences. Leader B explained,

So if you picture the shallow end (of the pool) as the $\mathrm{S}$ in SAMR and the deep end as the $\mathrm{R}$, we encourage teachers, at various times if you're just going to spend an hour in a pool, you're going to be at various places in the pool at any given time. That being the case, Teacher A stated, "I'm not going to necessarily just give them technology just for the sake of giving it to them." Specialist A elaborated, Mostly, while we would hope that teachers would plunge into the deeper end of the SAMR pool, I would say that most of the time (in math), technology is used as a substitution.... If this question was written this way on paper, I want it to be done the exact same way on a computer, so really used at the substitution level. 
dissertation in practice, B. Jacobs

As noted in previous sections, teachers across sites provided a range of responses on how technology-based formative assessment is utilized to gather and analyze feedback. As a result, data indicated teacher participants emphasized the importance of technology and technology-based tools when it specifically benefits learning.

Overwhelmingly, teacher participant responses indicated the utilization of technology to gather and analyze feedback is beneficial to modification or augmentation of learning experiences. Teacher $\mathrm{H}$ explained the learning is more interactive because, "when we do our target tickets ... or even our quizzes, where they used to just simply fill in the blank with a piece of paper and a pencil, now we can highlight the text, drag and drop." Teacher A stated, "I think that the way IXL gives them their feedback immediately and gives them the description of what they did wrong or how to do it is a good augmentation that enhances that over a paper and pencil."

Conversely, teachers further explained that technology should be utilized when appropriate to the learning standard, as there are process-based standards where substitution, or not using technology at all, is the most effective way for them to gather feedback specific to student learning. Teacher F illustrated, "Technology is great when you use it when it fits. When you try and force it into something that it doesn't really work for it can be cumbersome and very time-consuming." Furthermore, teachers expanded on the use of non-technology based tools when assessing student learning on process-based standards. Many teachers outlined this philosophy, as explained by Teacher H, "As a math teacher, I like to utilize both technology when it's useful and when it's relevant. But a lot of times, we still resort to formative assessment with whiteboards, paper-pencil, (and) working groups." Additionally, Teacher L stated, "I 
would say the learning targets that are processed-based, those are definitely the ones that are harder to assess using technology." With respect to redefinition of learning experiences, teacher participants consistently provided evidence of how difficult it would be to redefine learning experiences in mathematics at this level. Teacher M illustrated, "I think redefinition for us is really difficult to do because we have a curriculum that is jampacked that we can't get through as it is." Teacher C confirmed, "I can't think of anything that we do that would really fall under that. I'm kind of limited to the topics that we're trying to teach in the way we're trying to teach it."

Evidence provided conveyed the sentiments of teacher participants with reference to technology-based formative assessment tools and applications. Statements by teachers confirmed they believe technology-based formative assessment tools impacted instruction and learning. However, the choice to embed technology within the learning process was dependent on the topic and the tools ability to expand learning opportunities in an efficient and effective manner for teachers and students. Further, technology-based formative assessment tools at times modify or augment teaching and learning in mathematics, while it has proven very hard to reach the redefinition stage. Teacher F explained, "I think for math that it kind of falls in that category a lot because math is a very process-based subject and you want to see their process and that requires seeing their work and math is hard to type"

The second data set of technology-based formative assessment that, according to participants, augments learning experiences is student access to instruction and support outside of the classroom utilizing technology-based formative assessment tools. One example is utilizing technology to provide outside learning opportunities, or develop 
prior knowledge utilizing a Flipped classroom approach. Specialist B illustrated, "I think that use the flipped model with fidelity truly does impact student learning, because then the time that's been in the classroom can really be that true feedback and interaction with the person versus the technology." Teacher Participant 3 stated the benefits of students utilizing a flipped model are being able to provide, "videos for the students to watch (at home), and the majority of his practice that they do in class the next day is through IXLs." This evidence suggested a modification occurred which allowed participants to adjust the way information about student learning is gathered and analyzed for real-time adjustment to learning experiences.

Another element, which emerged during data collection, was technology-based tools and applications increase student access to feedback on learning. Principal, specialist, and teacher participants all referenced the ability for students to obtain instant feedback on learning objectives as a benefit of technology-based applications. Principal B stated, "... students can utilize that feedback in and outside of the classroom based on the depth that the teacher gives them." Principal C expanded, "Making the learning more accessible outside of the regular school day by having the devices, and then the learner management system Schoology allows kids access to resources in a centralized location." Principal A communicated one important benefit is

Kids can actually be doing practice outside of school and see what their progress is and be able to have it kind of an immediate tool to show them what they did wrong and what they need to do to correct it

Teacher and specialist participants also conveyed the importance of students having access to applications outside of the classroom that support learning with instant feedback 
and embedded tutorials for guidance. Teacher N stated, "I use IXL and Alex again as their opportunities outside the classroom most often.... it provides explanations and has videos they can watch to improve." Specialist C agreed,

It allows for some of that feedback-based remediation to take place in the absence of the teacher, so students at home, they get a problem wrong, they can not only see that they miss that problem, but then go back and be able to do a little more practice on that concept, whereas that wasn't possible necessarily prior to technology.

The culminating view of all participants indicated technology could augment and modify student learning in mathematics when teachers encourage access and use of technologybased tools outside of the classroom setting.

\section{Using Data Sets}

As previously referenced, district leaders, specialists, and principals hold the expectation that formative assessment feedback is utilized to inform teaching and learning experiences. In addition, each of the building principals, as illustrated in Table 6, communicated the expectation that feedback and formative assessment should be observed daily within each instructional period, and for some corrective action to take place. Although this common expectation existed, when administrators, specialists and teachers, referenced the learning cycle no participant communicated a consistent set of standards for selecting what tools are used to collect data, what data is analyzed, or how data is used to adjust instruction. Teachers were given autonomy to decide which methods they felt best suited their learning environment, which left a noticeable gap in the use common data to guide instruction. The scope of how technology-based formative 
assessment tools and applications impacted data collection, feedback, the adjustment of instruction, and instructional learning opportunities for students varied by site. Provided in this section is evidence provided by participants regarding the impact of technologybased formative assessment tools and applications on how teachers utilize data.

Throughout interviews and focus groups, administrators and specialists outlined an expectation that teachers utilize information from formative assessment to adjust learning opportunities. When considering the impact on the learning cycle, the skill set of teachers and students using technology-based formative assessment tools was a determining factor of whether data collected was used to make a substantial adjustment to the learning cycle. Evidence provided by all six curriculum, instructional, and technology specialists indicated a common belief that most teachers are not equipped with consistent skills and training to implement technology-based methods to their fullest capacity. As a result, specialists concluded teachers miss opportunities to adjust teaching and learning experiences based on a lack of common assessment and data points. Specialist B stated, "technology has really allowed a shift...there is a mindset that because the frequency's there, the access is there, that kids should have that feedback coming at them. And what I see is that teachers do less intervention, less re-teaching”. Specialist E added, "I know for a fact we have teachers who don't know how to drill down on...skill(s) to figure out well, 'Where do I even start with the student?"' Conversely, each district-level and principal participant indicated that within classrooms where teachers were trained on how to utilize technology-based formative assessment, the tools and applications more frequently enhanced the learning cycle to the 
point of augmentation or modification. Specialist B emphasized the role of instructional specialists,

...is to ensure that teachers understand what the learning goal is, and then based off of that decide if using technology in some way is the best way to achieve that goal... That way the technology is really being used as a really added benefit versus replacement when maybe something else would work just as effectively. Principal participants communicated technology-based formative assessment tools allow for a correction to the instructional plan within their learning cycle. Thus, there are times when principal participants observed teachers utilizing technology-based formative assessment tools to provide supplemental learning opportunities otherwise non-existent without technology.

For example, Principal C noted, "I would say more often than not what I see is (technology) enhancing the learning or the instruction. Taking a lesson and being able to go a little bit further with it using technology... (and) able to better personalize the learning for kids.” Principal B confirmed, “... a lot of teachers still use it as substitution. Those who are more comfortable will get into an augmented or an entirely redefinition of something." The instructional sequence is adjusted based on feedback, Principal C notes, as evidenced by the comment, "I've seen teachers realize midstream (when assessing student progress)...Y You guys have already got this. We can move past that." Principal B concurred, “... when a teacher gets that feedback from using those (technology-based) assessments, I think if they are reflective, it could potentially change the course or the line of learning that they may have had lined out. Or it may actually go ahead and 
dissertation in practice, B. Jacobs

confirm that what they've been doing is correct because they've gotten good formative feedback from kids."

Conversely, principal and specialist respondents communicated no measurable or observable difference in classrooms where teachers were not trained to do so. Specialist B stated, "I'm not sure that we're responsive and that we're planning for instruction based off of evidence." Additionally, Principal C stated, "putting a device in a teacher's hand without training is a waste of time." Principal C later stated,

So if I don't know that certain things exist or have the confidence to utilize those in my classroom because I have not learned it, I've not been shown then I'm not probably going to do it on my own and go explore for all of these things that I'm not comfortable with to begin with.

As such, the utilization of data collected from technology-based formative assessment tools and applications, as stated by participants, yielded more productive and meaningful learning experiences when teachers had specific training.

Although district-level leaders, specialists, and principals indicated teacher training as a primary indicator of success, they further communicated training on infusing technology-based formative assessment alone will not yield the desired results. Principal A elaborated, "We've done a huge focus in the last two years on formative assessment in our building, and we have done a lot of large group PD focus[ed] on technology" along with "periodic professional development... bringing our technology specialist over." Specialist B stated,

Formative assessment has been one of our high priorities. I feel like over the past few years we've done such a great job of filling teacher's toolboxes with formative 
assessment strategies. However...when working with teachers, more often than not, I see a lack of understanding of what to do with the assessment data that's collected, and then after processing it, actually using it to impact instruction.

Further, responses by all participants supported this conclusion, indicating over a dozen different technology-based tools utilized by teachers across the district within middle school mathematics. Specialist C further stated, "I think part of the equation that we're missing... is the ability to personalize lessons using technology." Specialist D said, "What ends up happening is they're getting the same lesson more than once that they're getting in the regular classroom." As such, it is clear that district level administrators, principals and support specialists identified the need within the district to infuse purposefully technology-based formative assessment tools with the intention of personalizing, or adjusting, learning experiences based on the information gained by using the tools.

As indicated by the large number of tools utilized by teachers, leader, principal and specialist participants agreed teachers are saturated with a variety of technologybased formative assessment tools. Although a vast selection of technology-based applications existed, only three of them were supported (purchased) by the district, which were ALEKS, IXL and Schoology. In conjunction, teachers had complete autonomy when deciding how, when or if the district supported tools were incorporated within their instructional time. Leader B explained,

Formative assessment is largely left to teacher choice. Do we have a formative assessment tool that we send to every elementary teacher, or for that matter every 
secondary teacher, and say, 'You have to use this for formative assessment?' No.

We don't. It's largely teacher choice."

It is because of this autonomy that a variety of approaches to implementing technologybased formative assessment existed. As a further result, the three applications supported by the district were not utilized in the same fashion within any site, or across the three sites, with the same fidelity.

Principal, specialist and teacher participants indicated the strength of technologybased formative assessment, and an area often lacking in implementation, is the increased access to data. Furthermore, in conjunction with teacher training on the utilization of data, administrators and specialists contended technology-based formative assessment tools would yield more useful and meaningful data if specific applications were implemented more consistently within and across sites. As such, principals and specialists communicated how the lack of specific, consistent, technology-based platforms within district sites resulted in little to no common data points when making decisions in which applications best support student learning. For example, Principal A submitted, "One of the downfalls of the middle school level versus the high school level in our district at least, is that we don't have as much access to data for nearly every department like EOC's." Administrative participants expanded on the topic, indicating without comparable data on the effect of instruction, the math teams within buildings and across the district miss valuable opportunities to impact instructional practice and student learning. Principal B continued, if data were available and consistent across the building, "we would look at that data as a team to discuss maybe if a student needs an intervention class or needs additional support." Principal C agreed, stating, "in the future, when there 
is more consistent common data that the expectation is that teachers look at that together and they...make decisions about how what's happening in their classroom compares to others in their same department." In addition, specific training for teachers on how to utilize common data points within teacher collaboration was a necessary, but missing, element. Specialist C expanded, "Whenever you have the feedback that is provided through the programs, the software, teachers spend time talking about instructional practices to reach those students who aren't understanding concepts or how to manage curriculum instruction in the classroom based on the data that comes in."

To illustrate, data collected indicated that of the 15 teachers within the study, 13 of them referenced utilizing data from formative assessment regularly within the instructional process. Of those responses, participants provided a variety of individualized responses to how each utilized the same applications to review different data points in isolation. For example in Schoology, Teacher H explained, "I can tie in things that they have to do in Schoology, assessments and things, so that even if I wanted to if I wasn't there, I could actually see how they're progressing while I'm not there even before I return to the classroom." While Teacher F stated, "I do like using the Schoology to administer the short assessments." However, Schoology, as described by Specialist F, served as a collection of learning standards, useful only if teachers link questions to the specific standards being measured. Specialist F illustrated, "Schoology in itself, there's nothing there. So unless teachers have created something, unless there's some assessments, things that have been pushed and available to them, Schoology doesn't have anything." Examples of how responses varied by site and teacher regarding the same application were evident with all applications, including IXL and ALEKS. For example, 
in ALEKS, teacher participants in Sites B and C utilize ALEKS for a standing homework assignment for remediation and enrichment. However, each teacher at those sites selected to limit analysis to the number of completed lessons within a given timeframe.

In the absence of a common technology-based platform, teacher participants also noted the lack of common data available for collaboration for improving instruction and learning. Teacher M noted, "In the absence of common assessments, right now a lot of the analysis of any classroom data is done by the classroom teacher alone." Specialist D confirmed, "We don't have a common assessment for them...right now, the commonality between schools and the data is just not there, so there's not much for that piece." All 3 principals referenced a lack of comparable data to analyze, as illustrated by Principal C, In the future, when there is more consistent common data that the expectation is that teachers look at that together and they not only make decisions about how what's happening in their classroom compares to others in their same department, but as a whole, as a department, where are we, as a group, in moving all of our kids towards mastery of the objectives that we have in our class, so that it becomes much more about moving every student than it does just the kids in my classroom. Principal A added, "We don't have as much access to (common) data for nearly every department.... So I think that's much more difficult to do especially since we do not have currently any summative assessments even for our department." Although it was evident through this study that technology provided data for teachers to analyze, it is clear the amount of data gathered and the way in which it was analyzed was dependent on the skill set and training of the teacher. 
dissertation in practice, B. Jacobs

As previously noted, responses by teacher participants suggested multiple methods for how data was gathered and analyzed utilizing technology-based tools and applications. Evidence indicated the variance in how tools and applications are utilized was significantly impacted by the teachers knowledge or skill set with specific programs. For example, Teacher A indicated the use of a variety of reports utilized using applications like IXL and ALEKS to view student growth and progress, while Teachers B and $\mathrm{D}$ within the same site did not know the reports were available. Furthermore, although all 15 of the teacher participants agreed that applications like IXL provided immediate, real-time, feedback to students with example tutorials, some teachers stated this could be a disadvantage. Teacher H explained, "My struggle with some of them, especially IXL, is that the explanations don't always mirror the way that we've taught it. And so then that sometimes confuses the kids." Teacher J expanded, "As a teacher, also feel like I learn more from seeing what mistakes those kids are making" stating there are times when students "rush a lot with any type of technology", which sometimes presents a false set of information.

Despite potential for negative effect, teachers at all sites indicated IXL provided students feedback with respect to growth on specific standards. Teacher J explained, "IXL is really good for if you're looking for something (a standard) specific just because it's easier to navigate. It's real easy just to go log in and type in a couple keywords and search and find something." Teachers A, C, and D at indicated they used that information from IXL on a daily basis as homework to access an electronic glimpse on progress that they use to adjust instruction. For example, Teacher A illustrated, "with the IXL scores, because when you go into IXL it actually has levels... So I can actually go in 
on day two of a skill and group kids (based on level)." Teacher A further elaborated, "I've actually had different assignments, a little bit of differentiation of what they need to do based on those IXL levels and where I put them.” Teacher I used IXL similarly, Specifically with IXL, they have a student center and it shows you what they're working on and if they're progressing at kind of a high level or if they're struggling or they're just kind of in the middle. I typically use that to gauge which students I need to walk around and help. Although formative assessment is a common and expected practice within classrooms across all sites, there does not exist a common and consistent implementation plan regarding technology-based formative assessment. District specialists attempted to accomplish the goal of common tools when they provided a webbased platform with technology-based formative assessment applications for teacher and student access. Specialist F explained, "We have the tools there. They have a lot of tools, almost overwhelming of tools, and then what?" Specialist A concluded, "Teachers need to look at how are my students performing, what do they know, what do they not know and those are the items that need to be retaught or reemphasized or just refocused on." Specialist B illustrated "So I think we could see even greater impacts if we moved away from filling the toolboxes, if you will, to more of the processing of the data, and then really that idea of instructional response." 
dissertation in practice, B. Jacobs

\section{Research Question Two}

How does the professional development and training of teachers and administrators specific to technology-based tools and applications (IXL, ALEKS, Schoology, or other applications) impact the way teachers adapt instructional learning opportunities?

When the outcome of specific applications were examined within this research, the researcher discovered a vast number of technology-based applications being utilized by teachers across sites. In addition to district supported technology-based formative assessment applications like IXL, ALEKS, Schoology, and Google applications, the researcher found eight additional known applications that are utilized within the instructional cycle of teacher participants. Furthermore, although IXL, ALEKS and Schoology were applications being utilized by participants throughout the study, the researcher discovered each were being utilized in a different manner and frequency across sites throughout the district. Concurrently, none of the technology-based formative assessment resources was consistently used to collect common data points. Therefore, the impact of each application on the scope and sequence of instruction, and ultimately the impact on student learning, was situational within each classroom. As such, results of utilizing each application was dependent on the teachers training in using the application for instruction, as well as their knowledge of how to utilize information available within the applications reports and feedback. Evidence to support this came in an observation of teacher collaboration at Site $\mathrm{C}$ where teacher participants were discussing uses of the IXL and ALEKS applications. As a participant was explaining data they were reviewing to adjust upcoming lessons, members of the group communicated a 
lack of knowledge related to how to use the applications to gather the specific feedback being utilized to inform the decision. Although this research discovered a lack of consistency and fidelity with regard to how applications were being utilized, each teacher participant provided evidence to support the conclusion that the use of these applications impact student learning within their classrooms.

Within each site, there existed a level of common implementation with regard to the manner and frequency of how technology-based applications were being utilized. For example, Site A teachers at the $8^{\text {th }}$ grade level all stated they utilize IXL as a homework supplement consistently and with common frequency. Site $\mathrm{C}$ teachers utilize IXL as a method for review at the end of an instructional cycle. With regard to ALEKS, teacher participants across all sites utilize ALEKS as an enrichment tool; requiring students complete a certain number of topics and lessons within a given timeframe. Although each teacher within and across sites utilized these tools on some basis and have access to similar data points from these applications, they each utilize information and feedback from these data points in different ways and with different frequency. The common theme amongst teacher participants was that they all are attempting to achieve the same goals of enhancing, or remediating, student learning with technology-based tools and formative assessment feedback. Further, all participants concluded there are specific ways in which technology-based formative feedback increases student learning, but there are specific times when technology applications or resources are not the preferred method of instruction or measurement. Although there was no clearly outlined plan for how technology-based applications were utilized, the researcher will communicate findings relative to each application. 


\section{IXL for Technology-Based Formative Assessment}

IXL is a district supported technology-based tool intended to provide structured and specific information related to student progress on learning targets aligned with district and state learning standards. All teachers were initially trained when the application was purchased, although training for teachers hired since has not been done

consistently. Further, the depth of knowledge in how this application is utilized varied by participant and was dependent on how much time the teacher had invested in exploring the functions of the application. The researcher found teachers at each site utilized the IXL application in a variety of ways. For example, in Site A, teachers utilize IXL for homework assignments, using daily learning targets to determine which lessons within the program they assign to students for outside completion. Teachers within Site A expect students to work through modules each night as homework assignments and utilize the specific feedback functions to guide them to proficiency. Site B utilizes IXL as a supplemental practice one to two times per week so students get additional practice related to harder topics covered. Teacher participants from Site C utilize IXL as a review for summative assessments, with the intent that students will utilize immediate feedback feature to review and practice learning targets prior to assessment.

Participants within the study, as noted in Table 7, provided evidence to support a conclusion that the tools within IXL benefit the instructional cycle, ability to gather and analyze data, and ultimately that it enhances student learning. Specifically, IXL provides automatic responses to students when they complete a problem, differentiates learning targets embedded within the program, as allows teachers to view reports on student progress and understanding. Teacher A stated one of the benefits to IXL, as opposed to 
traditional assignments is that, "if you're doing in IXL and you get the first one wrong, it tells you immediately."

Within IXL, Teacher I communicated, "They have a student center and it shows you what they're working on and if they're progressing at kind of a high level or if they're struggling. Or they're just kind of in the middle. I typically use that to gauge which students I need to walk around and help.” Teacher C explained IXL would allow teachers to dig into scores to get, "a pretty good overview of how they did on the assignment. It also tells you how many problems they did, how many they missed, how many they got right". Ultimately, all teachers like the format of IXL as it provides immediate feedback to students, as well as information available to teachers through reports. 
Table 7

Impact of IXL on Learning Cycle, Feedback, and Student Learning - Focus Groups and Interviews - Illustrative Quotes

\begin{tabular}{|c|c|c|}
\hline Impact on Learning Cycle & $\begin{array}{c}\text { Impact on Available } \\
\text { Feedback }\end{array}$ & $\begin{array}{l}\text { Impact on Student } \\
\text { Learning }\end{array}$ \\
\hline $\begin{array}{l}\text { Principal B - "those that have } \\
\text { specific objectives that are tied } \\
\text { to progression" } \\
\text { Teacher H - "My struggle } \\
\text { with...IXL, is that the } \\
\text { explanations don't always } \\
\text { mirror the way that we've taught } \\
\text { it. And so then that sometimes } \\
\text { confuses the kids. So I just have } \\
\text { to work them out ahead of time } \\
\text { to find that out" } \\
\text { Specialist C - "A lot of the } \\
\text { teachers use IXL as their } \\
\text { homework assignments so you } \\
\text { can assign based on what the } \\
\text { standard is and gives them a } \\
\text { little bit more practice" } \\
\text { Principal A - "So you could } \\
\text { potentially have one teacher } \\
\text { using IXL very efficiently for } \\
\text { student benefit and another } \\
\text { teacher hardly using it all" } \\
\text { Specialist E - "I would also add } \\
\text { teachers are looking for } \\
\text { computer graded (programs) } \\
\text { like IXL" }\end{array}$ & $\begin{array}{l}\text { Teacher A - "(I like) IXL for } \\
\text { that immediate feedback that } \\
\text { they get toward-- it's helpful } \\
\text { for me as a teacher" } \\
\text { Teacher C - "IXL will let you } \\
\text { dig down however much you } \\
\text { want to know...Their scores } \\
\text { are what they call a } \\
\text { SmartScore...If you get an } \\
\text { 80, it means that you are } \\
\text { proficient at a topic" } \\
\text { Teacher D - "So I can look at } \\
\text { those scores, and I can look at } \\
\text { the time students spent, and it } \\
\text { gives me an idea of what they } \\
\text { did. I can drill into it" } \\
\text { Teacher F - "I think you can } \\
\text { get feedback pretty quick } \\
\text { from students when you } \\
\text { administer an assessment } \\
\text { electronically, and the } \\
\text { students can get the feedback } \\
\text { fast as well because they get } \\
\text { it instantly whenever they } \\
\text { finish" } \\
\text { Leader B - "We're able to } \\
\text { very quickly-- large groups of } \\
\text { students not mastering a } \\
\text { target so the teacher can } \\
\text { quickly go back and revisit } \\
\text { that topic" }\end{array}$ & $\begin{array}{l}\text { Teacher E - "If they're } \\
\text { doing a worksheet and they } \\
\text { do } 10 \text { problems, they don't } \\
\text { find out tomorrow and they } \\
\text { did them wrong. Whereas } \\
\text { if you're doing in IXL and } \\
\text { you get the first one wrong, } \\
\text { it tells you immediately" } \\
\text { Teacher B - "I think that } \\
\text { the way IXL gives them } \\
\text { their feedback immediately } \\
\text { and gives them the } \\
\text { description of what they } \\
\text { did wrong or how to do it is } \\
\text { a good augmentation that } \\
\text { enhances that over a paper } \\
\text { and pencil" } \\
\text { Principal A - "IXL...for } \\
\text { formative assessment has } \\
\text { had a positive impact } \\
\text { specifically because those, } \\
\text { I think ,more closely mirror } \\
\text { the testing environment" } \\
\text { Teacher N - "IXL is really } \\
\text { good for them to review } \\
\text { specific concepts if they } \\
\text { need to practice for a test or } \\
\text { if there's a concept they're } \\
\text { struggling with, they can } \\
\text { go in and review that very } \\
\text { specific concept...(as well } \\
\text { as) it's just a good resource } \\
\text { to let them practice on their } \\
\text { own" }\end{array}$ \\
\hline
\end{tabular}


dissertation in practice, B. Jacobs

\section{ALEKS for Technology-Based Formative Assessment}

ALEKS is a technology-based application supported by the district, and utilized across sites as a supplement to daily learning experiences. The fidelity of usage varied across sites, however, the researcher found some commonalities within each site with regard to the purpose and frequency of use. As previously sated, ALEKS is consistently utilized across sites as a supplement to instruction. Teacher F explained, "All of our students also have access to ALEKS, a web-based artificial intelligence assessment and learning system to fill knowledge gaps and to enhance course content." According to Teacher $\mathrm{J}$, the

ALEKS program also allows our students that are more advanced to be challenged at the level that they are currently working on in that program... it addresses whatever concepts that may be currently missing and helps them work on building maybe more skills from prior grade levels, and helps them learn those through technology, that maybe we can't address in our classrooms.

In Sites B and C, teachers require students to complete a predetermined number of topics within a unit or timeframe. In this process, students have the autonomy to choose which topics they will complete, when they will complete them, and how long they spend reflecting on feedback provided by the application. Therefore, the effect of ALEKS on daily instructional practice is minimal unless a student coincidentally completed a topic prior to being covered in class. Teacher participants across sites explained there are times when students enter with prior knowledge due to the completion of topics in ALEKS. Teacher F stated, and multiple participants agreed, "I'm sure we've all heard multiple times through the year. We'll introduce something new and a student will say, 
'Oh. I did that in ALEKS.' So it kind of builds a good base for them sometimes for the new topics or concepts that we haven't gotten to yet."

As reflected in Table 8, although ALEKS has a limited impact on daily instruction, it clearly impacts the learning cycle for students within learning standard explored to personalize, differentiate and provide structured feedback that informs learning. ALEKS does provide access to rich and meaningful data to students which teachers can utilize the program to obtain data specific to each learning standard attempted. Although teachers across this research do not consistently access and utilize data from these programs, participants noted immediate and personalized feedback students receive from this technology-based formative assessment application allows students to reach a deeper level of understanding in a more efficient manner.

\section{Schoology for Technology-Based Formative Assessment}

While Schoology has been selected as the districts learning management system, implementation of the program district wide varied by site and participant. Ultimately, district leaders indicated the method of immersion for Schoology is to train staff members on a voluntary basis on the capability of the program, link tools for instruction and assessment, and provide a common technology-based platform to manage student learning. When choosing Schoology, Leader B indicated the district considered factors such as, "Does it align with state standards... is it teacher friendly... student friendly? Can we get the data out of it that we need?" The vision of the district is to provide consistency within access, training, and utilization of technology-based formative assessment tools. 
Table 8

Impact of ALEKS on Learning Cycle, Feedback, and Student Learning - Focus Groups and Interviews - Illustrative Quotes

Impact on Learning Cycle Impact on Available Data

Teacher C - "ALEKS is used as additional.... So it's more of a reinforcement of things in learning. It does not tie to what we're learning in class"

Specialist C - "ALEKS is where remediation is offered and it takes kids... back to the point of misunderstanding"

Teacher L - "ALEKS are used probably the most frequently to help them review and also to differentiate learning."

Specialist D - "ALEKS is great for customizing to the student, and it can give them below grade-level interventions or extend their knowledge if they're past grade level" and get really, really detailed
Teacher B - "ALEKS, those that have specific objectives that are tied to progression, I think that's probably honestly more of a summative look for teachers."

Teacher D - "So ALEKS I think does as far as detailed information-- well, you can really dig down into ALEKS information... they have immediate feedback after every problem"

Teacher $\mathrm{H}$ - "We can see with...ALEKS exactly how they answer the questions so we can go back in and correct their mistakes"

Leader A - "ALEKS... give(s) very specific objective reports back to teachers.... you can watch what happens on certain learning standards"
Impact on Student Learning

Teacher A - "And so teachers perhaps may spend less instruction time one-on-one with their class, but their modeling time is more specific."

Teacher D - "Alex, they have immediate feedback after every problem"

Leader A - Objective reports, "then help students set goals and help parents understand what the goals are for those students.

Principal A - "Students work "outside of school and see what their progress... and have an immediate tool (to show what) they might be missing that they need to improve upon before they can really master that particular skill"

\section{Leader A confirmed,}

We're working to build banks for teachers that they can all utilize with some consistency relative to formative assessment tools, but we haven't dictated or mandated use of specific formative assessment tools in any content area or at any level. 
Although the responses by participants indicated, as noted in Table 9, Schoology

positively impacts the learning cycle, data available to teachers and students and

ultimately student learning, the triangulated evidence given to support those claims varied in great fashion across sites and in comparison to district level responses. As such, the data in this section will provide evidence to support a positive effect by Schoology on student learning, but for different reasons depending on the given site.

\section{Schoology in District}

Within the district, it is imperative to provide resources to support student learning. Seeking out opportunities to identify the technology-based tools most likely to promote learning opportunities is a process leaders take seriously. Once technologybased tools aligned to district curriculum and philosophy are identified, Leader A explained it is then necessary to "make those (tools) accessible through (specialists) websites, the assessment office, and curriculum specialists" and train teachers to utilize them. Leader A elaborated,

If we're going to support learning of all students, that we have to monitor them constantly all the time to make sure no one is falling off the radar, if you will, and that our schools at all levels have appropriate supports for those kiddos.

Although the vetting process for technology-based tools is clearly outlined, the district lacks a system for identifying specific tools to be commonly utilized across sites. Leader B illustrated, "Do we have a formative assessment tool that we send to every elementary teacher, or for that matter every secondary teacher, and say, 'You have to use this for formative assessment'? No." Therefore, Schoology was implemented on a voluntary basis across district sites, and thus, encouraged teachers and sites to participate in training 
Table 9

Impact of Schoology on Learning Cycle, Feedback, and Student Learning - Focus Groups and Interviews - Illustrative Quotes

\begin{tabular}{|c|c|c|}
\hline Impact on Learning Cycle & Impact on Available Data & $\begin{array}{l}\text { Impact on Student } \\
\text { Learning }\end{array}$ \\
\hline $\begin{array}{l}\text { Teacher H - "In terms of my } \\
\text { planning and instruction, I } \\
\text { guess when I'm creating maybe } \\
\text { something on my own in } \\
\text { Schoology....It has challenged } \\
\text { me to think about how to ask } \\
\text { questions in a different way" } \\
\text { Specialist F - "Schoology has } \\
\text { Conference, which is similar to } \\
\text { Skype and a Google hangout, } \\
\text { but it's just for that class of } \\
\text { students, so students are } \\
\text { studying for an exam, or a } \\
\text { topic's new... kids don't get off } \\
\text { track" } \\
\text { Teacher L - "Schoology on the } \\
\text { Chromebook just as a method to } \\
\text { provide notes for them if they're } \\
\text { not there, and a blank copy of } \\
\text { what we did that day if they } \\
\text { were absent" } \\
\text { Teacher D - "with Schoology } \\
\text { we can look at the assignments } \\
\text { and tell how they did, to } \\
\text { see... where we need to go. }\end{array}$ & $\begin{array}{l}\text { Teacher H - "Most of the } \\
\text { assessments I create, I've } \\
\text { been trying to tie and tag the } \\
\text { learning objective on there. } \\
\text { So the kids can't see it, but } \\
\text { from a teacher perspective, I } \\
\text { could go back in through and } \\
\text { sort the data" } \\
\text { Teacher G - "if I push a quiz } \\
\text { or an assessment out through } \\
\text { Schoology, the data points go } \\
\text { in onto the kid's IEP goal." } \\
\text { Leader B - "There's a } \\
\text { mastery view that I know } \\
\text { some teachers are using, } \\
\text { broken down by standard" } \\
\text { Specialist D - With } \\
\text { schoology, I used it in the } \\
\text { classroom for formative } \\
\text { assessment with a three to } \\
\text { five question daily. And in } \\
\text { the way that you could grade } \\
\text { it, you just go through by } \\
\text { question, and you could see } \\
\text { which questions kids were } \\
\text { struggling on. And then you } \\
\text { would have quick feedback } \\
\text { every day on who was } \\
\text { understanding, who wasn't } \\
\text { understanding, and then you } \\
\text { could pull small groups. }\end{array}$ & $\begin{array}{l}\text { Principal C - "Schoology } \\
\text { allowing kids access to } \\
\text { resources in a centralized } \\
\text { location that had been } \\
\text { vetted already by teachers } \\
\text { to be appropriate for kids" } \\
\text { Teacher A - "I have in } \\
\text { Schoology a portfolio that } \\
\text { the kids do where they } \\
\text { keep all of their } \\
\text { assignments. And so, they } \\
\text { have constant access to } \\
\text { that...and assignments that } \\
\text { we've written, that gives } \\
\text { them automatic feedback" } \\
\text { Specialist D - "the only } \\
\text { way in Schoology a student } \\
\text { would see that is if learning } \\
\text { targets are attached to that, } \\
\text { and then they can go to } \\
\text { mastery which is actually, } \\
\text { to me, more informative for } \\
\text { teachers and students than } \\
\text { looking at a grade" }\end{array}$ \\
\hline
\end{tabular}

by providing funds, while hoping increased access and the capabilities of the tool would

be compelling enough for teachers to choose the platform. Leader B explained that three 
years into implementation, "We have three- quarters of our teachers using the Schoology learning management system on a daily basis. And so that's a big growth. And honestly, that's all been done through optional trainings."

The difference between Schoology and the technology-based tools specific to mathematics is Schoology encompasses learning targets across all content areas. Furthermore, Schoology as a system has not created tools for measuring learning, but a system for storing curriculum, assessments, and instructional tools, all of which are created or inserted by district staff members. Once assignments, assessments or projects are created and, "when you do link learning targets to anything they do, they (teachers and students) will have mastery data on those pieces," stated Specialist F. Schoology also added an element of access for parents and students to find resources and supports to learning. So, the district choice to adopt a management system was communicated as a large scale approach to monitoring student learning, and providing consistent training to all teachers.

\section{Schoology within Sites}

As previously stated, each site utilized Schoology in a differing ways and for a variety of purposes. Teacher A was the only participant who stated, "I have in Schoology a portfolio that the kids do where they keep all of their assignments. And so, they have constant access to that." This allowed students to have access previous work and feedback to inform current learning stored in one location. Teacher F utilized to increase assessment opportunities, as illustrated by, Before, really, we would just give a quiz, and maybe that happens once a week or every seven or eight school days, something like that. But now we are taking a quick assessment with target tickets through Schoology, 
and we get feedback back to them very quickly. Principal C, also noted, "The assessments that are being written in Schoology and the new... allow for teachers to get some immediate feedback" and Teacher C confirmed, "With Schoology we can look at the assignments and tell how they (students) did." Both statements supported the fact that feedback through Schoology can enhance learning and allow for adjustments to learning experiences.

Participants from each site provided responses in support of how Schoology can enhance student learning, however, also provided evidence to why they preferred math specific technology-based tools. Where math-specific applications like IXL and ALEKS provide immediate feedback after every question, Teacher participants $\mathrm{B}, \mathrm{D}$, and $\mathrm{F}$ all stated Schoology provides feedback upon completion of the assessment. Teacher D illustrated, "and because our students are so used to the IXLs, I sense frustration personally with Schoology because they (students) don't get that, 'Did I get this one right or wrong before I go on?"' Furthermore, the lack of common resources within Schoology increased the discrepancy amongst sites, and brought an increased level of attention to the quality of training teachers have in creating assessments. When asked if Schoology provides resources for feedback, Principal C stated, "Schoology does not unless they (teachers) built a quiz in Schoology and the students are taking the quiz there (in Schoology) and the teachers have released the information." Therefore, teachers with higher levels of expertise with the tool, in conjunction with increased ability in creating assessments, found the information gained from the program was more beneficial and impactful. Teacher H stated, "Schoology allows us to do the same thing depending on how we set up the assessment. So those are different ways we can use that." Participants 
dissertation in practice, B. Jacobs

from across sites indicated the increased support for Schoology in the form of trainings

and access to technology specialists. As evidenced by teacher comments, the district has attempted to close those gaps that exist in teacher capability with regard to Schoology and formative-assessment creation. Teacher $\mathrm{M}$ illustrated the technology specialists will be, "over here any day as long as it works on his schedule... he's very flexible and will actually sit and build something with you. You tell him what you want, and he's got that time to really work with us."

\section{Research Question Three}

How do technology-based formative assessment resources impact the frequency and quality offeedback utilized by teachers to enhance teaching and learning?

District level administrators communicated the commitment to providing support to teachers through increasing access to technology-based tools, training, and personnel specifically designated to increase instructional efficiency. Leader A stated, "If we're going to support learning of all students, we have to monitor them (students) constantly... and that our schools at all levels have appropriate supports for those kiddos." Leader B expanded on how teachers have access to information that shapes instructional units. Regarding analyzing of progress, Leader B stated,

I think that's the advantage sometimes of using a learning management system. You've got different (non-traditional) views for those grades. So for instance in Schoology, there's a mastery view that I know some teachers are using, broken down by standard.

Teacher F expanded, "Before we would just give a quiz...once a week or every seven or eight school days.... now we are taking a quick assessment with target tickets through 
Schoology, and we get feedback back to them very quickly." As such, all participants contributed evidence to support a claim that monitoring learning regularly is an essential component to student learning. Further, Principal B stated technology-based formative assessment positively impacts student learning, stating, "it increases and enriches the type of feedback that they (students) get.... and the frequency of how they can give feedback and the rate at which they can give it is also increased."

In addition, Teacher $\mathrm{H}$ illustrated, "the good thing about our technology is that we can get very specific, detailed data about where our students are at." In addition, Teacher F stated providing feedback formatively prior to assessment allows teachers and students to make real-time corrections on questions simulating the "same type of quiz-type assessment, just much shorter in length." Specialist C added, "I think when looking at and talking about how teachers provide feedback, I think technology enhances that (process)," and technology-based feedback is communicated "faster for them than handwritten feedback." One benefit noted by Teacher I was the capability within technology-based formative assessment tools to allow teachers to, "see the time spent on the activity. That helps me. If it's taking way longer than it should, then I know that some re-teaching needs to be involved, and I don't get that feedback from paper-pencil." Teacher H illustrated,

One of the biggest impacts I have seen... the timing of the frequency (of feedback). If I give, say, a target ticket, which is an assessment on one learning target, I can usually catch a kid before they walk out the door of the classroom. 
dissertation in practice, B. Jacobs

This allows teachers to make modifications within a shorter period and increase remediation or re-teaching opportunities faster than would be accomplished without technology tools.

Teacher I conveyed, "The immediate feedback is great," and with the availability of immediate feedback, it allows students to correct and resubmit after fixing errors from the initial completion. Leader A identified, "the feedback to students is much more immediate... and pacing for a classroom teacher seems to be much more with the locus of control of the classroom teacher." Leader B further noted,

I think that's where my role and our (technology) specialists come into play....That's a big part of what we do, when we work with teachers, is finding ways in that system (Schoology) to assess kids that's standards-aligned, that can be done frequently, and that, more importantly, can kick some data back to teachers and students really fast.

When the quality of feedback, specific to middle school mathematics courses was considered, responses from teacher participants indicated the learning target, or standard, played a large role in determining if technology would increase the effect on learning. As stated earlier by Teacher $\mathrm{H}$, realizing the time to use technology-based tools is a function of when it fits the situation and the desired learning of students. Teacher J expanded, The way that technology is changing, these kids are going to have access to just right and wrong answers. It's going to be who can apply it, who has the process to make sense of their answers, and who can really reason what the right answers are and how will they use those, and we can't assess that with technology. 
Teacher participants further explained that technology can take teachers too far away from the feedback process, especially when students are monitoring their own progress. Teacher I stated, "Students who get a $60 \%$ are okay with getting a $60 \%$...even with immediate feedback that they're not progressing with a skill, there's still a lack of connection of going back and trying to improve their understanding." Teacher F stated although technology provides more instant access to feedback in mathematics, the quality, or, "Detailed feedback, we're giving some of that up so that we can get the feedback to them faster."

In addition, teacher participants conveyed it is sometimes more efficient and effective for them to gather information on student learning without utilizing technology. Teacher J expressed, "I walk my room every day and look at student homework on paper. And that redirects my lesson.... based on the feedback they give us on individual work, then that determines how we move forward with curriculum.” Teacher $\mathrm{H}$ confirmed, "When they do things on paper, I feel like I actually have a better sense, when I'm grading it, of what the common mistakes are." Teacher E communicated, "So with math, it's usually the more technology we do, the farther away they get from a piece of paper and a pencil, and how beneficial is that when you're doing math?" Teacher A followed up stating, "You still need to do it on paper," and teacher D stated a drawback of technology being, "they are they are disconnecting from just the math part of it", indicating the importance process and application play in learning math. Included in Table 10 are some examples of timeliness and quality of feedback 
Table 10

Timeliness and Quality of Feedback of Technology Applications - Illustrative Quotes

\begin{tabular}{|c|c|c|}
\hline Application & Timeliness of Feedback & Quality of Feedback \\
\hline IXL & $\begin{array}{l}\text { "Benefits in both timeliness and } \\
\text { availability. I mean, it opens up } \\
\text { opportunities outside of the regular } \\
\text { class time, outside of the regular school } \\
\text { day." }\end{array}$ & $\begin{array}{l}\text { "Where remediation is offered and } \\
\text { it takes kids...back to the point of } \\
\text { misunderstanding... online } \\
\text { programs and computers can aid in } \\
\text { that process to personalize learning } \\
\text { for kids" }\end{array}$ \\
\hline ALEKS & $\begin{array}{l}\text { Kids can actually be doing practice } \\
\text { outside of school and see what their } \\
\text { progress is and be able to have it kind } \\
\text { of an immediate tool [inaudible] them } \\
\text { to show them what they did wrong }\end{array}$ & $\begin{array}{l}\text { "it (ALEKS) does offer them a } \\
\text { very detailed report about where } \\
\text { they are on each learning target for } \\
\text { their grade level, and it does give } \\
\text { them feedback at any time that they } \\
\text { choose" }\end{array}$ \\
\hline Schoology & $\begin{array}{l}\text { "Assessments that are being written in } \\
\text { Schoology...allow for teachers to get } \\
\text { some immediate feedback (and teachers } \\
\text { can) set it to release results to all } \\
\text { students so it can be shared whenever } \\
\text { the teacher feels it's appropriate" }\end{array}$ & $\begin{array}{l}\text { You (can) assign students content, } \\
\text { or you teach students content, they } \\
\text { take a quick formative assessment, } \\
\text { and then depending on those } \\
\text { results, the system itself would } \\
\text { assign remediation or enrichment } \\
\text { content based on how they scored } \\
\text { on that assessment }\end{array}$ \\
\hline
\end{tabular}

\section{Discussion of Findings}

This analysis came in the form of emerging themes from interviews of district and site leaders, focus groups with instructional specialist and teacher focus groups, observation of meetings, and analysis of documents from the district. Three emerging reoccurring themes evolved: The impact of mobile learning on mastery learning; The Impact Mobile Learning on the Quality and Timeliness of Data; Lack of fidelity, training and accountability 


\section{Theme: Impact of mobile learning on mastery learning}

Researchers have argued that mobile learning theory utilizes technology to transform learning, creating instant and expansive opportunities within and outside of the classroom for learning to occur (Romrell et. al., 2014). Furthermore, technological tools and applications can be utilized to substitute, augment, modify, or redefine traditional learning activities in a way that maximizes the experiences for effective instructional design (Puentedura, 2013). The data collected from participants, observations, and documents supported an inference which is indicative of the level of technology infusion within the learning cycle is dependent on the purpose for infusion and the effect technology had on the identified learning standard. Participants provided evidence to support an increase in outside learning opportunities for students, such as tutorials, videos, and instant feedback on failed learning standards. Furthermore, principal participants observed the programs primarily utilized by teachers offered expanded learning opportunities when achieving mastery, as well as providing feedback that encouraged remediation when necessary. Similarly, researchers asserted through use of technology-based tools that learners could obtain information in different contexts, thus allowing for a more effective transfer and commitment of information to memory (Hwang \& Chang, 2011; Puentedura, 2013; Romrell et. al., 2014). As such, this research supports mobile learning theory researchers, (Dyson \& Frawley, 2016; Lee, Feldman, \& Beatty, 2012), in promoting the incorporation of technology-based feedback and formative assessment as a part of the instructional process.

Furthermore, according to Dyson, Litchfield, Raban, and Tyler (2009), the interactive systems available within mobile learning de-emphasize traditional teacher- 
centered models and encourage an active learning process. Again, this is evident in these data sets as illustrated by how teacher participants utilized technology-based applications to provide learning experiences to students outside of the school day. Teacher participants identified remediation and self-monitored learning of specific learning standards outside of instructional time as beneficial to the implemented technology platforms. This supports that effective utilization of mobile devices and applications must consider instructional design when using applications and tools to augment and modify learning experiences. This was evident throughout the data collected, and further supported in the work of researchers (Hwang \& Chang, 2011; Kihoza, Zlotnikova, Bada, \& Kalegele, 2016) who concluded the utilization of technology augments the teaching and learning process by increasing the functionality of formative assessment feedback. Teacher participants within this study, as well as district instructional specialists, asserted technology-based formative assessment tools allowed for learning experiences to occur within, and outside of the instructional space that would not occur otherwise without technology.

Researchers of the SAMR model (Jude et. al., 2014; Puentedura, 2013; Romrell et. al., 2014) asserted the use of technology could enhance and transform learning experiences, directly impact student learning, and achievement when implemented beyond the substitution level. Moreover, analyzing the results of mobile learning activities to increase the quality and regularity of formative feedback to students will maximize the effect of MLT activities on student learning (Cochrane, 2012; Wong, 2016). Furthermore, Dyson and Frawley (2016) stated the implementation of MLT 
dramatically improves formative assessment feedback and marks, as supported by the results of this inquiry.

When technology-based formative assessment tools are utilized as a substitute for traditional methods, participants within the study communicated the reasoning behind the selection is often because they identified substitution as the best method of addressing the learning standard. Statements by teacher participants consistently referenced the inability of technology-based tools to provide information within certain processes students use to address learning standards. As noted by Teacher F, "math is a very process-based subject...you want to see their process and that requires seeing their work, and math is hard to type." Therefore, augmentation and modification of learning occurred because of the expanded use of technology-based tools outside of the classroom on specific learning standards. According to Duijn et. al. (2017), feedback should come from a credible source, address strengths and improvement areas, provide specific instructions for improvement, be provided immediately and should occur regularly. Data from participants indicated technology-based applications, such as IXL and ALEKS, accomplished each of these qualifying standards when infused with fidelity.

In addition to the characteristics previously communicated, Xie (2016) conveyed the utilization of mobile learning strategies to provide feedback allows students to obtain feedback from multiple locations, contains individualized learning content specific to student response, and occurs in a nearly instantaneously fashion. Data collected from the teachers indicated technology-based formative assessment tools utilized by mobile devices allowed learning to be personalized, transferrable, and differentiated based on student ability. Specifically, data collected from teacher participants indicated the 
traditional practice for students (worksheets) meant you had to give the same type of practice to all students and ask them to complete it in the same timeframe. While, the use of technology-based formative assessment applications allowed students to practice at the level of competency within and outside of the instructional space. Data from all participants further suggested technology-based tools encouraged students to work in the same learning standard until mastery. The triangulated data collected from all sources within this study also indicated the individualization of instantaneous feedback because of technology-based tools positively affected and expanded student learning. All participants, as well as district documents, supported the assertion that utilizing functions within the technology-based tools allowed for an increased opportunity for students to assess their own skills, correct mistakes and revisit specific learning standards.

\section{Theme: Impact of mobile learning on the quality and timeliness of data}

When connecting MLT and formative assessment, Hwang and Chang (2011) explored formative assessment-based mobile learning as an approach for utilizing mobile devices to provide immediate feedback to students during learning activities. Further, Conejo, Garcia-Vinas, Gaston and Barros (2016) employed a web-based tool to incorporate Technology Enhanced Formative Assessment that provided elaborate and immediate feedback, and thus concluded students utilizing the designed tool outperformed those utilizing traditional formative assessment methods. While research on the effects of mobile learning on student achievement are found to be beneficial, conversely Afshari et.al. (2009) asserted teacher ability within technology integration, quality of hardware and software resources, available time for collaboration within staff members, and lack of training and support for teachers within technology infusion are 
barriers to teacher implementation of mobile learning strategies. According to

Ekanayake and Wishart (2014), the variables associated with teacher training and support have not been explored with fidelity within mobile-learning research.

However, responses from all participants emphasized the use of IXL, ALEKS, and Schoology applications provided an increased level of timely feedback to teachers and students. Data from principal participants further supported the assertion that technology-based formative assessment tools provide accurate and immediate feedback to students and teachers which allows for adjustments to learning opportunities. Evidence obtained from district leaders outlined the vision for technology infusion was to provide more information to teachers and students, more frequently, and in a more efficient context in order to maximize student learning. All participants agreed an increase in the frequency and immediacy of feedback had a positive effect on student learning through an increase in specific remediation tools and strategies.

Conversely, data from specialist and teacher respondents indicated immediate feedback would not influence student learning unless students and teachers take advantage of the available resources for adjustment or remediation. Teacher participants indicated the availability of immediate feedback, both within and outside of the instructional time, provided increased learning opportunities for students who were motivated and chose to participate in supplemental learning activities. Teachers, principals and specialist participants provided data to support a positive effect on student learning when teachers utilized information to adjust and adapt learning activities, including the addition of modified, re-teaching opportunities when data suggested a lack of proficiency. Crompton and Burke (2017) asserted the need to provide practitioners 
with a guide for utilizing and effectively implementing mobile learning, reducing the emphasis on student-centered initiatives and influencing student learning through instructional design. Although responses indicated a lack of adjustment by students and teachers could nullify the impact of feedback, participants overwhelmingly supported an assertion that technology-based tools provided immediate feedback on student progress within specific learning standards more frequently, and thus had a positive impact on student learning.

\section{Theme: Lack of fidelity, training, and accountability in utilizing feedback data}

When referencing technology-based formative assessment, utilizing technology augments the teaching and learning process by increasing the functionality of formative assessment feedback, therefore enhancing student learning through reflection, collaboration, real-time adjustment to learning activities (Hwang \& Chang, 2011; Kihoza, Zlotnikova, Bada, \& Kalegele, 2016). Researchers (Heitink, Van der Kleij, Veldkamp, Schildkamp, \& Kippers, 2016) asserted formative assessment should include "substantial, constructive and focused feedback" (p. 50). While Rochelle, Penuel, and Abrahamson (2004) identified four constructs to unify technology-based instruction. Those constructs

are: a) formative assessment, b) driving discussion by important conceptual contrasts, c) shifting to mastery-oriented motivational incentives, and d) harnessing diversity for generativity. Feldman and Capobianco (2008) expanded on the four constructs by further identifying the influence of curriculum integration and incorporating methods for constructing formative assessment items as components relevant to impacting teachers' practice. 
dissertation in practice, B. Jacobs

While one objective communicated by all participants was to increase frequency and timeliness of formative assessment feedback, data from participants revealed a large number of technology-based tools, being utilized inconsistently across sites. District leader and specialist responses provided evidence to support teacher autonomy and choice when determining which technology-based formative assessment tools were utilized, as well as how they were infused. Therefore, responses by participants identified over fifteen technology-based formative assessment applications being utilized within the study. Specialist participants expanded on this topic stating teachers have access to an abundance of technology-based tools, as the district has focused on providing technology-based formative assessment tools to teachers. Because of implementation of technology-based tools being voluntary and autonomous, the factors which impacted the selection of applications and how learning was effected across sites were inconsistent.

Furthermore, in the case when consistent applications were chosen, the way in which they were infused varied across sites and teachers. One example is that some teacher participants utilize IXL to supplement learning activities, while others utilized the program for daily homework and practice. As such, all participants indicated a deficiency in teacher skill sets relative to common technology-based tools. This resulted in teachers not possessing the same skills and receiving training uniformly across sites. Concurrently, responses from all participants indicated a lack of adequate knowledge and training for teachers and students on how to utilize data from technology-based applications with fidelity to augment or modify instruction and learning experiences. This lack of fidelity did not allow for common data points within the process of 
collaboration or professional development. Furthermore, determining whether an application had an effect on student learning was largely dependent on which teacher participant was utilizing the program. Responses from teacher participants at one site regarding the effect of technology-based applications at times contradicted those made at other sites within the district. This was also the case regarding how data was collected, analyzed, and utilized to impact instruction. Ultimately, the variance in participant responses was directly related to the skills, abilities, and training relative to the program. As such, the voluntary nature of utilizing technology-based formative applications, in conjunction the varying levels of teacher competency, supported assertions from participants that technology-based applications have the ability to impact learning more so than is currently being realized.

As noted by district leader and specialist participants, teachers are not there yet regarding their potential for technology-based tool utilization. Leader, specialist, and principal participants conveyed a gap existed between the potential for technology-based tools to impact the learning cycle and what is actually being accomplished. So while formative assessment feedback includes opportunities for clarification of goals, selfreflection and assessment, and collaboration, thus allowing teachers and students to identify and properly perceive gaps in learning, interpret the results, and make adjustments within learning activities (Vaughn et. al., 2013; Wiliam, 2011), teachers have not maximized the possible benefits. Similarly, Nicol and Macfarlane-Dick (2006) and Wiliam (2011) proposed incorporating embedded formative assessment as a means to share teaching practices and provide feedback to students, both of which have a positive impact on student achievement. Within this research, the lack of consistency in which 
applications were utilized, a lack of common data points utilized for collaboration, and a lack of ability by teachers to analyze data to adjust instruction were evident barriers to maximizing student learning.

\section{Conclusions}

While past efforts of providing classrooms with technological tools such as computer workstations and Smart Boards increased the level of technology access, researchers (Jude et al., 2014; Wiliam, 2011) maintained initiatives in technology have failed to increase achievement as such efforts merely modified the method of providing equivalent instruction. Furthermore, researchers asserted that infusing technology in classrooms where teachers are trained to enhance feedback and modify instruction based on information gained should be further studied (Vaughn et al., 2013; Wiliam, 2011). This research sought to provide confirmation that teachers using technology-based formative assessment tools within the instructional cycle will further create conditions that promote student reflection on learning within and outside of the instructional space. Specifically, the purpose of this research was to determine if technology-based formative assessment applications impact student learning on specific learning standards. This study analyzed mobile learning opportunities, specific applications (IXL, ALEKS, and Schoology), and the quality and frequency of feedback to determine whether technologybased formative assessment applications provides data to inform instruction and learning.

Research from this study indicated teacher's utilization of technology-based formative assessment applications builds upon the principles of mobile learning theory, allowing students to receive feedback in a variety of ways not possible without technology. Students who utilize applications for remediation, proficiency and 
enrichment opportunities do so at greater rates, and with increased differentiation, when utilizing technology-based applications. Students obtain feedback in real-time, relative to the learning standard, and with immediate resources for explanation of deficiencies and remediation. Further results from this research conclude when technology-based formative assessment is utilized to augment or modify learning experiences, students have access to more information and learning experiences are personalized based on the students level of proficiency.

This research also showed there is a difference in implementation of technologybased formative assessment across sites. These variances can be attributed to several contributing factors. The first factor contributing to the discrepancy of implementation is the lack of a consistent plan to infuse and embed technology-based formative assessment applications across district sites and classrooms. Although a few applications are supported (purchased) by the district, there are no expectations set forth by leadership that require teachers to use them. The type and frequency of use is an autonomous decision made by each teacher. In addition, even when teachers utilize the same applications, the research showed they do so inconsistently and with different purpose. Despite not having a consistent plan across the district for embedding technology-based formative assessment applications, the research indicated each of the teacher participants do have regularly infused and imbedded technology-based formative assessment within the instructional cycle of their classrooms.

In addition, findings from this research show the infusion of technology-based formative assessment applications allows students to receive feedback on learning more frequently, often times immediately, which allows for remediation or enhancement of 
learning at an increased pace. Prior to technology-based feedback, participants indicated it could take several days for students to receive information regarding mastery of a learning standard. Further, prior technology-based applications, feedback was holistic rather than individualized and confined to context of the classroom setting. Technologybased feedback allows for personalized learning opportunities for students in real-time, with the ability to obtain remediation and assistance on learning targets in a non-teachercentered manner. Students can practice at an individualized level, receiving homework that is individual to them, when previously homework and practice was only differentiated if the teacher possessed the skills and knowledge to create individual homework assignments. Technology-based formative assessment, as evidenced in this research, increases or decreases the level of difficulty based on the current level of student understanding. Mobile learning enhances student learning by providing individualized access to information, immediacy in reflection, expedient and collaborative interaction, and convenience.

Lastly, participants within this study consistently referenced the full capacity of technology-based formative assessment applications to impact instruction, and thus student learning, has yet to be realized. Wiliam (2011) stated infusing technology alone would not yield the intended results of increased student achievement; however, the effective use of technology to enhance formative assessment and positively impact student achievement and learning can be used. Within this research, professional development with regard to technology tools and applications varies by site and teacher, and is highly dependent on teacher choice. Specifically, the research shows teacher participants received formal professional development training in applications such as 
IXL, ALEKS, and Schoology as well as other technology-based formative assessment tools. Although these trainings existed, the results of professional development did not yield a consistent implementation of strategies across learning environments. A successful plan for professional development, according to Wiliam and Black (2009), and supported by these findings would develop skills in eliciting evidence of learning to provide feedback, adjusting future lessons and learning outcomes, and allowing students to use feedback to improve performance.

As a result, this research illustrates teacher participants within this research do not possess the same skills and abilities to adjust and adapt future learning experiences in a consistent way. Data collected from technology-based applications also varies by site. This also indicates collaboration and reflection opportunities are not impacted by technology-based formative assessment with regard to specific learning standards, as there are no common data points to compare. As was observed in a teacher collaboration session, the extent to which technology-based tools was discussed was relative to how it would be embedded into the instructional unit. The embedding of technology in that circumstance was driven by ease of instruction, not increasing the amount of data related to the learning standard. As a result, the results of professional development relative to data collection and analysis in order to impact future learning experiences was inconsistent across participants, and limited to the extent of which teachers were willing to infuse within instructional practice. The results of this research indicate the infusion of the concept of technology-based formative assessment applications without a specific design for how they will be utilized, what data would be reviewed to reflect upon effectiveness, and what types of results would be analyzed to determine adjustments to 
instruction can result. Concurrently, teachers provided autonomy to choose which technology-based formative assessment applications were utilized, without setting minimum implementation standards, resulted in a variety of responses by teachers with regard to the effectiveness of technology-based formative assessment applications.

\section{Recommendations}

This study focused on highlighting the importance of embedding opportunities for technology-based formative assessement feedback within the teaching and learning cycle. The research revealed the timeliness and quality of feedback, as enhance by technology, impacted student learning in a positive capacity, and this inquiry supported that assertion. Following is the executive summary that will be presented to the district leadership team, instructional specialists, building leaders and mathematics teachers to enhance their understanding of how to technology can provide augmented or modified learning opportunities, as well as assist in developing a consistent district-wide plan for professional development that supports collaboration utilizing common data sets. In addition, the following executive summary highlights the importance technology in providing personalized, individualized, and immediate feedback to teachers and students on learning standards.

Recommendations for future studies of this nature are as follows. First, develop the study as a mixed-methods review of information to include quantitative data in the form of surveying teachers and analysis of student achievement data. The second recommendation for future study is to select multiple districts or sites to compare data on the results of different technology-based formative assessment tools. Finding schools in this area that do not utilize technology would be nearly impossible, eliminating the option 
of doing a comparison of formative assessment without technology. Finally, exploring the role of professional collaboration and professional development within the scope of how teacher training with technology-based tools impact student learning. The results of this research showed an emerging theme related to professional development and training, even without it being a specific research question.

Presented to district leaders will be the following executive summary. Made within the scope of the limitations of this inquiry are these recommendations. Moreover, it is the hope of the researcher this executive summary will be the basis of continued discussion on technology-based tools impact on student learning. 


\section{TECHNOLOGY-BASED FORMATIVE ASSESSMENT}

\section{The impact on teaching and student leaming in middle school mathematics}

As the acsessibility of technology devices asd technology-based applications has increased, researchers have attempsed to make coencctions between practices within teaching and kanaing, and how technology can be used to enhasoe $\propto$ deplete their effectiveness. Whes investigating technology withia education, elenents such as funding, progran initiatives, and the introduction of specific techsology standards isdicate a unified belief within the edscational community that technology is a secessary couponent of the educational process. Research on the effects of utilizing fonmative assessmsent strategies to provide fecdback, and therefore, affect the overall leaning of students, has beea conducted in various forms. Research on how the use of effective formative assessment strategies increases student leaning axd research to support how technology imtegration cas impact studext learning has been completed. Evidesce exikts to sapoont bow the effective wese of feedback and formative assessmeat can impact student leaning followed by the consideration of bow sechalogy can be used to impact student leaming through its we iv formative assessment.

The purpose of thls research ls to determine whether technology-based formative assesament applications impact student learning as a function of moblle learning theorles effecting the frequency. quallty, and use of feedback

\section{Research Questions}

1. Do technology-based tools asd applicatious impact the way teachers gather, analyze, asd uxilize infocmation obcained from fonmative assessments to impact studeat learning?

2. How do techoology-based tools (IXI., ALEKS, Schoology, Google Applications, or other ideatified applications within the study) increase studeat leaming as a functionef the way seachers and administrasors gather, analyze, and apply the information obtained?

3. How do technology-based formative assessment resounga impact the frequency and quality of foedbock uxilized by teachers to enhasoce teaching and karning?

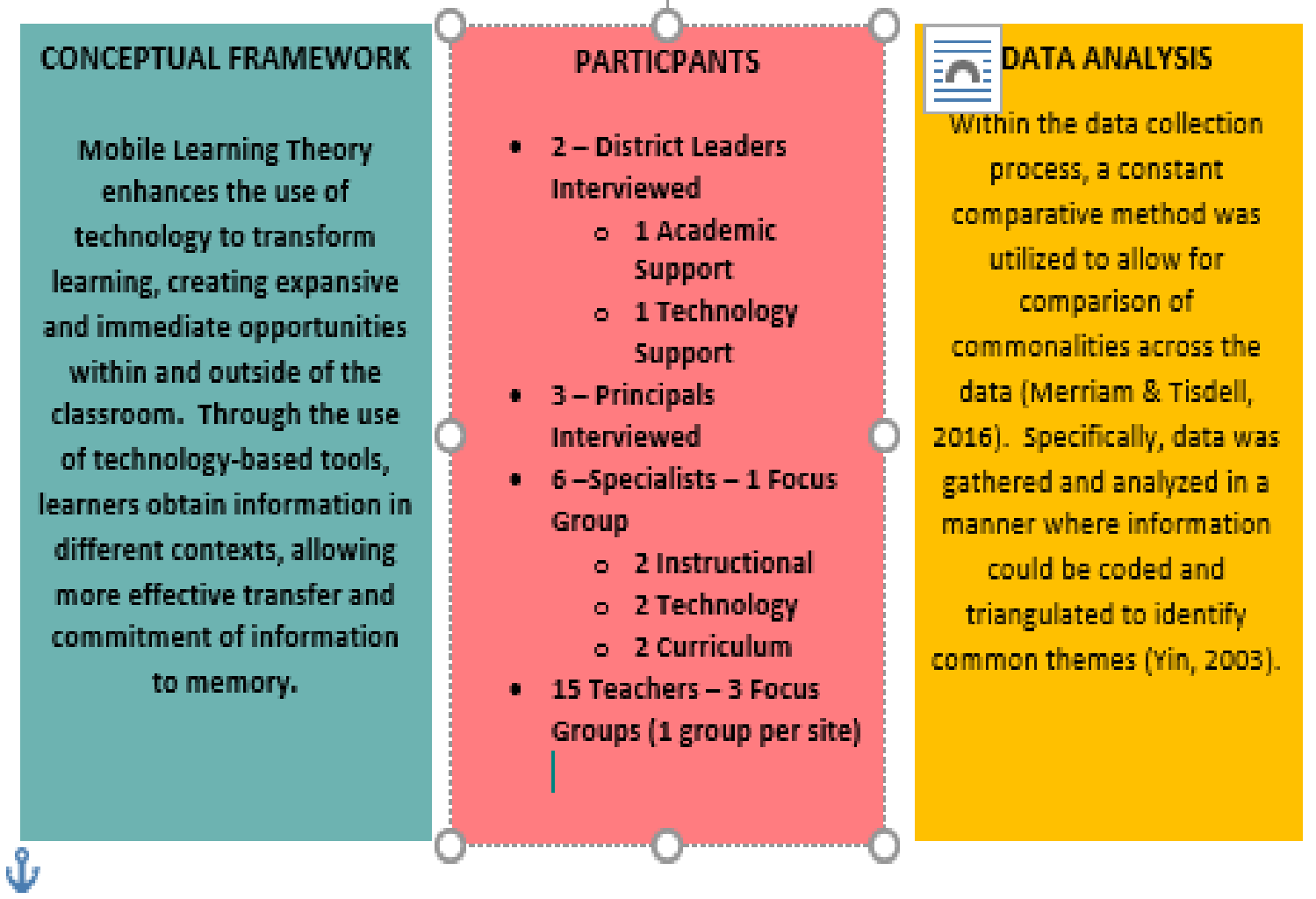




\section{Description of Organization Structure of the Participants}

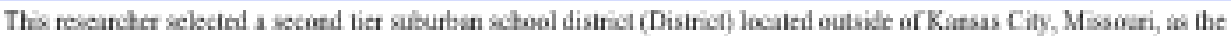

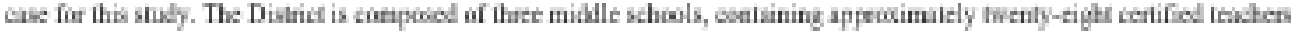

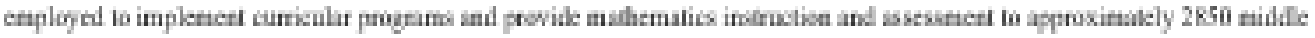

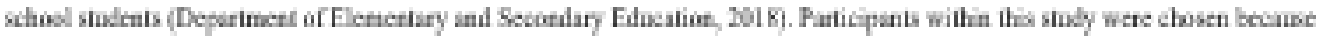

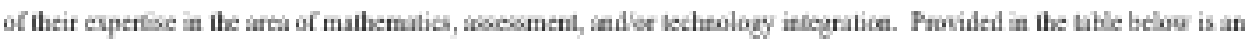

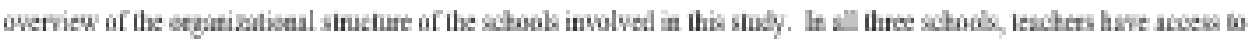

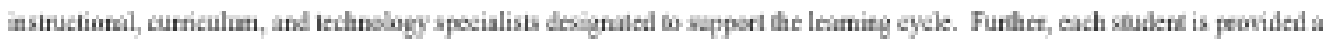

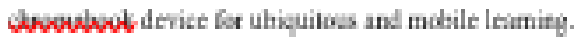

\begin{tabular}{|c|c|c|c|c|c|c|c|}
\hline \multirow[b]{2}{*}{ Bchal } & \multicolumn{2}{|c|}{ Bludent } & \multicolumn{3}{|c|}{ Tanshint } & \multicolumn{2}{|c|}{ 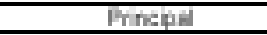 } \\
\hline & Eaminart: & FIM Lanth & $\begin{array}{c}\text { Tosil } \\
\text { Tantharim }\end{array}$ & be & 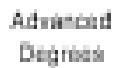 & Anghat & $\begin{array}{l}\text { Haghast } \\
\text { bepres }\end{array}$ \\
\hline A & 441 & $2 \pi$ & at & 14 & Benth & 14 & Desanat \\
\hline 日 & 194 & 25 & es & 12 & B. 35 & $\exists$ & A日D \\
\hline $\mathrm{c}$ & $1 \cdot 34$ & 114 & ab & 14 & B.t. & 7 & Decenta \\
\hline
\end{tabular}

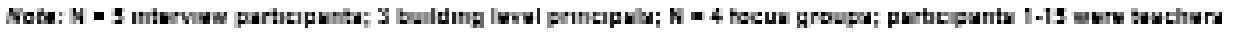

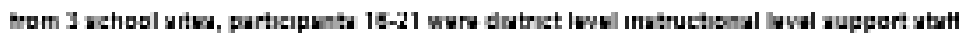

\section{Emerging Themes from Qualitative Data}

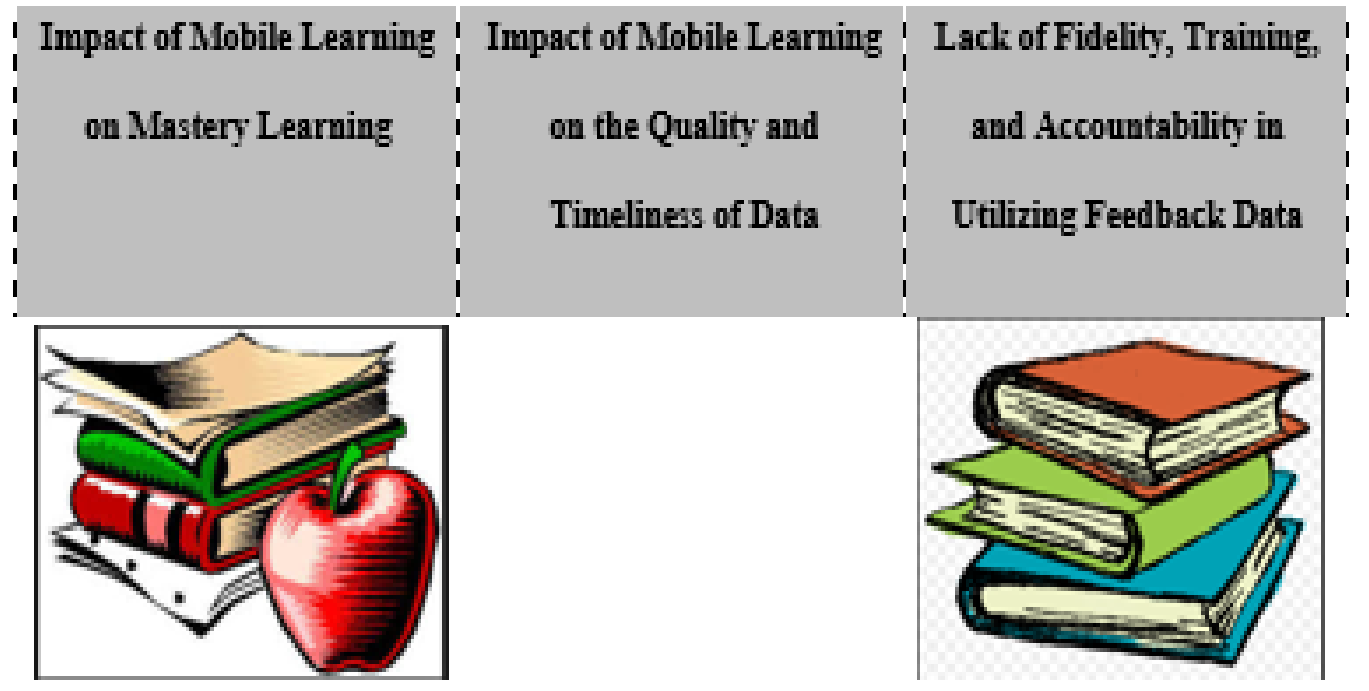




\title{
Findings
}

\begin{abstract}
Impact of Mobile Lesruing on Mastery Lesrnixg
The data collected from participans, observatioes, and documents suppooted a that iechnology infuxion withia the leanaing cycle is dependent on the purpose for infusioe. Participants provided evidence to suppoct an increase in outside leaming oppoctunities for studeats, such as tuonorials, videos, and instant feedback on failed kearning standurds. Futhermore, the programs primarily utilised by teachers offered expanded leaning oppontunities when mastery is achieved, as well as provided feedhack that encourages remodiation when aecessary. Therefore, augusentation and modification of learning occurred as a result of the expasded use of iochnology-based tools ousside of the classroom on specific leaning stasduds.

\section{Impact of Mobile Lesrning on the Quality and Timeliness of Data} Responses from all participars emphasized the use of IXL., ALFXS, and Schoology applicatioes provided an increased level of timely feedback to teachers and students. Data further supported the assertion that technologybused fonnative assessment tools provide accurate and imusediate feedback to stodents and teachers which allows for adjustusents to learning opportunities. The vision for technology infusioe was to provide more infocunation to teachers and studests, more froquently, and i a mooe efficient context í onder to maximize student learning. An increase in the frequency and innediacy of feedback had a positive effect on student learning through an increase in specific remediation tools asd strategies, although it does sot impact student leaning ualess students and teachers take advarage of the available resources for adjustmeat or remediation. The availability of immediate feedback, both withia and outside of the instructional time, provided iscreased leaming opportunities for students whe were motivased and chose to participate in supplemental learning activities.

\section{Lack of Fidelity, Training, and Accountability in Unlizing Feedback: Data}

Responses provided evidence to support teacher autooceny and choice when desermining which sechnology-based formative assessenent tools were utilized, as well as bow they were infused. Responses by participants identified over fifteen technology-based focmative assessment applicatioes being utilized withia the study. Specialist purticipants expanded on this topic stating teachers have acoess to an abundance of technology-based tools, as the district has focused on providing techoolagy-based fonnative assessment tooks to teacherc. As a result of implementation of techuology-based iools being volurvary and autooomous, the factors which impocted the selection of applications and bow leanaing was effecsed acmoss sijes were ibconsistent. La addition, the way in which consistently used applications were infused vaied across sises and teachers. All participunts indicated a deficiescy in teacher skill set relative to coemmon technology-bused tools. As such, the volurvary nature of utilizing technology-based formative applications, in coujunction the varying levels of teacher compesency, supporied assertions participants that tochuology-based applicatious have the ability to impact leanning more so than is being realized.
\end{abstract}

\section{Recommendations}

\section{Recommendation \#1}

Provide poofessional development relative to selected technology-hased applications.

Train teachers how to gather, analyze, and respond to data collected relative is the effectiveness of instruction

lnerease number of oppoctunities for instructional, technology, and curriculum specialists to ohserve and interact with teachers

\section{Recommendation \#2}

Utilize consisteat applicatioes acmos all sites in ocder to provide common data points for discussion, collaboration, and reflection.

Select specific leaning tangets where techoology can provide specific infocmation in the most effective way, and identify which leaming targets are to be eliminated from scepe of technology-hased applications bused on being more process related.

\section{Recommendation \#3}

Establish a criterion for the comunon (consistent across sites) data teachers are required to gather

Create a supervision plan to increase fidelity and accountability for urilizing sclected, noo-volumary applications and data points 


\section{SECTION FIVE}

CONTRIBUTION TO SCHOLARSHIP 
Technology-Based Formative Assessment: Technology’s Impact on Teaching and Learning in Middle School Mathematics

Brett A. Jacobs, Ed.D. 


\section{INTRODUCTION}

Research on the effects of utilizing formative assessment strategies to provide feedback, and therefore, affect the overall learning of students, has been conducted in various forms. Research on how the use of effective formative assessment strategies increases student learning (Black \& Wiliam, 2009; Hattie, 2012; Rutheford, 2013; Wiliam, 2011), and research to support how technology integration can impact student learning (Jude, Kajura, \& Birevu, 2014; Martin, 2015; Puentedura, 2013; Romrell, Kidder, \& Wood, 2014) has been completed. Nonetheless, a reasonable gap exists between technology-based formative assessment and its impact on student learning. Specific to instruction, researchers (Marzano, 2006; Vaughn, Cleveland-Innes \& Garrison, 2013) stated utilizing a community of collaboration and inquiry with the purpose of reflecting on practice, utilizing social learning opportunities, encouraging group learning, and enabling self-reflection can lead to gains in student achievement. Other researchers (Black \& Wiliam 1998, 2009; Wiliam, 2011) studied the origins and effects of utilizing formative assessment strategies to determine the overall impact on the instructional process and overall student learning. Explicitly, Wiliam (2011) offered evidence regarding how the effective use of feedback and formative assessment can impact student learning followed by the consideration of how technology can be used to impact student learning through its use in formative assessment.

\section{Statement of the Problem}

As technology, and technology applications have increased in accessibility and functionality, with the allocation of resources growing at a faster rate than research can support. The acceleration of technology infusion under the premise of increased learning 
potential often occurred before the development of a professional development plan to account for teacher training. As such, Wiliam (2011) stated infusing technology alone would not yield the intended results of increased student achievement. While past efforts of providing classrooms with technological tools such as computer workstations and Smart Boards increased the level of technology access, researchers (Jude et al., 2014; Wiliam, 2011) maintained initiatives in technology have failed to increase achievement as such efforts merely modified the method of providing equivalent instruction. According to Faber, Luyten, and Visscher (2017), research on the effectiveness of technology-based tools and their impact on formative assessment feedback is lacking. Furthermore, researchers asserted that infusing technology in classrooms where teachers are trained to enhance feedback and modify instruction based on information gained should be further studied (Vaughn et al., 2013; Wiliam, 2011).

This problem of practice will explore the concept of examining technology as a tool to increase speed, accuracy, and quality of feedback. Additionally, this study will further examine the impact of technology-based formative assessment on teaching practices that ultimately influence student learning. The impact of technology on the effectiveness of proven educational practices is an area that is in need of further exploration. Specifically, as schools make strides in increasing access to technology and technological applications, the impact of technology-based formative assessment to modify instruction and provide alternate learning opportunities for students will provide useful information as to the significance of such initiatives. 
dissertation in practice, B. Jacobs

\section{Purpose of the Study}

Researchers defined formative assessment as the intentional gathering of information to inform and improve teaching within the process of learning (Black \& Wiliam, 1998, 2009; Cowie \& Bell, 1999; Wiliam, 2011). This researcher will analyze formative assessment grounded in technology-based tools and the impact on student learning within $7^{\text {th }}$ and $8^{\text {th }}$-grade mathematics. The researcher will examine how the frequency of feedback within instruction impacts student performance through assessments designed to measure student success (Romrell et. al., 2014; Wiliam, 2011). Further, this researcher will explore the technology-based applications to identify which significant factors impact student results accounting for frequency, informal collaborative learning experiences, and instructional efficiency (Looi et. al., 2009). Moreover, the researcher will study the impact of technology-based formative assessment applications on preparation and collaboration, and whether applications affect student performance related to designed assessments (Hwang \& Chang, 2011; Nicol \& Macfarlane-Dick, 2006; Reeves, Gunter \& Lacey, 2017).

\section{Research Questions}

The following research questions guided the present study:

1. How do technology-based tools and applications impact the way teachers gather, analyze, and utilize information obtained from formative assessments to impact student learning?

2. How does the professional development and training of teachers and administrators specific to technology-based tools and applications (IXL, ALEKS, 
dissertation in practice, B. Jacobs

Schoology, or other applications) impact the way teachers adapt instructional learning opportunities?

3. How do technology-based formative assessment resources impact the frequency and quality of feedback utilized by teachers to enhance teaching and learning?

\section{Conceptual/Theoretical Framework}

This researcher will examine the theoretical framework of learning theory to determine the impact of technology on student learning. Behaviorism, Cognitivism, Connectivism, and Mobile Learning (MLT) theories will be utilized to assess whether learning occurs independent of, or in conjunction with, technology-based and social constructivist methodology. Infusing technology within learning experiences will be examined utilizing the SAMR model, which depicts four levels of technology integration (Jude et. al., 2014; Puentedura, 2013; Romrell et. al., 2014). As previously stated, the purpose of this research is to determine whether technology makes an impact on student learning through the stakeholders perceptions. The SAMR model provides a template for evaluating the effectiveness of technology incorporation and allows for analysis of the impact of technology on learning through the frameworks.

\section{Behaviorism}

When considering the behaviorist approach, the intended function of technology integration results from changing the responses of students by introducing new stimuli. (Ally, 2004; Boghossian, 2006; MacCallum \& Parsons, 2016; Ormrod, 2012). As variables in this case, introduced are technological applications, since learning occurs as a function of reinforced responses based on observable behaviors. Behaviorism supports learning as a sequence of events independent of internal processing or reflection (Ally, 
2004). As such, behaviorism-learning theory met the substitution level criterion, where technology-based learning activities existed as a function of replacing traditional learning experiences (Puentedura, 2013; Romrell et. al., 2014).

\section{Cognitivism}

Explored as an alternative to the behaviorist learning theory was cognitivism, as researchers indicated it better explains how learners process information as a part of teaching and learning (Berliner, 2006; Bransford et. al., 2000; Yilmaz, 2011). Tenets of cognitive learning allow for comparative analysis of prior knowledge and construction of new information. Learners are required to use logic to make meaning of the learning experience, and thus commit new information to memory as a rote response (Berliner, 2006). Cognitivism provides a deeper venue for exploring technology than behaviorism by incorporating variables such as feedback and social interaction. However, cognitivism fails to explain learning experiences beyond the non-technology enriched activity.

\section{Connectivism}

Connectivism learning theory evolved in response to the need to explain how technology changes the process of learning (Barry, 2013; Kizito, 2016; Ozan \& Kesim, 2011; Siemens, 2014). One primary principle of connectivism is that technology redefines knowledge to be an interface with information (Bell, 2011; Downes, 2008; Siemens, 2014). Traditional theorists define knowledge as information processed, transferred, and committed to memory, thus making it a tangible set of acquirable facts (Boghossian, 2006; Conradie, 2014; MacCallum \& Parsons, 2016). Connectivism defines knowledge as the ability to access information through connections made possible using technology (Anderson \& Dron, 2011; Kop \& Hill, 2008). Technology 
within connectivism provides a pathway to knowledge and serves as a tool for increased access to information (Kizito, 2016; Siemens, 2014). As such, connectivism-learning theory places emphasis on creating pathways to information that can be continually accessed and updated in real time (Siemens, 2014). Although connectivism provides a venue to connect technology and learning, a gap still exists between its principles and those of formative assessment feedback.

\section{Mobile Learning Theory}

Mobile learning theory (MLT) utilizes technology to transform learning, creating instant and expansive opportunities within and outside of the classroom for learning to occur (Romrell et. al., 2014; Sharples, Taylor, \& Vavoula, 2007). Technological tools and applications can be utilized to substitute, augment, modify, or redefine traditional learning activities in a way that maximizes the experiences for effective instructional design (Puentedura, 2013; Romrell et. al., 2014). Researchers also asserted through use of technology-based tools that learners could obtain information in different contexts, thus allowing for a more effective transfer and commitment of information to memory (Hwang \& Chang, 2011; Pachler et. al. 2010; Puentedura, 2013; Romrell et. al., 2014). MLT emphasizes incorporating feedback and formative assessment as a part of the instructional process (Burns, Klingbeil, \& Ysseldyke, 2010; Dyson \& Frawley, 2016;

Lee, Feldman, \& Beatty, 2012). This researcher determined MLT as the conceptual framework that allows for examining the augmentation or redefinition of learning activities, which researchers (Puentedura, 2013; Romrell et. al., 2014) described as expanding learning experiences beyond what is capable without technology. 
dissertation in practice, B. Jacobs

\section{Design of the Study}

Merriam and Tisdell (2016) identified an essential component when designing qualitative research as one to determine how to design the study because of the theoretical framework in connection to the purpose of the study. Researchers within a basic qualitative study gather information related to how people interpret experiences, construct their worlds, and the meaning in which they attribute to those experiences (Creswell, 2014; Merriam \& Tisdell, 2016). Within this basic qualitative study, the researcher will utilize a case study design as outlined by Creswell (2014) and Merriam and Tisdell (2016). Researchers (Baxter \& Jack, 2008; Creswell, 2014; Merriam \& Tisdell, 2016) described a case as an analysis of information gathered from multiple sites and a variety of participants visited, observed, or interviewed. Further, Merriam and Tisdell (2016) outlined the function of a case study is to analyze a phenomenon within a bounded system, such as a person, program or event. In this case study, the case is identified as how technology-based formative assessment feedback impacts student learning in the three selected sites within the District. In this study, the researcher will identify teachers, principals, and district personnel with explicit information pertinent to the impact of technology-based formative assessment within the sites and District. Analyzing data collected through interviews, focus groups, observations, and document analysis provides evidence when identifying and interpreting the meaning within recurring themes (Krueger \& Casey, 2014; Merriam \& Tisdell, 2016). The use of a bounded case study will allow the researcher to solicit data to inform potential conclusions. 
dissertation in practice, B. Jacobs

According to Baxter and Jack (2008), once a case study and its boundaries are established, researchers should next consider the type of case study to conduct, selecting a case design guided by the purpose of the study. Yin (2003) outlined designs of case studies to be: 1) explanatory - creating causal linkage between program implementation and program effects, 2) exploratory - where interventions have no clear set of outcomes, or 3) descriptive - to describe a phenomenon and the real-life context in which it occurred. This study will use an explanatory case study design to determine whether the phenomenon, integrating technology within formative assessment, creates an effect of increased student learning. As such, the goal of the researcher is to rely on the views of the participants utilizing broad, generalized, questions allowing for discussion or interaction to construct meaning (Creswell, 2014). Crotty (1998) asserted meaning is constructed, not discovered, within the interpretation of an experience or social interaction. Therefore, the researcher will operate within the paradigm of social constructivism, the belief that, "individuals seek understanding of the world in which they live and work" (Creswell, 2014, p. 8).

Social constructivism, according to researchers (Amineh \& Asl, 2015; Creswell, 2014), is a theory of knowledge where knowledge is constructed jointly and in coordination with others to create meaning of a constructed reality. Within social constructivism, the essential components of the paradigm focus on the production and construction of knowledge as a group, where learners construct knowledge and meaning of experiences with other members of the learning community (Doubleday et. al., 2015; Vygotsky, 1978). Schunk (2012) asserted teaching from a social constructivist perspective emphasizes strategies that promote learning with others, such as reciprocal 
teaching, problems-based instruction, and peer collaboration. Teachers play the role of the facilitator and allow for the construction of understanding by individual learners (Amineh \& Asl, 2015).

Creating an environment of interaction, such as increasing the role of technology, as a function that supports learning inherently increases the opportunity for teachers and students to construct meaningful learning opportunities beyond traditional core skills (Kivunja, 2014). Social constructivism provides a paradigm of learning for teachers, as developing strategies for meaningful teaching and learning with technology is dependent on the collective experiences of the group. In this case, teachers play the role of the learner, reflecting upon benefits and detriments of technology-based formative assessment with each experience, therefore constructing their own inferences and learning connected to its impact on student learning. In addition, this study will create an opportunity for participants to discuss collectively their experiences within teaching and learning related to technology-based formative assessment and its impact on student learning. The constructed knowledge of participants within this qualitative study will allow the researcher to triangulate information, present emerging themes and formulate conclusions relevant to the research questions (Merriam \& Tisdell, 2016; Yin, 2003).

\section{Data Collection}

To utilize the program for this research, the Superintendent of Schools, by way of the DIT consented to the scope of this study by signing the gatekeeper consent form (see Appendix A). The DIT is composed of the district Superintendent and other leaders within the district whose responsibilities include instruction, professional development, facilities, technology, and human resources. Then, the proposal was be submitted to the 
dissertation in practice, B. Jacobs

University of Missouri's Institutional Review Board (IRB). Once IRB approval was received (see Appendix B), the researcher began data collection for the proposed study. Consent was needed from teachers, instructional coaches and technology integration staff (See Appendix C), as well as building and district administrators (see Appendix D) utilizing an informed letter of consent. Utilizing coding to identify participants (see Appendix $\mathrm{H}$ ), the researcher anonymously presented information and data to support emerging concepts and themes.

During the process of collecting data for this case study, several sources were utilized. These included interviews with building and district administrators, focus groups with mathematics teachers and district instructional support staff, analysis of district and building documents, and observation daily activities. In addition, this researcher conducted an in-depth examination of relevant literature related to how learning is impacted by feedback (Andersson \& Palm, 2017; Beatty \& Gerace, 2009; Black \& Wiliam, 2009; Wiliam, 2011), cooperative and collaborative learning experiences (Magaña \& Marzano, 2014; Marzano, 2006), and technology-enhanced instruction enhanced within mobile learning (Crompton, 2013; Hwang \& Chang, 2011; Puentedura, 2013; Reeves et. al., 2017; Romrell et. al., 2014; Xie, 2016).

Within qualitative research, researchers (Creswell, 2014; Merriam \& Tisdell, 2016; Yin, 2003) asserted it is necessary to take steps before collecting data that will increase validity, reliability and transferability of findings. According to Creswell (2014), "Validity and reliability of scores on instruments lead to meaningful interpretations of data" (p. 155). To accomplish this goal, this researcher chose to conduct a pilot, or field test: a method described by researchers (Creswell, 2014; Dikko, 
2016, Merriam \& Tisdell, 2016) as a mini version of the research study used to increase the validity of the instruments used within the study. Researchers described face validity as a subjective analysis to determine whether the measurement makes sense, and is measuring the intended target (Nevo, 1985; Wheeler, Goolkasian, Baird, \& Darden, 1999). Further, Dikko (2016) describes content validity as ensuring "the instrument of measurement has tapped the concept it sets out to measure by including an adequate representation of items that operationalize the concept" (p. 521). The researcher utilized results from pilot testing to improve the data collection protocols, thus increasing face and content validity to improve accuracy when interpreting findings. An additional objective of pilot testing is to increase the internal validity, or credibility, of findings, thus creating a study that better captures reality and strengthens the argument in support of the findings (Merriam \& Tisdell, 2016).

\section{Focus Group Protocols}

Focus groups are a method to collect qualitative data through the opinions, statements, mannerisms, and group dynamics of participant groups (Creswell, 2014; Gill, Stewart. Treasure, \& Chadwick, 2008; Krueger \& Casey, 2014). The researcher conducted on-site, semi-structured, focus groups composed of mathematics teachers $(\mathrm{n}=$ 15-20) to obtain information regarding the implementation and effectiveness of technology-based formative assessment feedback on student learning (see Appendix B). Additionally, a focus group was conducted comprised of the district-level curriculum, technology, and instructional specialists $(\mathrm{n}=5-7)$. The focus group protocols (see Appendix B) were developed as a series of questions guided by the research questions, and designed to provide information regarding the impact technology-based formative 
assessment feedback has on student learning and achievement in $7^{\text {th }}$ and $8^{\text {th }}$-grade mathematics. Once recordings were transcribed, members of each focus group were provided a transcript to verify the context and content of their statements. Researchers (Creswell, 2014; Lincoln \& Guba, 1985; Seidman, 2013) define member checking as a practice utilized by researchers to validate the statements and opinions of members within a group or interview to increase trustworthiness and credibility of the report. Focus groups occurred on site or via video conference and lasted approximately sixty to ninety minutes.

Questions within the focus group protocols (see Appendix B) were designed to elicit information specific to the knowledge, abilities, application, and access to technology of the participants as related to formative assessment feedback within the teaching and learning cycle. The questions within this protocol focused on obtaining information relative to teachers knowledge of formative assessment feedback strategies (Black \& Wiliam 1998; Cowie \& Bell, 1999; Wiliam, 2011), skill set regarding embedding formative assessment and technology within their lesson design (Crompton, 2013; Hwang \& Chang, 2011; Puentedura, 2013; Reeves et. al., 2017; Romrell et. al., 2014; Xie, 2016) and perception of professional development in regard to both formative assessment and technology integration. This process allowed the researcher to develop focus group questions specifically designed to elicit responses relevant to the experiences and expertise of the participants as it related to the impact of technology tools and applications on formative assessment feedback.

After focus group questions were developed, a pilot study of the focus group protocol questions was conducted with a selection of $7^{\text {th }}$ and $8^{\text {th }}$-grade mathematics 
teachers $(n=2-4)$, and instructional specialists $(n=1-2)$ independent of those chosen to participate in the study. Researchers (Creswell, 2014; Fink, 2015; Krueger \& Casey, 2014; Siedman, 2013) stated pilot testing allows the researcher to adjust instrumentation based on the feedback obtained from participants of the pilot test, thus improving the questions and format of the data gathering process. Using the results and feedback from pilot testing of focus group protocols allowed the researcher to ensure questions address the desired content area and make adjustments to questions as needed before conducting the study, thus increasing the studies content validity (Creswell, 2014; Dikko, 2016; Fink, 2015; Merriam \& Tisdell, 2016). Ultimately, pilot testing increased content validity within focus group protocols, as the researcher was able to identify whether the questions elicit responses relevant to the topic of student learning related to formative assessment feedback and MLT. In addition, researchers outlined the utilization of pilot testing to adjust the wording and format of questions as a method of increasing the face validity of the protocols (Nevo, 1985; Wheeler, Goolkasian, Baird, \& Darden, 1999). As such, the researcher worked with participants to determine whether the questions were constructed appropriately, to identify areas of ambiguity, and to ensure questions were clearly understood (Creswell, 2014; Krueger \& Casey, 2014). Thus, adjustments to the protocols based on the feedback from the pilot study increased the content and face validity of the focus group protocols.

\section{Interview Protocol}

Researchers (Seidman, 2013; Schutz, 1967; Van Manen, 2015) stated the interview process should elicit the subjective experiences of participants resulting in a retrospective analysis of the meaning of lived experiences, thus revealing a particular 
point of view. The purpose of in-depth interviewing is to explore the experiences of people as a function of the meaning they associate with those lived experiences (Seidman, 2013). Seidman further highlighted the recognition and affirmation of the role of the interviewer as a skillful and intentional adaptation of the instrumentation. When interviewing building administrators, the researcher utilized a phenomenological approach to interviewing with an emphasis on technology-based formative assessment. As such, the researcher designed a series of open-ended, clarifying, probing and followup questions to "encourage participants to engage in that 'act of attention' that then allows them to consider the meaning of lived experience" (Seidman, 2013, p. 19). The researcher developed an interview protocol form (See Appendix B) to organize data collected in an accessible format for analysis and triangulation. The interview protocol contained eleven questions developed by the researcher, guided by the research questions, and conducted in a semi-structured format to identify the impact of technology-based formative assessment feedback on student learning. Interviews were scheduled to be approximately one hour in length.

Questions within the interview protocol (Appendix B) were designed to identify how formative assessment feedback and technology integration, singularly or in conjunction with one another, are incorporated within the district plan for teaching and learning. Further, questions within the designed interview protocol were intended to obtain the perceptions of participants related to the literature specific to formative assessment feedback and MLT to triangulate information gathered from other data collection methods (Seidman, 2013). Ultimately, the questions were designed to determine the specific perception of building and district level administrators regarding 
formative assessment feedback and technology integration as it relates to the district Comprehensive School Improvement Plan (CSIP). As stated by researchers (Marzano, 2014; Rutheford, 2013; Wiliam, 2011), meaningful feedback provided as a function of peer collaboration, self-reflection, or instructor review has a positive impact on student learning. A successful plan for professional development, according to Wiliam (2011), develops skills in eliciting evidence of learning to provide feedback, adjusting future lessons and learning outcomes, and allowing students to use feedback to improve performance.

After interview questions were developed, a pilot study of the interview protocol questions was conducted with a selection of building and district level administrators $(n=1-2)$ independent of those chosen to participate in the study. As previously stated, pilot testing allows researchers to increase the content validity of the instrumentation (Creswell, 2014; Seidman, 2013). The purpose of the pilot test was to increase content validity by analyzing whether questions elicit responses relevant to the desired content of the study (Fink, 2015; Siedman, 2013). A secondary purpose of the pilot test was to increase face validity, which was accomplished through discussion with participants as to whether the questions are clearly understood and identify areas of ambiguity within the questions or process (Creswell, 2014; Fink, 2015; Krueger \& Casey, 2014). In this case, the researcher used feedback from the participants to adjust and reformat questions within the interview protocol to insure questions were clear, as well as elicit responses relevant to the content of the study. The pilot test of interview protocols increased the content and face validity of the instrumentation and the validity of potential findings. 


\section{Document Protocol}

Researchers described documents as an array of evidence in written, visual, digital and physical form to understand realities, communicate information, and produce insight to a conclusion of research (Flick, 2009; Merriam \& Tisdell, 2016). Public records within qualitative research are official and ongoing documents within an institution and manifest in a variety of forms (Creswell, 2014; Merriam \& Tisdell, 2016). The researcher created a protocol for document analysis (see Appendix C) to catalog information related to the topic within the District. Documents will be chosen specifically related to gathering information related to how technology is acquired, maintained, and utilized for instruction. Furthermore, document analysis allowed for an examination of curriculum guides, assessments, teacher development related to technology, and results of data collected by the district regarding technology tools and applications. By utilizing documents, the researcher decreased the chances of a biased study that could affect the validity of results (Creswell, 2014). Further, triangulating data with results from focus groups, interviews, and observations allowed the researcher to examine the impact of technology-based formative assessment tools on specific learner objectives (Yin, 2003).

\section{Observation Protocol}

Observations within a qualitative research study provide recorded data within a social setting, including but not limited to interactions, conversations, artifacts, events and behaviors (Marshall \& Rossman, 2014; Merriam \& Tisdell, 2015; Patton, 2014). In this case, observations allowed the researcher to participate in what Patton (2014) referred to as formal and planned opportunities, where an observer has an opportunity to 
see a phenomenon unfold in a semi-natural fashion. Patton described the benefit of personal contact as being able to observe and understand the "context within which people interact - for understanding context is essential to a holistic perspective" ( $p$. 332). Where interviews and documentation provide an interpretive analysis of a particular phenomenon, systematic observation provides a firsthand account of events, allowing triangulation of collected data incorporating senses other than sight that can validate results (Merriam \& Tisdell, 2015; Yin, 2003). The researcher developed an observation protocol (see Appendix D), and observed a meeting where teachers discussed how technology-based formative assessment feedback is incorporated within the learning cycle. The researcher participated in what researchers (Gold, 1958; Schensul \& LeCompte, 2012) described as a participant-as-observer role, building relationships with the group and participating in the activities while gathering data. The second observation setting was conducted on-site through a meeting conducted by the administrative leadership team. The researcher participated in an observer as participant role where gathering data is the primary focus of observation and building a relationship with participants will allow information to be transferred (Gold, 1958, Merriam \& Tisdell, 2015). The final observation was conducted at the district-level through participation in a meeting with district leaders of curriculum, testing, data analysis, and technology. The researcher continued in an observer as participant role to determine if the district CSIP addresses the implementation of technology-based formative assessment. The data collected in these observations allowed the researcher to form conclusions regarding outcomes of technology-based formative assessment. 
dissertation in practice, B. Jacobs

\section{Data Analysis}

According to researchers (Creswell, 2014; Flick, Scott, \& Metzler, 2014), qualitative analysis allows the researcher to purposefully select sites or individuals to gather and analyze data that best fit the research questions designed to obtain useful information. Within the data collection process, a constant comparative method was utilized to allow for comparison of commonalities across the data (Merriam \& Tisdell, 2016). Specifically, data was gathered and analyzed in a manner where information could be coded and triangulated to identify common themes (Yin, 2003). Seidman (2013) contended creating a systematic process for filing transcripts, tracking participants, accurately labeling recordings, and securing information are necessary steps in managing information. The researcher examined data utilizing codes (see Appendix H). Transcripts and field notes were read to identify and code emerging themes in order to reduce the risk of a biased analysis (Seidman, 2013). To code the transcripts, each line was numbered consecutively to provide structure to the coding process. The researcher read all transcripts to associate responses from participants about the research questions. The researcher used a coded color system to match interview and focus group responses to corresponding research questions. Once responses are sorted, an analysis of responses from all participants was conducted to identify emerging themes. According to Tesch (2013), a researcher sorts through collected data to define categories and their properties, at which time a comparison between categories can be analyzed. Collecting and analyzing information while utilizing a variety of qualitative methods and data sources to identify the emerging theme is referred to as triangulation (Creswell, 2014; Fowler, 2004; Merriam \& Tisdell, 2016; Yin, 2003). Furthermore, Merriam and Tisdell (2016) 
suggested interviews and focus groups be recorded and transcribed by the researcher.

The researcher provided a transcript to participants to complete the member checking process (Creswell, 2014; Seidman, 2013). Member-checking was completed to account for misunderstood or potentially biased information throughout the process (Creswell, 2014). Member-checking also allowed the participants to provide additional information that may not have been discussed within the focus group or interview process. (Creswell, 2014; Lincoln \& Guba, 1985; Seidman, 2013). In addition to recorded data, the researcher took field notes to document information not reflected on recorded focus groups or interviews, a method Krueger and Casey (2014) described to enhance information gathered.

\section{Limitations and Assumptions}

Limitations exist within all studies and considered by researchers before formulating conclusions or making recommendations (Creswell, 2014). Identifying the potential of internal and external threats aided the researcher when designing the study and increased the validity of findings (Creswell, 2014). It is imperative within qualitative research to check for accuracy, as well as use consistent methods when gathering and analyzing data (Creswell, 2014). Furthermore, increasing reliability within the findings by using valid and consistent methods increased the authenticity, credibility, and trustworthiness of conjectures (Creswell, 2014; Lincoln, Lynham, \& Guba, 2011).

Further, the researcher considered the steps the district has taken to increase access to devices, applications, and software, including measures to create an infrastructure conducive to technology infusion. Application of the information gathered within this study may prove useful to other schools and school districts, but will have to 
consider the variables within each setting individually (Creswell, 2014). Personal bias was another limitation of this study, as the researcher worked within the setting during the time of data collection (Creswell, 2014), creating a working knowledge that could inadvertently insert information into the findings not gathered by the designated instruments; i.e. focus groups, interviews, document analysis, and observation data. To account for potential limitations, bias, or validity of findings, this researcher utilized member checking and triangulation (Creswell, 2014, Yin, 2003).

\section{Discussion of Findings}

Creswell (2014) outlined the process qualitative data analysis to include data collection and write-up of findings. In conjunction, within the analysis of the data, the researcher was required to identify key aspects of the data, while discarding other information gathered within the study, a process defined as winnowing (Guest, MacQueen, \& Namey, 2012). This analysis come in the form of emerging themes from interviews of district and site leaders, focus groups with instructional specialist and teacher focus groups, observation of meetings, and analysis of documents from the district. Three emerging reoccurring themes evolved as the data was triangulated: The impact of mobile learning on mastery learning; The Impact Mobile Learning on the Quality and Timeliness of Data; Lack of fidelity, training and accountability

\section{Impact of mobile learning on mastery learning}

Mobile learning theory utilizes technology to transform learning, creating instant and expansive opportunities within and outside of the classroom for learning to occur (Romrell et. al., 2014, Sharples, Taylor, \& Vavoula, 2007). Technological tools and applications can be utilized to substitute, augment, modify, or redefine traditional 
learning activities in a way that maximizes the experiences for effective instructional design (Puentedura, 2013; Romrell et. al., 2014). The data collected from participants, observations, and documents supported a conclusion which is indicative of the level of technology infusion within the learning cycle is dependent on the purpose for infusion and the effect technology had on the identified learning standard. Participants provided evidence to support an increase in outside learning opportunities for students, such as tutorials, videos, and instant feedback on failed learning standards. Furthermore, principal participants observed the programs primarily utilized by teachers offered expanded learning opportunities when mastery is achieved, as well as provided feedback that encourages remediation when necessary. Researchers asserted through use of technology-based tools that learners could obtain information in different contexts, thus allowing for a more effective transfer and commitment of information to memory (Hwang \& Chang, 2011; Pachler et. al. 2010; Puentedura, 2013; Romrell et. al., 2014). As such, mobile learning theory researchers, (Burns, Klingbeil, \& Ysseldyke, 2010; Dyson \& Frawley, 2016; Lee, Feldman, \& Beatty, 2012), in addition to collected data, promoted the incorporation of technology-based feedback and formative assessment as a part of the instructional process.

According to Dyson, Litchfield, Raban, and Tyler (2009), the interactive systems available within mobile learning de-emphasize traditional teacher-centered models and encourage an active learning processes. This is evident in how teacher participants utilized technology-based applications to provide learning experiences to students outside of the school day. Teacher participants identified remediation and self-monitored learning of specific learning standards outside of instructional time as beneficial to the 
implemented technology platforms. Affective utilization of mobile devices and applications must consider effective instructional design when using applications and tools to augment and modify learning experiences. This was evident throughout the data collected, and in the work of researchers (Hwang \& Chang, 2011; Kihoza, Zlotnikova, Bada, \& Kalegele, 2016; Pachler, Daly, Mor, \& Mellar, 2010) who concluded the utilization of technology augments the teaching and learning process by increasing the functionality of formative assessment feedback. Teacher participants within this study, as well as district instructional specialists, asserted technology-based formative assessment tools allowed for learning experiences to occur within, and outside of the instructional space that would not occur otherwise without technology.

The SAMR model provides a framework for classifying activities when utilizing technology, allowing instructors to identify a purpose for mobile technology integration (Jude et. al., 2014; Romrell et. al., 2014). Researchers of the SAMR model (Jude et. al., 2014; Puentedura, 2013; Romrell et. al., 2014) asserted the use of technology can enhance and transform learning experiences and directly impact student learning and achievement when implemented beyond the substitution level. Analyzing the results of mobile learning activities to increase the quality and regularity of formative feedback to students will maximize the effect of MLT activities on student learning (Cochrane, 2012; Wong, 2016). Furthermore, Dyson and Frawley (2016) stated the implementation of MLT dramatically improves formative assessment feedback and marks.

When technology-based formative assessment tools are utilized as a substitute for traditional methods, participants communicated the reasoning behind the selection is often because they identified substitution as the best method of addressing the learning 
standard. Statements by teacher participants consistently referenced the inability of technology-based tools to provide information within certain processes students use to address learning standards. As noted by Teacher F, "math is a very process-based subject...you want to see their process and that requires seeing their work, and math is hard to type." Therefore, augmentation and modification of learning occurred as a result of the expanded use of technology-based tools outside of the classroom on specific learning standards. According to Duijn et. al. (2017), feedback should come from a credible source, address strengths and improvement areas, provide specific instructions for improvement, be provided immediately and should occur regularly. Data from participants indicated technology-based applications, such as IXL and ALEKS, accomplished each of these qualifying standards when infused with fidelity. Furthermore, all participants indicated

In addition to the characteristics previously communicated, Xie (2016) conveyed the utilization of mobile learning strategies to provide feedback and tips allows students to obtain feedback from multiple locations, contains individualized learning content specific to student response, and occurs in a nearly instantaneously fashion. Data collected indicated technology-based formative assessment tools utilized by mobile devices allowed learning to be personalized, transferrable, and differentiated based on student ability. Data collected from teacher participants indicated the traditional practice for students (worksheets) meant you had to give the same type of practice to all students and ask they complete it in the same timeframe. Further, the use of technology-based formative assessment applications allowed students to practice at the level of competency within and outside of the instructional space. Data from all participants further suggested 
technology-based tools encouraged students to work in the same learning standard until mastery. The triangulated data collected from all sources within this study also indicated the individualization of instantaneous feedback because of technology-based tools positively affected and expanded student learning. All participants, as well as district documents, supported the assertion that utilizing functions within the technology-based tools allowed for an increased opportunity for students to assess their own skills, correct mistakes and revisit specific learning standards.

\section{Impact of mobile learning on the quality and timeliness of data}

When connecting MLT and formative assessment, Hwang \& Chang (2011) explored formative assessment-based mobile learning as an approach for utilizing mobile devices to provide immediate feedback to students during learning activities. Further, Conejo, Garcia-Vinas, Gaston and Barros (2016) employed a web-based tool to incorporate Technology Enhanced Formative Assessment that provided elaborate and immediate feedback, and thus concluded students utilizing the designed tool outperformed those utilizing traditional formative assessment methods. While research on the effects of mobile learning on student achievement are found to be beneficial, Afshari et. al. (2009) asserted teacher ability within technology integration, quality of hardware and software resources, available time for collaboration within staff members, and lack of training and support for teachers within technology infusion are barriers to teacher implementation of mobile learning strategies. According to Ekanayake and Wishart (2014), the variables associated with teacher training and support have not been explored with fidelity within mobile-learning research. 
dissertation in practice, B. Jacobs

Responses from all participants emphasized the use of IXL, ALEKS, and Schoology applications provided an increased level of timely feedback to teachers and students. Data from principal participants further supported the assertion that technology-based formative assessment tools provide accurate and immediate feedback to students and teachers which allows for adjustments to learning opportunities.

Evidence obtained from district leaders outlined the vision for technology infusion was to provide more information to teachers and students, more frequently, and in a more efficient context in order to maximize student learning. All participants agreed an increase in the frequency and immediacy of feedback had a positive effect on student learning through an increase in specific remediation tools and strategies.

Conversely, data from specialist and teacher respondents indicated immediate feedback would not impact student learning unless students and teachers take advantage of the available resources for adjustment or remediation. Teacher participants indicated the availability of immediate feedback, both within and outside of the instructional time, provided increased learning opportunities for students who were motivated and chose to participate in supplemental learning activities. Leader, principal and specialist participants provided data to support a positive effect on student learning when teachers utilized information to adjust and adapt learning activities, including the addition of modified, re-teaching opportunities when data suggested a lack of proficiency. Crompton and Burke (2017) asserted the need to provide practitioners with a guide for utilizing and effectively implementing mobile learning, reducing the emphasis on student-centered initiatives and influencing student learning through instructional design. Although responses indicated a lack of adjustment by students and teachers could nullify the impact 
of feedback, participants overwhelmingly supported an assertion that technology-based tools provided immediate feedback on student progress within specific learning standards more frequently, and thus had a positive impact on student learning.

\section{Lack of fidelity, training, and accountability in utilizing feedback data}

When referencing technology-based formative assessment, utilizing technology augments the teaching and learning process by increasing the functionality of formative assessment feedback, therefore enhancing student learning through reflection, collaboration, real-time adjustment to learning activities (Hwang \& Chang, 2011; Kihoza, Zlotnikova, Bada, \& Kalegele, 2016; Pachler, Daly, Mor, \& Mellar, 2010). Researchers (Heitink, Van der Kleij, Veldkamp, Schildkamp, \& Kippers, 2016) asserted formative assessment should include "substantial, constructive and focused feedback" (p. 50). Rochelle, Penuel, and Abrahamson (2004) identified four constructs to unify technologybased instruction. Those constructs are: a) formative assessment, b) driving discussion by important conceptual contrasts, c) shifting to mastery-oriented motivational incentives, and d) harnessing diversity for generativity. Feldman and Capobianco (2008) expanded on the four constructs by further identifying the influence of curriculum integration and incorporating methods for constructing formative assessment items as components relevant to impacting teachers' practice.

While one objective communicated by all participants was to increase frequency and timeliness of formative assessment feedback, data from participants indicated a large number of technology-based tools, which were being utilized inconsistently across sites. Leader and specialist responses provided evidence to support teacher autonomy and choice when determining which technology-based formative assessment tools were 
utilized, as well as how they were infused. Therefore, responses by participants identified over fifteen technology-based formative assessment applications being utilized within the study. Specialist participants expanded on this topic stating teachers have access to an abundance of technology-based tools, as the district has focused on providing technology-based formative assessment tools to teachers. Because of implementation of technology-based tools being voluntary and autonomous, the factors which impacted the selection of applications and how learning was effected across sites were inconsistent.

In the case when consistent applications were chosen, the way in which they were infused varied across sites and teachers. One example is that some teacher participants utilize IXL to supplement learning activities, while others utilized the program for daily homework and practice. As such, all participants indicated a deficiency in teacher skill set relative to common technology-based tools. Teachers did not possess the same skills and training uniformly across sites. Concurrently, responses from all participants indicated a lack of adequate knowledge and training for teachers and students on how to utilize data from technology-based applications with fidelity to augment or modify instruction and learning experiences. This lack of fidelity did not allow for common data points within the process of collaboration or professional development. Furthermore, determining whether an application had an effect on student learning was largely dependent on which teacher participant was utilizing the program. Responses from teacher participants at one site regarding the effect of technology-based applications at times contradicted those made at other sites within the district. This was also the case regarding how data was collected, analyzed, and utilized to impact instruction. 
Ultimately, the variance in participant responses was directly related to the skills, abilities, and training relative to the program. As such, the voluntary nature of utilizing technology-based formative applications, in conjunction the varying levels of teacher competency, supported assertions participants that technology-based applications have the ability to impact learning more so than is currently being realized.

As noted by leader and specialist participants, teachers are not there yet. Leader, specialist, and principal participants conveyed a gap existed between the potential for technology-based tools to impact the learning cycle and what is actually being accomplished. Formative assessment feedback includes opportunities for clarification of goals, self-reflection and assessment, and collaboration, thus allowing teachers and students to identify and properly perceive gaps in learning, interpret the results, and make adjustments within learning activities (Biggs, 1998; Nicol \& Macfarlane-Dick, 2006; Rushton, 2005; Sadler, 1998; Vaughn et. al., 2013; Wiliam, 2011). Similarly, Nicol and Macfarlane-Dick (2006) and Wiliam (2011) proposed incorporating embedded formative assessment as a means to share teaching practices and provide feedback to students, both of which have a positive impact on student achievement. Within this research, the lack of consistency in which applications were utilized, a lack of common data points utilized for collaboration, and a lack of ability by teachers to analyze data to adjust instruction were evident barriers to maximizing student learning.

\section{Conclusions}

While past efforts of providing classrooms with technological tools such as computer workstations and Smart Boards increased the level of technology access, researchers (Jude et al., 2014; Wiliam, 2011) maintained initiatives in technology have 
failed to increase achievement as such efforts merely modified the method of providing equivalent instruction. Furthermore, researchers asserted that infusing technology in classrooms where teachers are trained to enhance feedback and modify instruction based on information gained should be further studied (Vaughn et al., 2013; Wiliam, 2011). This research sought to provide confirmation that teachers using technology-based formative assessment tools within the instructional cycle will further create conditions that promote student reflection on learning within and outside of the instructional space. Specifically, the purpose of this research was to determine if technology-based formative assessment applications impact student learning on specific learning standards. This study analyzed mobile learning opportunities, specific applications (IXL, ALEKS, and Schoology), and the quality and frequency of feedback to determine whether technologybased formative assessment applications provides data to inform instruction and learning.

Research from this study shows that teacher's utilization of technology-based formative assessment applications builds upon the principles of mobile learning theory, allowing students to receive feedback in a variety of ways not possible without technology. Students who utilize applications for remediation, proficiency and enrichment opportunities do so at greater rates, and with increased differentiation, when utilizing technology-based applications. Students obtain feedback in real-time, relative to the learning standard, and with immediate resources for explanation of deficiencies and remediation. Further results from this research conclude when technology-based formative assessment is utilized to augment or modify learning experiences, students have access to more information and learning experiences are personalized based on the students level of proficiency. 
This research also showed there is a difference in implementation of technologybased formative assessment across sites. These variances can be attributed to several contributing factors. The first factor contributing to the discrepancy of implementation is the lack of a consistent plan to infuse and embed technology-based formative assessment applications across district sites and classrooms. Although a few applications are supported (purchased) by the district, there are no expectations set forth by leadership that require teachers to use them. The type and frequency of use is an autonomous decision made by each teacher. In addition, even when teachers utilize the same applications, the research showed they do so inconsistently and with different purpose. As stated by researchers (Marzano, 2014; Rutheford, 2013; Wiliam, 2011), meaningful feedback provided as a function of peer collaboration, self-reflection, or instructor review has a positive impact on student learning. Despite not having a consistent plan across the district for embedding technology-based formative assessment applications, the research indicated each of the teacher participants have regularly infused and imbedded technology-based formative assessment within the instructional cycle of their classrooms. In addition, findings from this research show the infusion of technology-based formative assessment applications allows students to receive feedback on learning more frequently, often times immediately, which allows for remediation or enhancement of learning at an increased pace. Prior to technology-based feedback, participants indicated it could take several days for students to receive information regarding mastery of a learning standard. Further, prior technology-based applications, feedback was holistic rather than individualized and confined to context of the classroom setting. Technologybased feedback allows for personalized learning opportunities for students in real-time, 
with the ability to obtain remediation and assistance on learning targets in a non-teachercentered manner. Students can practice at an individualized level, receiving homework that is individual to them, when previously homework and practice was only differentiated if the teacher possessed the skills and knowledge to create individual homework assignments. Technology-based formative assessment, as evidenced in this research, increases or decreases the level of difficulty based on the current level of student understanding. Mobile learning enhances student learning by providing individualized access to information, immediacy in reflection, expedient and collaborative interaction, and convenience (Kukulska-Hulme, Sharples, Milrad, Arnedillo-Sanchez, \& Vavoula, 2009; Kynaslahti, 2003).

Lastly, participants within this study consistently referenced the full capacity of technology-based formative assessment applications to impact instruction, and thus student learning, has yet to be realized. Wiliam (2011) stated infusing technology alone would not yield the intended results of increased student achievement; however, the effective use of technology can be used to enhance formative assessment and positively impact student achievement and learning. Within this research, professional development with regard to technology tools and applications varies by site and teacher, and is highly dependent on teacher choice. Specifically, the research shows teacher participants received formal professional development training in applications such as IXL, ALEKS, and Schoology as well as other technology-based formative assessment tools. Although these trainings existed, the results of professional development did not yield a consistent implementation of strategies across learning environments. A successful plan for professional development, according to Wiliam \& Black (2009), 
dissertation in practice, B. Jacobs

develops skills in eliciting evidence of learning to provide feedback, adjusting future lessons and learning outcomes, and allowing students to use feedback to improve performance.

As a result, this research illustrates teacher participants within this research do not possess the same skills and abilities to adjust and adapt future learning experiences in a consistent way. Data collected from technology-based applications also varies by site. This also indicates collaboration and reflection opportunities are not impacted by technology-based formative assessment with regard to specific learning targets as there are no common data points to compare. As was observed in a teacher collaboration session, the extent to which technology-based tools was discussed was relative to how it would be embedded into the instructional unit. The embedding of technology in that circumstance was driven by ease of instruction, not increasing the amount of data related to the learning standard. As a result, the results of professional development relative to data collection and analysis in order to impact future learning experiences was inconsistent across participants, and limited to the extent of which teachers were willing to infuse within instructional practice. The results of this research indicate the infusion of the concept of technology-based formative assessment applications without a specific design for how they will be utilized, what data would be reviewed to reflect upon effectiveness, and what types of results would be analyzed to determine adjustments to instruction. Concurrently, teachers being provided autonomy to choose which technology-based formative assessment applications were utilized, without setting minimum implementation standards, resulted in a variety of responses by teachers with regard to the effectiveness of technology-based formative assessment applications. 


\section{Recommendations}

This study focused on highlighting the importance of embedding opportunities for technology-based formative assessement feedback within the teaching and learning cycle. The research revealed the timeliness and quality of feedback, as enhance by technology, impacted student learning in a positive capacity, and this inquiry supported that assertion. Following is the executive summary that will be presented to the district leadership team, instructional specialists, building leaders and mathematics teachers to enhance their understanding of how to technology can provide augmented or modified learning opportunities, as well as assist in developing a consistent district-wide plan for professional development that supports collaboration utilizing common data sets. In addition, the following executive summary highlights the importance technology in providing personalized, individualized, and immediate feedback to teachers and students on learning standards.

\section{Discussion}

While past efforts of providing classrooms with technological tools such as computer workstations and Smart Boards increased the level of technology access, researchers (Jude et al., 2014; Wiliam, 2011) maintained initiatives in technology have failed to increase achievement as such efforts merely modified the method of providing equivalent instruction. Furthermore, researchers asserted that infusing technology in classrooms where teachers are trained to enhance feedback and modify instruction based on information gained should be further studied (Vaughn et al., 2013; Wiliam, 2011). This research sought to provide confirmation that teachers using technology-based formative assessment tools within the instructional cycle will further create conditions 
that promote student reflection on learning within and outside of the instructional space.

Specifically, the purpose of this research was to determine if technology-based formative assessment applications impact student learning on specific learning standards. This study analyzed mobile learning opportunities, specific applications (IXL, ALEKS, and Schoology), and the quality and frequency of feedback to determine whether technologybased formative assessment applications provides data to inform instruction and learning.

Research from this study shows that teacher's utilization of technology-based formative assessment applications builds upon the principles of mobile learning theory, allowing students to receive feedback in a variety of ways not possible without technology. Students who utilize applications for remediation, proficiency and enrichment opportunities do so at greater rates, and with increased differentiation, when utilizing technology-based applications. Students obtain feedback in real-time, relative to the learning standard, and with immediate resources for explanation of deficiencies and remediation. Further results from this research conclude when technology-based formative assessment is utilized to augment or modify learning experiences, students have access to more information and learning experiences are personalized based on the students level of proficiency.

This research also showed there is a difference in implementation of technologybased formative assessment across sites. These variances can be attributed to several contributing factors. The first factor contributing to the discrepancy of implementation is the lack of a consistent plan to infuse and embed technology-based formative assessment applications across district sites and classrooms. Although a few applications are supported (purchased) by the district, there are no expectations set forth by leadership 
that require teachers to use them. The type and frequency of use is an autonomous decision made by each teacher. In addition, even when teachers utilize the same applications, the research showed they do so inconsistently and with different purpose. As stated by researchers (Marzano, 2014; Rutheford, 2013; Wiliam, 2011), meaningful feedback provided as a function of peer collaboration, self-reflection, or instructor review has a positive impact on student learning. Despite not having a consistent plan across the district for embedding technology-based formative assessment applications, the research indicated each of the teacher participants have regularly infused and imbedded technology-based formative assessment within the instructional cycle of their classrooms. In addition, findings from this research show the infusion of technology-based formative assessment applications allows students to receive feedback on learning more frequently, often times immediately, which allows for remediation or enhancement of learning at an increased pace. Prior to technology-based feedback, participants indicated it could take several days for students to receive information regarding mastery of a learning standard. Further, prior technology-based applications, feedback was holistic rather than individualized and confined to context of the classroom setting. Technologybased feedback allows for personalized learning opportunities for students in real-time, with the ability to obtain remediation and assistance on learning targets in a non-teachercentered manner. Students can practice at an individualized level, receiving homework that is individual to them, when previously homework and practice was only differentiated if the teacher possessed the skills and knowledge to create individual homework assignments. Technology-based formative assessment, as evidenced in this research, increases or decreases the level of difficulty based on the current level of 
student understanding. Mobile learning enhances student learning by providing individualized access to information, immediacy in reflection, expedient and collaborative interaction, and convenience (Kukulska-Hulme, Sharples, Milrad, Arnedillo-Sanchez, \& Vavoula, 2009; Kynaslahti, 2003).

Lastly, participants within this study consistently referenced the full capacity of technology-based formative assessment applications to impact instruction, and thus student learning, has yet to be realized. Wiliam (2011) stated infusing technology alone would not yield the intended results of increased student achievement, however, the effective use of technology can be used to enhance formative assessment and positively impact student achievement and learning. Within this research, professional development with regard to technology tools and applications varies by site and teacher, and is highly dependent on teacher choice. Specifically, the research shows teacher participants received formal professional development training in applications such as IXL, ALEKS, and Schoology as well as other technology-based formative assessment tools. Although these trainings existed, the results of professional development did not yield a consistent implementation of strategies across learning environments. A successful plan for professional development, according to Wiliam \& Black (2009), develops skills in eliciting evidence of learning to provide feedback, adjusting future lessons and learning outcomes, and allowing students to use feedback to improve performance.

As a result, this research illustrates teacher participants within this research do not possess the same skills and abilities to adjust and adapt future learning experiences in a consistent way. Data collected from technology-based applications also varies by site. 
This also indicates collaboration and reflection opportunities are not impacted by technology-based formative assessment with regard to specific learning targets as there are no common data points to compare. As was observed in a teacher collaboration session, the extent to which technology-based tools was discussed was relative to how it would be embedded into the instructional unit. The embedding of technology in that circumstance was driven by ease of instruction, not increasing the amount of data related to the learning standard. As a result, the results of professional development relative to data collection and analysis in order to impact future learning experiences was inconsistent across participants, and limited to the extent of which teachers were willing to infuse within instructional practice. The results of this research indicate the infusion of the concept of technology-based formative assessment applications without a specific design for how they will be utilized, what data would be reviewed to reflect upon effectiveness, and what types of results would be analyzed to determine adjustments to instruction. Concurrently, teachers being provided autonomy to choose which technology-based formative assessment applications were utilized, without setting minimum implementation standards, resulted in a variety of responses by teachers with regard to the effectiveness of technology-based formative assessment applications.

\section{Implications for Practice}

Based on the results of this study, districts cannot assume that immersing staff members in technology-based formative assessment strategies will have an impact on student learning. Utilizing such a method will infuse teachers with an abundance of resources, however, lacks the fidelity of how to utilize information from the applications to adjust the scope and sequence of learning. Furthermore, it is imperative that school 
districts adopt specific technology-based formative assessment applications, provide guided professional development to staff, and implement said applications with fidelity and oversight. Additionally, any district implementation plan should include the training of teachers to analyze data and provide training on how to use data when adjusting instruction to provide remediation to students when learning standards are not met. 


\section{Journal Article References}

Afshari, M., Bakar, K. A., Luan, W. S., Samah, B. A., \& Fooi, F. S. (2009). Factors affecting teachers' use of information and communication technology. International Journal of Instruction, 2(1), 77-104.

Amineh, R. J., \& Asl, H. D. (2015). Review of constructivism and social constructivism. Journal of Social Sciences, Literature and Languages, 1(1), 9-16. Anderson, T., \& Dron, J. (2011). Three generations of distance education pedagogy. The International Review of Research in Open and Distributed Learning, 12(3), 8097. doi: http://dx.doi.org/10.19173/irrodl.v12i3.890

Andersson, C., \& Palm, T. (2017). The impact of formative assessment on student achievement: a study of the effects of changes to classroom practice after a comprehensive professional development programme. Learning and Instruction, 49, 92-102. doi: https://doi.org/10.1016/j.learninstruc.2016.12.006

Baxter, P., \& Jack, S. (2008). Qualitative case study methodology: Study design and implementation for novice researchers. The Qualitative Report, 13(4), 544-559.

Burns, J.M. (1978). Leadership: Transformational leadership, transactional leadership. New York, NY: Harper \& Row.

Burns, M. K., Klingbeil, D. A., \& Ysseldyke, J. (2010). The effects of technologyenhanced formative evaluation on student performance on state accountability math tests. Psychology in the Schools, 47(6), 582-591. doi: 10.1002/pits.20492 Cochrane, T. (2012). Secrets of mlearning failures: Confronting reality. Research in Learning Technology, 20. Cochrane, T. (2016). Mobile VR in education: From 
dissertation in practice, B. Jacobs

the fringe to the mainstream. International Journal of Mobile and Blended Learning, 8(4), 44-60.

Conradie, P. W. (2014). Supporting self-directed learning by connectivism and personal learning environments. International Journal of Information and Education Technology, 4(3), 254-259. doi: http://dx.doi.org/10.7763/IJIET.2014.V4.408

Creswell, J. W. (2014). Research design: Qualitative, quantitative, and mixed methods approaches. Thousand Oaks, CA: Sage Publications, Inc.

Crompton, H. (2013). Mobile learning: New approach, new theory. In Berge, Z. L., \& Muilenburg, L. (Eds.). Handbook of mobile learning. (pp. 47-58). New York, NY: Routledge.

Crotty, M. (1998). The foundations of social research: Meaning and perspective in the research process. Thousand Oaks, CA: Sage Publications, Inc.

Dikko, M. (2016). Establishing construct validity and reliability: Pilot testing of a qualitative interview for research in Takaful (Islamic insurance). The Qualitative Report, 21(3), 521-528.

Doubleday, A. F., Brown, B., Patston, P. A., Jurgens-Toepke, P., Strotman, M. D., Koerber, A., ... \& Knight, G. W. (2015). Social constructivism and case-writing for an integrated curriculum. Interdisciplinary Journal of Problem-based Learning, 9(1), 9.

Dyson, L. E., \& Frawley, J. K. (2016). Mobile-Supported Fieldwork for First-Year Information Systems Students: A Way of Engaging Students in the Real World Context. In L. E. Dyson, W. Ng, \& J. Fergusson (Eds.) Mobile Learning FuturesSustaining Quality Research and Practice in Mobile Learning (pp. 349) 
dissertation in practice, B. Jacobs

Proceedings of the $15^{\text {th }}$ World Conference on Mobile and Contextual Learning, mlearn 2016. Sydney, [Australia].

Dyson, L. E., Litchfield, A., Raban, R., \& Tyler, J. (2009). Interactive classroom mLearning and the experiential transactions between students and lecturer. In Same places, different spaces (pp. 233 - 242), Proceedings of ascilite Auckland 2009. Sydney, [Australia].

Ekanayake, S. Y., \& Wishart, J. (2015). Integrating mobile phones into teaching and learning: A case study of teacher training through professional development workshops. British Journal of Educational Technology, 46(1), 173-189.

Fink, A. (2015). How to conduct surveys: A step-by-step guide. Thousand Oaks, CA; Sage Publications, Inc.

Flick, U. (2009). An introduction to qualitative research. Thousand Oaks, CA: Sage Publications, Inc.

Flick, U., Scott, W., \& Metzler, K. (2014). The SAGE handbook of qualitative data analysis. London, [England]: SAGE Publications, Ltd.

Gill, P., Stewart, K., Treasure, E., \& Chadwick, B. (2008). Methods of data collection in qualitative research: Interviews and focus groups. British dental journal, 204(6), 291-295.

Gold, R. L. (1958). Roles in sociological field observations. Social Forces, 36(3), 217223. Retrieved from http://doi.org.proxy.mul.missouri.edu/10.2307/2573808

Guest, G., MacQueen, K. M., \& Namey, E. E. (2012). Applied thematic analysis. Thousand Oaks, CA. Sage Publications.

Heitink, M. C., Van der Kleij, F. M., Veldkamp, B. P., Schildkamp, K., \& Kippers, W. B. 
dissertation in practice, B. Jacobs

(2016). A systematic review of prerequisites for implementing assessment for learning in classroom practice. Educational Research Review, 17, 50-62.

Kihoza, P., Zlotnikova, I., Bada, J., \& Kalegele, K. (2016). Classroom ICT integration in Tanzania: Opportunities and challenges from the perspectives of TPACK and SAMR models. International Journal of Education and Development Using Information and Communication Technology, 12(1), 107-128.

Kivunja, C. (2014). Do you want your students to be job-ready with 21 st century skills? change pedagogies: A pedagogical paradigm shift from Vygotskyian social constructivism to critical thinking, problem solving and Siemens' digital connectivism. International Journal of Higher Education, 3(3), 81.

Kizito, R. N. (2016). Connectivism in learning activity design: Implications for pedagogically-based technology adoption in African higher education contexts. The International Review of Research in Open and Distributed Learning, 17(2). 19-39. doi: http://dx.doi.org/10.19173/irrodl.v17i2.2217.

Kop, R., \& Hill, A. (2008). Connectivism: Learning theory of the future or vestige of the past?. The International Review of Research in Open and Distributed Learning, 9(3). doi: http://dx.doi.org/10.19173/irrodl.v9i3.523.

Kropf, D.C. (2013). Connectivism: 21st century's new learning theory. European Journal of Open, Distance and E-Learning, 16(2), 13-24.

Krueger, R. A., \& Casey, M. A. (2014). Focus groups: A practical guide for applied research. Thousand Oaks, CA: Sage publications, Inc. 
dissertation in practice, B. Jacobs

Kukulska-Hulme, A., Sharples, M., Milrad, M., Arnedillo-Sánchez, I., \& Vavoula, G. (2009). Innovation in mobile learning: A European perspective. International Journal of Mobile and Blended Learning (IJMBL), 1(1), 13-35.

Kynaslahti, H. (2003). In search of elements of mobility in the context of education. In H. Kynäslahti \& P. Seppälä (Eds.), Mobile learning (pp. 41-48). Helsinki, Finland: IT Press. Lincoln, Y. S., \& Guba, E. G. (1985). Naturalistic inquiry (Vol. 75). Newbury Park, CA: Sage Publications, Inc.

Lincoln, Y. S., Lynham, S. A., \& Guba, E. G. (2011). Paradigmatic controversies, contradictions, and emerging confluences, revisited. In N. K, Denzin \& Y. S. Lincoln (Eds.), The Sage handbook of qualitative research, 4, 97-128. Thousand Oaks, CA: Sage publications, Inc.

Magaña, S., \& Marzano, R. J. (2014). Using polling technologies to close feedback gaps. Educational Leadership, 71(6), (83-83).

Merriam, S. B., \& Tisdell, E. J. (2016). Qualitative research: A guide to design and implementation. San Francisco, CA: John Wiley \& Sons.

Nevo, B. (1985). Face validity revisited. Journal of Educational Measurement, 22(4), 287-293.

Pachler, N., Daly, C., Mor, Y., \& Mellar, H. (2010). Formative e-assessment: Practitioner cases. Computers \& Education, 54(3), 715-721.

Patton, M. Q. (2014). Qualitative research \& evaluation methods: Integrating theory and practice. Thousand Oaks, CA: Sage Publications, Inc.

Schunk, D. H. (2012). Learning theories an educational perspective ( $6^{\text {th }}$ ed.). Boston, MA: Pearson Education, Inc. 
dissertation in practice, B. Jacobs

Schutz, A. (1967). The phenomenology of the social world. Evanston, IL. Northwestern University Press.

Seidman, I. (2013). Interviewing as qualitative research: A guide for researchers in education and the social sciences ( $4^{\text {th }}$ Ed.). New York, NY. Teachers College Press.

Sharples, M., Taylor, J., \& Vavoula, G. (2007). A theory of learning for the mobile age. In R. Andrews \& C. Haythornthwaite (Eds.), The Sage handbook of e-learning research (pp. 221-247). Thousand Oaks, CA: Sage Publications Inc.

Tesch, R. (2013). Qualitative research: Analysis types and software. New York, NY: Routledge.

Van Manen, M. (2015). Researching lived experience: Human science for an action sensitive pedagogy ( $2^{\text {nd }}$ ed.). Walnut Creek, CA. Left Coast Press.

Vygotsky, L. S. (1978). Mind in society. Cambridge, MA: Harvard University Press. Wheeler, A. H., Goolkasian, P., Baird, A. C., \& Darden, B. V. (1999). Development of the neck pain and disability scale: Item analysis, face, and criterion-related validity. Spine, 24(13), 1290.

Wong, G. K. (2016). A new wave of innovation using mobile learning analytics for flipped classroom. In D. Churchill, J. L, T.K. Chiu, \& B. Fox (Eds.). Mobile Learning Design. Theories and Application (pp. 189-218). [Singapore]: Springer.

Xie, Y. (2016). On teaching reform of college English based on mobile learning. Revista Ibérica de Sistemas e Tecnologias de Informação, 9, 214-222. 
dissertation in practice, B. Jacobs

Yin, R. K. (2003). Case study research: Design and methods (3rd Ed.). Thousand Oaks, CA: Sage Publications, Inc. 


\section{SECTION SIX}

SCHOLARLY PRACTIONER REFLECTION 
dissertation in practice, B. Jacobs

I have found the process of completing this dissertation to be one of great reward. Through this process, I have met tremendous people within the cohort whom have influenced my learning in so many ways. First, and foremost, my advisor and mentor, Dr. Barbara Martin. Having her experience and expertise when reflecting on processes, findings, and ultimately the process of learning has been invaluable. When I started the process, Dr. Martin told us to pick something we are going to love reading, talking, listening and writing about for the next three years, and that was the best advice I could get. I am happy to say I never changed topics because of that comment, and my love for technology, feedback, and learning in mathematics guided my decision greatly in choosing this topic.

As for the ELPA program, specifically the EdD portion, I believe the process of a Dissertation In Practice encouraged and allowed me to dive into my own organization, something I might not have done otherwise. With the conclusions and results of my research, I was able to review a current practice and effect those I work with on a daily basis. In addition, I was able to expand my knowledge of district-wide practices through data triangulation, specifically affecting my own ability to impact learning. While I make efforts to encourage teachers to infuse technology within the teaching and learning process, I learned quickly that it is hard to transform current practices and expand the capabilities of others without a common and consistent plan. Ultimately, having a deeper understanding of formative assessment, as well as information related to specific technology-based applications, it will increase my ability to make informed decisions and impact learning within my career. 
Secondly, this process has allowed me to see how much we are truly asking of teachers within the teaching and learning process. Research on the effects of formative assessment feedback alone indicates the increased need to gather, analyze and apply information gained from feedback opportunities as frequently as possible to maximize gains in achievement. Although it seemed intuitive to state technology would enhance the learning process, this research uncovered that in mathematics it takes an immense amount of time, training, and preparation to infuse technology effectively. Further, for infusion processes to be effective, teachers must also be very knowledgeable about when technology infusion is necessary. Hearing there are times, technology does not provide enough information as compared to traditional strategies was eye opening. Realizing how much emphasis middle school mathematics teacher's focus on the depth of learning processes was a vital component to the study.

Lastly, I am a better person. I have been able to learn how to deal with stress, overload, and frankly learn how to deal with something that seemed at one point like it was never going to go away. When entering this program, I really held the opinion that I was a good writer. After submitting papers then having them come back completely ripped apart, it really changes your thinking before you hit submit. I now see writing differently, and often correct myself before I even send it in. Also, categorizing and storing information to utilize it JUST when it is needed was never something I considered being my strength. I have learned, as a part of this process, how to select books, articles and resources that cover a variety of applicable topics. One reason being that when a source holds more information and research, I found it to be more credible throughout data collection. As such, selecting and organizing resources to support conclusions is 
now something I value as a strength. Ultimately, I value what I have learned from this process and look forward to applying the skills and knowledge acquired to my career and life. 


\section{References}

Afshari, M., Bakar, K. A., Luan, W. S., Samah, B. A., \& Fooi, F. S. (2009). Factors affecting teachers' use of information and communication technology. International Journal of Instruction, 2(1), 77-104.

Ally, M. (2004). Foundations of educational theory for online learning. Theory and Practice of Online Learning, 2, 15-44.

Alismail, H. A., \& McGuire, P. (2015). 21st century standards and curriculum: Current research and practice. Journal of Education and Practice, 6(6), 150-154.

Amineh, R. J., \& Asl, H. D. (2015). Review of constructivism and social constructivism. Journal of Social Sciences, Literature and Languages, 1(1), 9-16.

Anderson, T., \& Dron, J. (2011). Three generations of distance education pedagogy. The International Review of Research in Open and Distributed Learning, 12(3), 8097. doi: http://dx.doi.org/10.19173/irrodl.v12i3.890

Andersson, C., \& Palm, T. (2017). The impact of formative assessment on student achievement: a study of the effects of changes to classroom practice after a comprehensive professional development programme. Learning and Instruction, 49, 92-102. doi: https://doi.org/10.1016/j.learninstruc.2016.12.006

Alam, I. (2015). Teachers' classroom behavior and its impacts upon learning process. Asian Journal of Technology \& Management Research, 5(02). 19-24.

Avolio, B. J., \& Bass, B. M. (1995). Individual consideration viewed at multiple levels of analysis: A multi-level framework for examining the diffusion of transformational leadership. The Leadership Quarterly, 6(2), 199-218. doi: https://doi.org/10.1016/1048-9843(95)90035-7 
dissertation in practice, B. Jacobs

Barry, W. (2013). Connectivism: Theory or phenomenon? The Accidental Technologist. Retrieved from http://www.waynebarry.com/2013/04/29/connectivism-theory-orphenomenon/

Bass, B. M., \& Avolio, B. J. (1990). Transformational leadership development: Manual for the multifactor leadership questionnaire. Palo Alto, CA: Consulting Psychologists Press.

Bass, B. M., \& Riggio, R. E. (2006). Transformational leadership (2 ${ }^{\text {nd }}$ ed.). Mahwah, NJ: Lawrence Erlbaum Associates, Inc.

Baxter, P., \& Jack, S. (2008). Qualitative case study methodology: Study design and implementation for novice researchers. The Qualitative Report, 13(4), 544-559.

Beatty, I. D., \& Gerace, W. J. (2009). Technology-enhanced formative assessment: A research-based pedagogy for teaching science with classroom response technology. Journal of Science Education and Technology, 18(2), 146-162.

Bebell, D., Russell, M., \& O’Dwyer, L. (2004). Measuring teachers’ technology uses: why multiple-measures are more revealing. Journal of Research on Technology in Education, 37, 45-63. doi: 10.1080/15391523.2004.10782425.

Bell, F. (2011). Connectivism: Its place in theory-informed research and innovation in technology-enabled learning. The International Review of Research in Open and Distributed Learning, 12(3), 98-118.

Bennett, R. E., \& Gitomer, D. H. (2009). Transforming K-12 assessment: Integrating accountability testing, formative assessment and professional support. In C. Wyatt-Smith \& J. Cumming (Eds.), Assessment issues of the 21st Century (pp. 4361). New York: Springer Publishing Company. 
dissertation in practice, B. Jacobs

Bennis, W., \& Nanus, B. (1985). The strategies for taking charge. Leaders. New York, NY. Harper. Row.

Berliner, D. C. (2006). Educational psychology: Searching for essence throughout a century of influence. Handbook of educational psychology, 2, 3-42.

Bhagat, K. K., \& Spector, J. M. (2017). Formative assessment in complex problemsolving domains: The emerging role of assessment technologies. Journal of Educational Technology \& Society, 20(4), 312-317.

Biggs, J. (1998). Assessment and classroom learning: a role for summative assessment?. Assessment in Education: Principles, Policy \& Practice, 5(1), 103110.

Black, P., \& Wiliam, D. (1998). Assessment and classroom learning. Assessment in Education, 5(1), 7-74.

Black, P., \& Wiliam, D. (2009). Developing the theory of formative assessment. Educational Assessment, Evaluation and Accountability (formerly Journal of Personnel Evaluation in Education), 21(1), 5-31.

Blanchard, K., Zigarmi, D., \& Zigarmi, P. (1985). Leadership and the one minute manager: Increasing effectiveness through situational leadership. New York, NY: William Morrow and Company.

Boghossian, P. (2006). Behaviorism, constructivism, and socratic pedagogy. Educational Philosophy and Theory, 38(6), 713-722.

Bolman, L. G., \& Deal, T. E. (2013). Reframing organizations: Artistry, choice, \& leadership (5 ${ }^{\text {th }}$ ed.). San Francisco, CA: Jossey-Bass.

Bolman, L. G., \& Deal, T. E. (2010). Reframing the path to school leadership: A guide 
dissertation in practice, B. Jacobs

for teachers and principals ( $2^{\text {nd }}$ ed.). Thousand Oaks, CA: Corwin Press.

Bransford, J., Brown, A., \& Cocking, R. (Eds.). (2004). How people learn: Brain, mind, experience, and school: Expanded edition. Washington, DC: National Academy Press

Burns, J.M. (1978). Leadership: Transformational leadership, transactional leadership. New York, NY: Harper \& Row.

Burns, M. K., Klingbeil, D. A., \& Ysseldyke, J. (2010). The effects of technologyenhanced formative evaluation on student performance on state accountability math tests. Psychology in the Schools, 47(6), 582-591. doi: 10.1002/pits.20492

Burston, J. (2015). Review of mobile learning: languages, literacies, and cultures. Language Learning and Technology, 19(2), 44-49. Retrieved from http://ktisis.cut.ac.cy/bitstream/10488/9401/1/Burston.pdf.

Carrejo, D., Cortez, T., \& Reinhartz, J. (2010). Exploring principal leadership roles within a community of practice to promote science performance of English language learners. Academic Leadership Journal, 8(4), 9-23.

Chang, K. E., Wu, L. J., Weng, S. E., \& Sung, Y. T. (2012). Embedding game-based problem-solving phase into problem-posing system for mathematics learning. Computers \& Education, 58(2), 775-786.

Chappuis, J. (2015). Seven strategies of assessment for learning. ( $2^{\text {nd }}$ ed.) Portland, OR: Pearson Education, Inc.

Charmaz, K. (2006). Constructing grounded theory. Thousand Oaks, CA: Sage Publications, Inc. 
dissertation in practice, B. Jacobs

Chee, K. N., Yahaya, N., Ibrahim, N. H., \& Hasan, M. N. (2017). Review of mobile learning trends 2010-2015: A meta-analysis. Journal of Educational Technology \& Society, 20(2), 113-126.

Cheon, J., Lee, S., Crooks, S. M., \& Song, J. (2012). An investigation of mobile learning readiness in higher education based on the theory of planned behavior. Computers \& Education, 59(3), 1054-1064.

Cheung, A. C., \& Slavin, R. E. (2013). The effectiveness of educational technology applications for enhancing mathematics achievement in K-12 classrooms: A meta-analysis. Educational Research Review, 9, 88-113.

Cho, K. (2005). When multi-peers give better advice than the expert: The type and impact of feedback given by students and an expert on student writing (Doctoral dissertation, University of Pittsburg). Dissertation Abstracts International. 65 (10).

Cochrane, T. (2012). Secrets of mlearning failures: Confronting reality. Research in Learning Technology, 20.

Cochrane, T. (2016). Mobile VR in education: From the fringe to the mainstream. International Journal of Mobile and Blended Learning, 8(4), 44-60.

Conejo, R., Garcia-Viñas, J. I., Gastón, A., \& Barros, B. (2016). Technology-enhanced formative assessment of plant identification. Journal of Science Education and Technology, 25(2), 203-221.

Cope, B., \& Kalantzis, M. (2009). Ubiquitous learning: An agenda for educational transformation. In Bill Cope, \& Mary Kalantzis (Eds.), Ubiquitous learning. Champaign, IL: University of Illinois Press. 
Conradie, P. W. (2014). Supporting self-directed learning by connectivism and personal learning environments. International Journal of Information and Education Technology, 4(3), 254-259. doi: http://dx.doi.org/10.7763/IJIET.2014.V4.408

Corbeil, J. R., \& Valdes-Corbeil, M. E. (2007). Are you ready for mobile learning?. Educause Quarterly, 30(2), 51.

Cornelius, S., \& Marston, P. (2009). Towards an understanding of the virtual context in mobile learning. ALT-J, 17(3), 161-172. doi: 10.1080/09687760903247617

Cowie, B., \& Bell, B. (1999). A model of formative assessment in science education. Assessment in Education: Principles, Policy \& Practice, 6(1), 101-116.

Cox, E. (2015). Coaching and adult learning: Theory and practice. New Directions for Adult and Continuing Education, 2015(148), 27-38.

Creswell, J. W. (2014). Research design: Qualitative, quantitative, and mixed methods approaches. Thousand Oaks, CA: Sage Publications, Inc.

Crompton, H. (2013). Mobile learning: New approach, new theory. In Berge, Z. L., \& Muilenburg, L. (Eds.). Handbook of mobile learning. (pp. 47-58). New York, NY: Routledge.

Crotty, M. (1998). The foundations of social research: Meaning and perspective in the research process. Thousand Oaks, CA: Sage Publications, Inc.

Czaja, S. J., Charness, N., Fisk, A. D., Hertzog, C., Nair, S. N., Rogers, W. A., \& Sharit, J. (2006). Factors predicting the use of technology: Findings from the center for research and education on aging and technology enhancement (CREATE). Psychology and aging, 21(2), 333.

Dansereau Jr, F., Graen, G., \& Haga, W. J. (1975). A vertical dyad linkage approach to 
dissertation in practice, B. Jacobs

leadership within formal organizations: A longitudinal investigation of the role making process. Organizational behavior and human performance, 13(1), 46-78.

Department of Elementary and Secondary Education (2018). District Report Card.

Retrieved from https://mcds.dese.mo.gov/quickfacts/SitePages/District

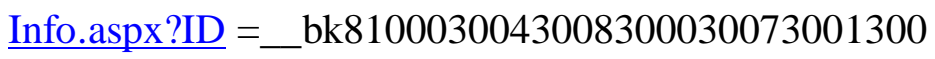

Department of Elementary and Secondary Education (2019). College and Career

Readiness. Retrieved from https://dese.mo.gov/college-career-

readiness/curriculum/mathematics

Dikko, M. (2016). Establishing construct validity and reliability: Pilot testing of a qualitative interview for research in Takaful (Islamic insurance). The Qualitative Report, 21(3), 521-528.

DuFour, R., \& Eaker, R. (1998). Professional Learning Communities at Work: Best Practices for Enhancing Students Achievement. Bloomington, IN: Solution Tree Press.

Dionne, S. D., Gupta, A., Sotak, K. L., Shirreffs, K. A., Serban, A., Hao, C., ... \& Yammarino, F. J. (2014). A 25-year perspective on levels of analysis in leadership research. The Leadership Quarterly, 25(1), 6-35.

Doubleday, A. F., Brown, B., Patston, P. A., Jurgens-Toepke, P., Strotman, M. D., Koerber, A., ... \& Knight, G. W. (2015). Social constructivism and case-writing for an integrated curriculum. Interdisciplinary Journal of Problem-based Learning, 9(1), 9.

Downes, S. (2008). Places to go: Connectivism \& connective knowledge. Innovate: Journal of Online Education, 5(1), 6. 
dissertation in practice, B. Jacobs

Driscoll, M. P. (2005). Psychology of learning for instruction. Boston, MA: Pearson Education, Inc.

Duijn, C. C., Welink, L. S., Mandoki, M., ten Cate, O. T., Kremer, W. D., \& Bok, H. G. (2017). Am I ready for it? Students' perceptions of meaningful feedback on entrustable professional activities. Perspectives on medical education, 6(4), 256264.

Dunaway, M. K. (2011). Connectivism: Learning theory and pedagogical practice for networked information landscapes. Reference services review, 39(4), 675-685.

Dunn, K. E., \& Mulvenon, S. W. (2009). A critical review of research on formative assessment: The limited scientific evidence of the impact of formative assessment in education. Practical Assessment, Research \& Evaluation, 14(7), 1-11.

Dyson, L. E., \& Frawley, J. K. (2016). Mobile-Supported Fieldwork for First-Year Information Systems Students: A Way of Engaging Students in the Real World Context. In L. E. Dyson, W. Ng, \& J. Fergusson (Eds.) Mobile Learning FuturesSustaining Quality Research and Practice in Mobile Learning (pp. 349) Proceedings of the $15^{\text {th }}$ World Conference on Mobile and Contextual Learning, mlearn 2016. Sydney, [Australia].

Dyson, L. E., Litchfield, A., Raban, R., \& Tyler, J. (2009). Interactive classroom mLearning and the experiential transactions between students and lecturer. In Same places, different spaces (pp. 233 - 242), Proceedings of ascilite Auckland 2009. Sydney, [Australia]. 
dissertation in practice, B. Jacobs

Ekanayake, S. Y., \& Wishart, J. (2015). Integrating mobile phones into teaching and learning: A case study of teacher training through professional development workshops. British Journal of Educational Technology, 46(1), 173-189.

Erdogan, B., \& Bauer, T. N. (2014). Leader-member exchange (LMX) theory: The relational approach to leadership. In D. V. Day The Oxford handbook of leadership and organizations (pp. 407). New York, NY. The Oxford University Press.

Ertmer, P. A., \& Newby, T. J. (2013). Behaviorism, cognitivism, constructivism: Comparing critical features from an instructional design perspective. Performance Improvement Quarterly, 26(2), 43-71.

Eyyam, R., \& Yaratan, H. S. (2014). Impact of use of technology in mathematics lessons on student achievement and attitudes. Social Behavior and Personality: An International Journal, 42(1), 31S-42S.

Faber, J. M., Luyten, H., \& Visscher, A. J. (2017). The effects of a digital formative assessment tool on mathematics achievement and student motivation: Results of a randomized experiment. Computers \& Education, 106, 83-96.

Feldman, A., \& Capobianco, B. M. (2008). Teacher learning of technology enhanced formative assessment. Journal of Science Education and Technology, 17(1), 8299.

Fenstermacher, G. D., \& Richardson, V. (2005). On making determinations of quality in teaching. Teachers College Record, 107(1), 186-213.

Fink, A. (2015). How to conduct surveys: A step-by-step guide. Thousand Oaks, CA; Sage Publications, Inc. 
dissertation in practice, B. Jacobs

Flick, U. (2009). An introduction to qualitative research. Thousand Oaks, CA: Sage Publications, Inc.

Flick, U., Scott, W., \& Metzler, K. (2014). The SAGE handbook of qualitative data analysis. London, [England]: SAGE Publications, Ltd.

Fowler, F.C. (2004). Policy studies for educational leaders: an introduction (2nd ed.). Upper Saddle River, NJ: Pearson.

Gentles, S. J., Charles, C., Ploeg, J., \& McKibbon, K. A. (2015). Sampling in qualitative research: Insights from an overview of the methods literature. The Qualitative Report, 20(11), 1772.

Grant, M. M., Tamim, S., Brown, D. B., Sweeney, J. P., Ferguson, F. K., \& Jones, L. B. (2015). Teaching and learning with mobile computing devices: Case study in K12 classrooms. TechTrends, 59(4), 32-45.

Gill, P., Stewart, K., Treasure, E., \& Chadwick, B. (2008). Methods of data collection in qualitative research: Interviews and focus groups. British dental journal, 204(6), 291-295.

Gold, R. L. (1958). Roles in sociological field observations. Social Forces, 36(3), 217223. Retrieved from http://doi.org.proxy.mul.missouri.edu/10.2307/2573808 Guest, G., MacQueen, K. M., \& Namey, E. E. (2012). Applied thematic analysis. Thousand Oaks, CA. Sage Publications.

Hamilton, E. R., Rosenberg, J. M., \& Akcaoglu, M. (2016). The substitution augmentation modification redefinition (SAMR) model: A critical review and use. TechTrends, 60(5), 433-441. doi: 10.1007/s11528-016-0091-y

Harasim, L. (2012). Learning theory and online technologies. New York, NY: Routledge. 
dissertation in practice, B. Jacobs

Hattie, J. (2012). Visible learning for teachers: Maximizing impact on learning. New York, NY: Routledge.

Heitink, M. C., Van der Kleij, F. M., Veldkamp, B. P., Schildkamp, K., \& Kippers, W. B. (2016). A systematic review of prerequisites for implementing assessment for learning in classroom practice. Educational Research Review, 17, 50-62.

Hemphill, J. K. (1949). Situational factors in leadership. Ohio State University. Bureau of Educational Research Monograph, 32(xii), 1-136.

Herman, A. M. (1999). Futurework: Trends and challenges for work in the 21st century. Washington, DC: U.S. Department of Labor

Hernandez, F., \& McKenzie, K. B. (2010). Resisting social justice in leadership preparation programs: Mechanisms that subvert. Journal of Research on Leadership Education, 5(3.2), 48-72. Doi: https://doi.org/10.1177/194277511000500302. Hudson, T. (2014). Best Practices for Evaluating Digital Curricula. DreamBox Learning, Inc. Retrieved November, 16, 2018 from https://fs24.formsite.com/ edweek/images/WP-DreamBox-Best-Practices-for-Evaluating-DigitalCurricula.pdf

Hussain, H. K., Talib, N. A., \& Shah, I. M. (2014). Exploring the impact of transformational leadership on process innovation and product innovation: A Case of Iraqi Public Universities. Asian Social Science, 10(21), 168.

Hwang, G. J., \& Chang, H. F. (2011). A formative assessment-based mobile learning approach to improving the learning attitudes and achievements of students. Computers \& Education, 56(4), 1023-1031. 
dissertation in practice, B. Jacobs

Hwang, G. J., \& Tsai, C. C. (2011). Research trends in mobile and ubiquitous learning: A review of publications in selected journals from 2001 to 2010. British Journal of Educational Technology, 42(4), E65-E70. doi: 10.1111/j.14678535.2011.01183.x.

Inan, F. A., \& Lowther, D. L. (2010). Laptops in the K-12 classrooms: Exploring factors impacting instructional use. Computers \& Education, 55(3), 937-944. International Society for Technology Education (2016). ISTE Standards for Students. Retrieved from https://www.iste.org/standards/forstudents\#startstandards.

International Society of Technology Educators. (2018). Journal of Research on Technology in Education. Retrieved from http://www.tandfonline.com/action/authorSubmission? journalCode $=$ cher20\&page $=$ instructions.

Jacobs-Israel, M., \& Moorefield-Lang, H. (2013). Redefining technology in libraries and schools: AASL best apps, best websites, and the SAMR model. Teacher Librarian, 41(2), 16.

Johnson, A. G. (2006). Privilege, power, and difference (2nd ed.). Boston, MA: McGraw-Hill.

Johnson, G. M. (2014). The ecology of interactive learning environments: Situating traditional theory. Interactive Learning Environments, 22(3), 298-308.

Jude, L. T., Kajura, M. A., \& Birevu, M. P. (2014). Adoption of the SAMR model to asses ICT pedagogical adoption: A case of Makerere University. International Journal of e-Education, e-Business, e-Management and e-Learning, 4(2), 106. 
dissertation in practice, B. Jacobs

Judge, T. A., Bono, J. E., Ilies, R., \& Gerhardt, M. W. (2002). Personality and leadership: a qualitative and quantitative review. Journal of Applied Psychology, 87(4), 765.

Kihoza, P., Zlotnikova, I., Bada, J., \& Kalegele, K. (2016). Classroom ICT integration in Tanzania: Opportunities and challenges from the perspectives of TPACK and SAMR models. International Journal of Education and Development Using Information and Communication Technology, 12(1), 107-128.

Kirschner, P. A. (2004). Design, development, and implementation of electronic learning environments for collaborative learning. Educational Technology Research and Development, 52(3), 39-46.

Kivunja, C. (2014). Do you want your students to be job-ready with 21 st century skills? change pedagogies: A pedagogical paradigm shift from Vygotskyian social constructivism to critical thinking, problem solving and Siemens' digital connectivism. International Journal of Higher Education, 3(3), 81.

Kizito, R. N. (2016). Connectivism in learning activity design: Implications for pedagogically-based technology adoption in African higher education contexts. The International Review of Research in Open and Distributed Learning, 17(2). 19-39. doi: http://dx.doi.org/10.19173/irrodl.v17i2.2217.

Kop, R., \& Hill, A. (2008). Connectivism: Learning theory of the future or vestige of the past?. The International Review of Research in Open and Distributed Learning, 9(3). doi: http://dx.doi.org/10.19173/irrodl.v9i3.523.

Kotter, J. P. (1990). How leadership differs from management. New York: Free Press, 240, 59-68.

Kposowa, A. J., \& Valdez, A. D. (2013). Student laptop use and scores on standardized 
dissertation in practice, B. Jacobs

tests. Journal of Educational Computing Research, 48(3), 345-379.

Kropf, D.C. (2013). Connectivism: 21st century's new learning theory. European Journal of Open, Distance and E-Learning, 16(2), 13-24.

Krueger, R. A., \& Casey, M. A. (2014). Focus groups: A practical guide for applied research. Thousand Oaks, CA: Sage publications, Inc.

Kukulska-Hulme, A., Sharples, M., Milrad, M., Arnedillo-Sánchez, I., \& Vavoula, G. (2009). Innovation in mobile learning: A European perspective. International Journal of Mobile and Blended Learning (IJMBL), 1(1), 13-35.

Kynaslahti, H. (2003). In search of elements of mobility in the context of education. In H. Kynäslahti \& P. Seppälä (Eds.), Mobile learning (pp. 41-48). Helsinki, Finland: IT Press.

LeCompte, M. D., \& Schensul, J. J. (2010). Designing \& conducting ethnographic research: An introduction ( $2^{\text {nd }}$ ed.). Lanham, MD: Alta Mira Press.

Lee, H., Feldman, A., \& Beatty, I. D. (2012). Factors that affect science and mathematics teachers' initial implementation of technology-enhanced formative assessment using a classroom response system. Journal of Science Education and Technology, 21(5), 523-539.

Lees Summit School District (2016). Comprehensive School Improvement Plan: Destination 2021. Retrieved April 22, 2016, from http://cdn.lsr7.org/wpcontent/uploads/2012/08/ CSIP -2014-15-Revision.pdf.

Lees Summit School District (2018). History - 1949-Today. Retrieved from https://lsr7.org/district/about-us/history-from-1949-to-today/

Lei, J., \& Zhao, Y. (2007). Technology uses and student achievement: A longitudinal 
dissertation in practice, B. Jacobs

study. Computers \& Education, 49(2), 284-296.

Lencioni, P. (2002). The five dysfunctions of a team: A leadership fable. San Francisco, CA: Jossey-Bass.

Levi, D. J. (2014). Group dynamics for teams (4th ed.). Los Angeles, CA: Sage Publications, Inc.

Liden, R. C., Sparrowe, R. T., \& Wayne, S. J. (1997). Leader-member exchange theory: The past and potential for the future. Research in Personnel and Human Resources Management, 15, 47-120.

Lincoln, Y. S., \& Guba, E. G. (1985). Naturalistic inquiry (Vol. 75). Newbury Park, CA: Sage Publications, Inc.

Lincoln, Y. S., Lynham, S. A., \& Guba, E. G. (2011). Paradigmatic controversies, contradictions, and emerging confluences, revisited. In N. K, Denzin \& Y. S.

Lincoln (Eds.), The Sage handbook of qualitative research, 4, 97-128. Thousand Oaks, CA: Sage publications, Inc.

Looi, C. K., Wong, L. H., So, H. J., Seow, P., Toh, Y., Chen, W., Zhang, B., Norris, C., \& Soloway, E. (2009). Anatomy of a mobilized lesson: Learning my way. Computers \& Education, 53(4), 1120-1132.

Luis, R., \& D'Cunha, T. (2014). The role, essence and contributions of educational psychology to the field of education. International Journal of Education and Management Studies, 4(4), 370.

Lynch, Z., \& Sankey, M. (2016). Reboot your course-from beta to better. In L.E. Dyson, W. Ng, \& J. Fergusson (Eds.) Mobile Learning Futures-Sustaining Quality Research and Practice in Mobile Learning, Proceedings of the World Conference 
dissertation in practice, B. Jacobs

on Mobile and Contextual Learning (mLearn 2016), Sydney, 173-182. Retrieved November 4, 2018 from https://eprints.usq.edu.au/29911/1/M_Learn_ 2016_paper.pdf.

MacCallum, K., \& Parsons, D. (2016). A theory-ology of mobile learning:

Operationalizing learning theories with mobile activities. Proceedings of the World Conference on Mobile and Contextual Learning (mLearn 2016), Sydney, 173-182. Retrieved from http://iamlearn.org/wp-content/uploads/Proceedings_ MLearn2016Final.pdf

Magaña, S., \& Marzano, R. J. (2014). Using polling technologies to close feedback gaps. Educational Leadership, 71(6), (83-83).

Manning, K. (2017). Organizational theory in higher education. New York, NY: Routledge.

Marshall, C., \& Rossman, G. B. (2014). Designing qualitative research. Thousand Oaks, CA: Sage publications, Inc.

Martin, B. (2015). Successful implementation of TPACK in teacher preparation programs. International Journal on Integrating Technology in Education (IJITE), 4(1), 17-26.

Marzano, R. J. (2006). Classroom assessment \& grading that work. Alexandria, VA: ASCD.

McCleskey, J. A. (2014). Situational, transformational, and transactional leadership and leadership development. Journal of Business Studies Quarterly, 5(4), 117.

Merriam, S. B., \& Tisdell, E. J. (2016). Qualitative research: A guide to design and implementation. San Francisco, CA: John Wiley \& Sons. 
Mikelic, K. K., Lipicnik, B., \& Tekavcic, M. (2010). Ethical Leadership. International Journal of Management \& Information Systems, 14(5), 31-41.

Mintzberg, H. (2005). The five basic parts of the organization. In J. M. Shafritz, J. S. Ott, \& Y. S. Jang (Eds.), Classics of organization theory (6th ed.) (pp. 219-230). Belmont, CA: Wadsworth. (Reprinted from The structure of organizations: A synthesis of research, pp. 18-34, by H. Mintzberg, Ed., 1979, Upper Saddle River, NJ: Prentice-Hall).

Moore, B. V. (1927). The May conference on leadership. Personnel Journal, 6(124), 5074

Neebe, D. \& Roberts, J. (2015). Power Up: Making the shift to 1:1 teaching and learning Portland, ME: Stenhouse Publishers.

Nemanich, L. A., \& Keller, R. T. (2007). Transformational leadership in an acquisition: A field study of employees. The Leadership Quarterly, 18(1), 49-68.

Nevo, B. (1985). Face validity revisited. Journal of Educational Measurement, 22(4), 287-293.

Nicol, D. J., \& Macfarlane-Dick, D. (2006). Formative assessment and self-regulated learning: A model and seven principles of good feedback practice. Studies in Higher Education, 31(2), 199-218.

Niederhauser, D. S., \& Lindstrom, D. L. (2018). Instructional technology integration models and frameworks: Diffusion, competencies, attitudes, and dispositions. In J

Voogt, G. Knezek, R. Christensen, \& KW Lai (Eds.). Handbook of Information Technology in Primary and Secondary Education (pp. 1-21). [Cham]; Springer. doi: https://doi.org/10.1007/978-3-319-53803-7_23-1. 
dissertation in practice, B. Jacobs

Northouse, P. G. (2015). Leadership: Theory and practice. Thousand Oaks, CA. Sage Publications, Inc.

Offer, J., \& Bos, B. (2009). The design and application of technology-based courses in the mathematics classroom. Computers \& Education, 53(4), 1133-1137.

Ogata, H., \& Yano, Y. (2004). Context-aware support for computer-supported ubiquitous learning. Proceedings of IEEE International Workshop on Wireless \& Mobile Technologies in Education (WMTE 2004), March 25, 2004 (pp. 27-34). JhongLi, Taiwan: IEEE Computer Society. doi:10.1109/WMTE.2004. 1281330.

Olmanson, J. D., Abebe, F. F., Jones, V., Kyle, E., Lucas, L., Robbins, K., \& Liu, X. (2015). Caught in the tractor beam of larger influences: The filtration of innovation in education technology design. World Academy of Science, Engineering and Technology, International Journal of Social, Behavioral, Educational, Economic, Business and Industrial Engineering, 9(7), 2268-2272.

Ormrod, J. (2012). Human learning (6th ed.). New Jersey, NY: Pearson Education, Inc. O'Sullivan, M. K., \& Dallas, K. B. (2017). A collaborative approach to implementing 21 st century skills in a high school senior research class. Education Libraries, 33(1), 3-9.

Ozan O, Kesim M (2011). Providing scaffolding by using mobile applications in connectivist learning environment. Retrieved from http://www.ingedewaard. net/papers/connectivism/2008_Ozan_Kesim_ProvidingScaffolding_by_UsingMo bileApplications_in_ConnectivistLearningEnvironment.pdf.

Pachler, N., Bachmair, B., \& Cook, J. (2011). Mobile learning: Structures, agency, practices. New York, NY: Springer. 
dissertation in practice, B. Jacobs

Pachler, N., Daly, C., Mor, Y., \& Mellar, H. (2010). Formative e-assessment: Practitioner cases. Computers \& Education, 54(3), 715-721.

Pahomov, L. (2014). Authentic learning in the digital age: Engaging students through inquiry. Alexandria, VA. ASCD.

Park, Y. (2011). A pedagogical framework for mobile learning: Categorizing educational applications of mobile technologies into four types. The International Review of Research in Open and Distributed Learning, 12(2), 78-102.

Parsons D, Wishart J, Thomas H (2016) Exploring Mobile Affordances in the Digital Classroom. In I. Arnedillo-Sanchez \& P. Isaias (Eds.) Proceedings of 12th International Conference on Mobile Learning (Mobile Learning 2016). IADIS. 43-50.

Patton, M. Q. (2008). Utilization-focused evaluation. (4 ${ }^{\text {th }}$ ed.). Thousand Oaks, CA. Sage Publications, Inc.

Patton, M. Q. (2015). Qualitative research \& evaluation methods: Integrating theory and practice. Thousand Oaks, CA: Sage Publications, Inc.

Peters, K. (2007). m-Learning: Positioning educators for a mobile, connected future. The International Review of Research in Open and Distributed Learning, 8(2).

Pfeffer, J. (1992). Managing with power: Politics and influence in organizations. Boston, MA: Harvard Business School Press.

Pfeiffer, V. D., Gemballa, S., Jarodzka, H., Scheiter, K., \& Gerjets, P. (2009). Situated learning in the mobile age: Mobile devices on a field trip to the sea. ALT-J, 17(3), 187-199. 
Pilli, O., \& Aksu, M. (2013). The effects of computer-assisted instruction on the achievement, attitudes and retention of fourth grade mathematics students in North Cyprus. Computers \& Education, 62, 62-71.

Puentedura, R. R. (2013). SAMR: Moving from enhancement to transformation [Web log post]. Retrieved from http://www.hippasus.com/rrpweblog/archives/ 000095.html.

Quinton, S. R., \& Allen, M. M. (2014). The social processes of web 2.0 collaboration: Towards a new model for virtual learning. In M. Gosper, \& D. Ifenthaler (Eds.). Curriculum models for the 21 st century (pp. 35-53). New York, NY: Springer.

Railean, E. A. (2017). Impacts of digital revolution on learning. In E. Railean (Eds.) User Interface Design of Digital Textbooks: How Screens Affect Learning (pp. 1-22). [Singapore]: Springer.

Reeves, J. L., Gunter, G. A., \& Lacey, C. (2017). Mobile learning in pre-kindergarten: Using student feedback to inform practice. Educational Technology \& Society, 20(1), 37-44.

Romrell, D., Kidder, L. C., \& Wood, E. (2014). The SAMR model as a framework for evaluating mLearning. Online Learning Journal, 18(2). Retrieved from https://www.learntechlib.org/p/183753/.

Rowold, J., \& Heinitz, K. (2007). Transformational and charismatic leadership: Assessing the convergent, divergent and criterion validity of the MLQ and the CKS. The Leadership Quarterly, 18(2), 121-133.

Rushton, A. (2005). Formative assessment: A key to deep learning? Medical teacher, 27(6), 509-513. 
dissertation in practice, B. Jacobs

Rutherford, M. (2013). The artisan teacher: A field guide to skillful teaching.

Weddington, NC: Rutherford Learning Group.

Sadler, D. R. (1998). Formative assessment: Revisiting the territory. Assessment in Education: Principles, Policy \& Practice, 5(1), 77-84.

Saettler, L. P. (2004). The evolution of American educational technology. Greenwich, CT. IAP.

Sangra, A., Vlachopoulos, D., \& Cabrera, N. (2012). Building an inclusive definition of e-learning: An approach to the conceptual framework. The International Review of Research in Open and Distributed Learning, 13(2), 145-159.

Schensul, J. J., \& LeCompte, M. D. (2012). Essential ethnographic methods: A mixed methods approach (Vol. 3). Lanham, MD: Rowman Altamira.

Schmidt-Crawford, D. A., Lindstrom, D., \& Thompson, A. D. (2018). Addressing the "why" for integrating technology in teacher preparation. Journal of Digital Learning in Teacher Education, 34(3), 132-133.

Schunk, D. H. (2012). Learning theories an educational perspective $\left(6^{\text {th }}\right.$ ed.). Boston, MA: Pearson Education, Inc.

Schutz, A. (1967). The phenomenology of the social world. Evanston, IL. Northwestern University Press.

Seidman, I. (2013). Interviewing as qualitative research: A guide for researchers in education and the social sciences ( $4^{\text {th }}$ Ed.). New York, NY. Teachers College Press. 
dissertation in practice, B. Jacobs

Shapley, K., Sheehan, D., Maloney, C., \& Caranikas-Walker, F. (2011). Effects of technology immersion on middle school students' learning opportunities and achievement. The Journal of Educational Research, 104(5), 299-315.

Sharples, M., Taylor, J., \& Vavoula, G. (2007). A theory of learning for the mobile age. In R. Andrews \& C. Haythornthwaite (Eds.), The Sage handbook of e-learning research (pp. 221-247). Thousand Oaks, CA: Sage Publications Inc.

Siau, K., Sheng, H., \& Nah, F. H. (2006). Use of a classroom response system to enhance classroom interactivity. IEEE Transactions on Education, 49(3), 398-403.

Siemens, G. (2005). Connectivism: A learning theory for the digital age. International Journal of Instructional Technology and Distance Learning 2(1), 1550-1558 doi: http://er.dut.ac.za/handle/123456789/69.

Simon, F., Malgorzata, K., \& Beatriz, P. (2007). Education and training policy no more failures ten steps to equity in education: Ten steps to equity in education. Paris, [France]. OECD Publishing.

Smidt, H., Thornton, M., \& Abhari, K. (2017). The future of social learning: A novel approach to connectivism. In Proceedings of the 50th Hawaii International Conference on System Sciences. 2116-2125. doi: 10.24251/HICSS.2017.256.

Stone, D. A. (2012). Policy Paradox: The art of political decision making ( $3^{\text {rd }}$ ed.). New York, NY: W.W. Norton \& Company.

Storey, J., Hartley, J., \& Denis, J. L. (Eds.). (2016). The Routledge Companion to Leadership. New York, NY. Taylor \& Francis. 
Stoyanova, N., \& Kommers, P. (2002). Concept mapping as a medium of shared cognition in computer-supported collaborative problem solving. Journal of Interactive Learning Research, 13(1), 111.

Tavangarian, D., Leypold, M. E., Nölting, K., Röser, M., \& Voigt, D. (2004). Is eLearning the solution for individual learning? Electronic Journal of e-Learning, 2(2), 273-280.

Taylor \& Francis Group (2018). Taylor \& Francis Quick Layout Guide. Retrieved from https://authorservices.taylorandfrancis.com/tf_quick_guide/.

Tesch, R. (2013). Qualitative research: Analysis types and software. New York, NY: Routledge.

Thompson, G., \& Glaso, L. (2015). Situational leadership theory: a test from three perspectives. Leadership \& Organization Development Journal, 36(5), 527-544.

Traxler, J. (2007). Defining, discussing and evaluating mobile learning: The moving finger writes and having writ.... The International Review of Research in Open and Distributed Learning, 8(2). doi: http://dx.doi.org/10.19173/irrodl.v8i2.346.

Tyler, T. R., \& De Cremer, D. (2005). Process-based leadership: Fair procedures and reactions to organizational change. The Leadership Quarterly, 16(4), 529-545.

US Department of Education. (March 2015). Science, technology, engineering and math: Education for global leadership. Retrieved from https://www.ed.gov/Stem.

US Department of Education. (February 2017). National Education Technology Plan. Retrieved from https://tech.ed.gov/netp/.

US Department of Education. (January 2016). Budget fact sheet. Retrieved from https://www2.ed.gov/about/overview/budget/budget16/budget-factsheet.pdf. 
dissertation in practice, B. Jacobs

Van der Kleij, F. M., Feskens, R. C., \& Eggen, T. J. (2015). Effects of feedback in a computer-based learning environment on students' learning outcomes: A metaanalysis. Review of educational research, 85(4), 475-511.

Van Laar, E., Van Deursen, A. J., Van Dijk, J. A., \& De Haan, J. (2017). The relation between 21st-century skills and digital skills: A systematic literature review. Computers in Human Behavior, 72, 577-588. doi:10.1016/j.chb.2017. 03.010 .

Van Manen, M. (2015). Researching lived experience: Human science for an action sensitive pedagogy ( $2^{\text {nd }}$ ed.). Walnut Creek, CA. Left Coast Press.

Vaughan, N. D., Cleveland-Innes, M., \& Garrison, D. R. (2013). Teaching in Blended Learning Environments: Creating and Sustaining Communities of Inquiry. Edmonton, AB [Canada]. Athabasca University Press. Retrieved from http://www.ebrary.com.

Vygotsky, L. S. (1978). Mind in society. Cambridge, MA: Harvard University Press. Wang, H., Law, K. S., Hackett, R. D., Wang, D., \& Chen, Z. X. (2005). Leader-member exchange as a mediator of the relationship between transformational leadership and followers' performance and organizational citizenship behavior. Academy of management Journal, 48(3), 420-432.

Wayne, S. J., Shore, L. M., Bommer, W. H., \& Tetrick, L. E. (2002). The role of fair treatment and rewards in perceptions of organizational support and leadermember exchange. Journal of applied psychology, 87(3), 590. 
dissertation in practice, B. Jacobs

Wetzel, K., Foulger, T. S., \& Williams, M. K. (2008). The evolution of the required educational technology course. Journal of Computing in Teacher Education, 25(2), 67-71.

Wheeler, A. H., Goolkasian, P., Baird, A. C., \& Darden, B. V. (1999). Development of the neck pain and disability scale: Item analysis, face, and criterion-related validity. Spine, 24(13), 1290.

Wiliam, D. (2011). Embedded formative assessment. Bloomington, IN: Solution Tree Press.

Winn, W., \& Snyder, D. (1996). Cognitive Perspectives in Psychology. In D. Jonassen and M. Driscoll (Eds.) Handbook of Research for Educational Communications and Technology: A Project of the Association for Educational Communications and Technology (pp. 79-112). New York, NY: Routledge.

Wong, G. K. (2016). A new wave of innovation using mobile learning analytics for flipped classroom. In D. Churchill, J. L, T.K. Chiu, \& B. Fox (Eds.). Mobile Learning Design. Theories and Application (pp. 189-218). [Singapore]: Springer.

Wu, W. H., Wu, Y. C. J., Chen, C. Y., Kao, H. Y., Lin, C. H., \& Huang, S. H. (2012). Review of trends from mobile learning studies: A meta-analysis. Computers \& Education, 59(2), 817-827.

Xie, Y. (2016). On teaching reform of college English based on mobile learning. Revista Ibérica de Sistemas e Tecnologias de Informação, 9, 214-222. 
Yammarino, F. J., \& Dubinsky, A. J. (1994). Transformational leadership theory: Using levels of analysis to determine boundary conditions. Personnel psychology, 47(4), 787-811.

Yelland, N. (2001). Teaching and learning with information and communication technologies (ICT) for numeracy in the early childhood and primary years of schooling. Canberra, [Australia]: Department of Education, Training and Youth Affairs.

Yilmaz, K. (2011). The cognitive perspective on learning: Its theoretical underpinnings and implications for classroom practices. The Clearing House: A Journal of Educational Strategies, Issues and Ideas, 84(5), 204-212.

Yin, R. K. (2003). Case study research: Design and methods (3rd Ed.). Thousand Oaks, CA: Sage Publications, Inc.

Zaccaro, S. J. (2007). Trait-based perspectives of leadership. American Psychologist, 62(1), 6.

Zhu, W., Avolio, B. J., Riggio, R. E., \& Sosik, J. J. (2011). The effect of authentic transformational leadership on follower and group ethics. The Leadership Quarterly, 22(5), 801-817. 


\section{APPENDIX A}

\section{Informed Consent}

1. Gatekeeper Permission for Administrator and Educator Participation Letter

2. Gatekeeper Permission for Administrator and Educator Participation

3. Letter of Informed Consent - Educator Participant

4. Informed Consent from Leader Participant 
dissertation in practice, B. Jacobs

\section{Gatekeeper Permission for Administrator and Educator Participation Letter} Lee's Summit R-7 School District

Dear <name>,

I would like to request your permission to invite applicable educators in your program to participate in a research study entitled: An examination of technology-based formative assessment feedback and the impact on student learning. I am examining the reported gains in student achievement using technology-based formative assessment tools and applications to provide feedback to teachers and students to inform instructional practice and increase student learning. Interviews and focus groups will be coded to determine the extent to which technologyenhanced formative assessment has been impacted by professional development for teachers, instructional design, and student learning within $7^{\text {th }}$ and $8^{\text {th }}$-grade mathematics curriculum. The information gathered should be beneficial to the district, specifically to teachers, building administrators, and district-level employees who support instruction and learning within the classroom. This study is part of my dissertation research for a doctoral degree in Educational Leadership and Policy Analysis from the University of Missouri-Columbia.

For the study, building administrators and district technology administrators will be asked to complete a qualitative interview. One administrator from each middle school and the Executive Director/Director of Technology and will be asked to participate. Additionally, a selection of teachers, Instructional Technology Specialists, K-12 Curriculum Coordinators, and Tier 1 Instructional Specialists will be asked to participate in a qualitative focus group. No personal or identifying information will be collected from these educators. I am seeking your permission as the Superintendent of the Lees Summit School District to contact the administrators and educators of the district for their participation in this study. A copy of the interview protocol and informed consent forms are attached for your review.

Participation in the study is completely voluntary. The participants may withdraw from participation at any time they wish without penalty, including in the middle of or after completion of the interview or focus groups. Participants' answers will remain confidential, anonymous, and separate from any identifying information. The researcher will not list any names of participants in his dissertation or any future publications of this study.

Please do not hesitate to contact me with any questions or concerns about participation either by phone at (816) 210-0310 or by electronic mail at baj79e@ mail.missouri.edu. In addition, you are also welcome to contact the dissertation advisor for this research study, Dr. Barbara Martin, who can be reached at 660-543-8823 or by email at bmartin@ucmo.edu.

If you choose to allow me to contact administrators and educators regarding participation in this study, please complete the attached permission form. You should retain a copy of this letter and your written consent for future reference.

Thank you for your time and consideration.

Sincerely,

Brett Jacobs

Doctoral Candidate 


\section{Gatekeeper Permission for Administrator and Educator Participation}

$\mathrm{I}$, grant permission for administrators and educators within the Lees Summit School District to be contacted to participate in the study An examination of technology-based formative assessment feedback and the impact on student learning conducted by Brett Jacobs, doctoral candidate at the University of Missouri.

By signing this permission form, I understand that the following safeguards are in place to protect faculty choosing to participate:

- All participation is voluntary, and may be withdrawn at any point before culmination of the study.

- All responses will be used for dissertation research and for potential future journal publications.

- All identities will be kept confidential in all phases of the research.

- An interview will occur with each administrator, director, or teacher either inperson or via videoconference, lasting approximately one hour in length.

Please keep the consent letter and a copy of the signed consent form for your records. If you choose to grant permission for educators in your school district to participate in this study, please complete this Administrative Permission for Program Participation Form, please return it to Brett Jacobs as soon as possible.

I have read the material above, and any questions that I have posed have been answered to my satisfaction. I grant permission for administrators and educators in my program to be contacted and invited to participate in this study.

Signed: Date:

Title/Position:

Institution:

Please return to: Brett Jacobs, 325 SE Saratoga Drive, Blue Springs, MO, 64014 Cell Phone: 816-210-0310 Email: baj79e@mail.missouri.edu 
APPENDIX B

IRB and Gatekeeper Approval

1. IRB Approval Letter - University of Missouri

2. Approval letter from participating district 
Institutional Review Board University of Missouri-Columbia FWA Number 000sit6

IRE Registration Numbers 00000731,00009014
442 MeAcynolds Hall

Columbin, MO 65211

573823181

irbômissouri.edu

January 22, 2019

Principal Investigator: Arett A Jacobs (MU Student)

Department: Educational Leadership-EDD

Your IPB Application to project entitled AN EXMMINATION OF TECHNOLOGY-BASED FORMATVE ASSESSMENT FEFDENCK AND THE MMPACT ON STUDENT LEARNING wa reviewed and approwed by the MU Institutional Review Board acoording to the terms and conditions described below:

$\begin{array}{ll}\text { IAB Ptoject Number } & 2013555 \\ \text { IRB Beview Number } & 24355 \\ \text { Initial Application Approval Date } & \text { January 22, 2019 } \\ \text { IRB Expiration Date } & \text { January 22, 2020 } \\ \text { Level of Review } & \text { Exempt } \\ \text { Project Status } & \text { Active - Exempt } \\ \text { Exempt Categories (Revised Common } & 45 \text { CFR 46.104d(1) } \\ \text { Hule) } & \\ \text { Hisk Level } & \text { Minimal Risk }\end{array}$

The principal investigator (PI) is responsible for all aspects and conduct of this study. The PI must comply with the following conditions of the approval:

1. No subjects may be involved in any study procedure prior to the IRB approval date or after the expiration date.

2. All changes must be IRB approved prior to implementation utilizing the Exempt Amendment Form.

3. The Annual Exempt Form must be submitted to the IRB for review and approval at least 30 days prior to the project expiration date to keep the study active or to close it.

4. Maintain all research records for a period of seven years from the project completion date.

If you have any questions, please contact the IRB at 572-882-3131 or irbofmissouri.edu.

Thank you,

MU Institutional Heview Hoard 


\section{APPENDIX C \\ Informed Consent - Educator Participant}

$\mathrm{I}$, , agree to participate in the

study: An examination of technology-based formative assessment feedback and the impact on student learning conducted by Brett Jacobs, doctoral candidate at the University of Missouri. I understand the following:

- My participation is voluntary, and may be withdrawn at any point before culmination of the study.

- My responses will be used for dissertation research and for potential future journal publications.

- My identity will be kept confidential in all phases of the research.

- A focus group will occur either in-person or via video conference at a mutually agreed upon time, lasting approximately one hour in length.

Please keep the consent letter and a copy of the signed consent form for your records. If you choose to participate in this study, please complete the attached signed consent form, seal it in the enclosed envelope, and return to Brett Jacobs as soon as possible. Please to be sure and include contact information so interview plans can be made and communicated to you.

I have read the material above, and any questions that I have posed have been answered to my satisfaction. I voluntarily agree to participate in this study.

Signed:

Date:

Title/Position:

\section{Contact Information:}

Phone (circle one)

WORK HOME

CELL

Best time for contact:

E-mail:

Please return to: Brett Jacobs, 325 SE Saratoga Drive, Blue Springs, MO, 64014 Cell Phone: 816-210-0310 Email: baj79e@mail.missouri.edu 


\section{APPENDIX D}

\section{Informed Consent - Leader Participant}

$\mathrm{I}$, , agree to participate in the

study: An examination of technology-based formative assessment feedback and the impact on student learning conducted by Brett Jacobs, doctoral candidate at the University of Missouri. I understand the following:

- My participation is voluntary, and may be withdrawn at any point before culmination of the study.

- My responses will be used for dissertation research and for potential future journal publications.

- My identity will be kept confidential in all phases of the research.

- An interview will occur either in-person or via video conference at a mutually agreed upon time, lasting approximately one hour in length.

Please keep the consent letter and a copy of the signed consent form for your records. If you choose to participate in this study, please complete the attached signed consent form, seal it in the enclosed envelope, and return to Brett Jacobs as soon as possible. Please to be sure and include contact information so interview plans can be made and communicated to you.

I have read the material above, and any questions that I have posed have been answered to my satisfaction. I voluntarily agree to participate in this study.

Signed:

Date:

Title/Position:

\section{Contact Information:}

Phone (circle one)

WORK

HOME CELL

Best time for contact:

E-mail:

Please return to: Brett Jacobs, 325 SE Saratoga Drive, Blue Springs, MO, 64014 Cell Phone: 816-210-0310 Email: baj79e@mail.missouri.edu 
dissertation in practice, B. Jacobs

\section{APPENDIX E}

Focus Group and Interview Protocols

Focus Group Protocol: Classroom Teacher

Focus Group Protocol: Curriculum and Instruction Team

Interview Protocol: Building Administrator

Interview Protocol: District Administrator 
Focus Group Protocol: Classroom Teachers

Date:

Start Time:

Introduction:

Welcome. Thank you for taking the time to discuss your opinions and observations regarding technology-based formative assessment feedback. My name is Brett Jacobs, and I will serve as the moderator for today's focus group. The purpose of today's discussion is to get information from you about the impact of specific types of formative assessment feedback on classroom instruction and student achievement in reference to learning objectives outlined by your district.

Please remember, there are no right or wrong answers but rather differing points of view. Feel free to share your point of view even if it differs from what others have said. If you want to follow-up on something that someone has said, you want to agree, disagree or give an example, feel free to do that. This is meant to be more of a conversation among yourselves, so don't feel like you have to respond to me all of the time. I am here to ask questions, listen and make sure everyone has a chance to share. I am interested in hearing from each of you. Please speak up and remember only one person should talk at a time.

Our session will last about an hour, and we will not be taking a formal break. Feel free to leave the table for any reason if you need to. While the nature of a focus group makes it impossible to provide complete anonymity, the evaluator will maintain your confidentiality during future reporting. No names will be included in any reports. Let us begin by going around the room and finding out more about each other.

\begin{tabular}{|c|c|}
\hline Questions & Information \\
\hline 1. Please tell me your name and how long you have been a teacher. & $\begin{array}{l}\text { Learn } \\
\text { about } \\
\text { participants }\end{array}$ \\
\hline $\begin{array}{l}\text { 2. Please explain your learning community, specifically concerning } \\
\text { teacher access to technology, student access to technology, resources } \\
\text { allotted to technology instruction, hardware/software programs, etc. } \\
\text { Probe: } \\
\text { Please describe your philosophy (personal, district, or a combination } \\
\text { there of) regarding technology access, instruction, assessment, etc.? } \\
\text { Do you feel you have access or means to obtain technology based } \\
\text { resources to support the teaching and learning philosophy you described? } \\
\text { What types of training or professional development have you sought out, } \\
\text { or has the district offered, regarding formative assessment and/or } \\
\text { formative assessment utilizing technology? }\end{array}$ & $\begin{array}{c}\text { Transition } \\
\text { Question } \\
\text { Obtain } \\
\text { information } \\
\text { about } \\
\text { District } \\
\text { Technology } \\
\text { Vision }\end{array}$ \\
\hline $\begin{array}{l}\text { 3. Before technology apps were accessible, describe how often you } \\
\text { provided feedback to students about their progress on specific learning } \\
\text { objectives? }\end{array}$ & Q2 \& Q3 \\
\hline
\end{tabular}




\begin{tabular}{|c|c|}
\hline $\begin{array}{l}\text { Probe: } \\
\text { How often was feedback an imbedded part of your learning cycle (within } \\
\text { the class period, daily, weekly, etc)? } \\
\text { What types of feedback or data did you provide students within the } \\
\text { teaching and learning process? }\end{array}$ & \\
\hline $\begin{array}{l}\text { 4. What does formative assessment mean within the context of your } \\
\text { classroom? } \\
\text { Probe: } \\
\text { How often do you incorporate formative assessment feedback within } \\
\text { your lessons? } \\
\text { How often do you utilize technology to formatively assess student } \\
\text { learning? } \\
\text { Describe how/whether you utilize information gathered from formative } \\
\text { assessments to change/alter instruction. }\end{array}$ & Q1, Q2 \\
\hline $\begin{array}{l}\text { 5. Are there specific technology applications (ALEKS, IXL, Schoology, } \\
\text { etc.) that you use to formatively assess student learning? } \\
\text { Probe: } \\
\text { Describe how often you use, or encourage students to use, these } \\
\text { applications. Are there some that you utilize more frequently than } \\
\text { others? } \\
\text { Please describe how/whether the utilization of these applications effects } \\
\text { learning opportunities for students inside the classroom, outside of the } \\
\text { classroom setting, or both. }\end{array}$ & Q1, Q3 \\
\hline $\begin{array}{l}\text { 6. Describe how technology-based formative assessment tools have } \\
\text { impacted data collection and dissemination within your classroom? } \\
\text { Probes: } \\
\text { How, if at all, has incorporating technology in your data collection and } \\
\text { feedback process impacted the learning within your classroom? }\end{array}$ & $\mathrm{Q} 1, \mathrm{Q} 2, \mathrm{Q} 3$ \\
\hline $\begin{array}{l}\text { 7. Has incorporating technology as a part of the formative assessment } \\
\text { and feedback process had an impact on your planning or instruction? If } \\
\text { so, what kind of impact? } \\
\text { Probe: } \\
\text { Do you feel that technology impacts how much time you spend } \\
\text { instructionally on a topic or objective? }\end{array}$ & Q1, Q3 \\
\hline $\begin{array}{l}\text { 8. How, if at all, does the accessible feedback and reflection data impact } \\
\text { the way students approach learning? } \\
\text { Probe: } \\
\text { Does providing technology-based formative assessment feedback to } \\
\text { students impact student learning? If so, how? }\end{array}$ & Q1, Q3 \\
\hline
\end{tabular}


9. What would you like to add to the discussion you feel is important that $\mathrm{Q1,}$ Q2, Q3 I did not ask about?

I would like to take this opportunity to thank you for your time and participation in this research project. When the research is completed, you will have the opportunity to read the results of the research. 
Focus Group: Curriculum and Instruction Team

Date:

Start Time:

Introduction:

Welcome. Thank you for taking the time to discuss your opinions and observations regarding technology-based formative assessment feedback. My name is Brett Jacobs, and I will serve as the moderator for today's focus group. The purpose of today's discussion is to get information from you about the impact of specific types of formative assessment feedback on classroom instruction and student achievement about learning objectives outlined by your district.

Please remember, there are no right or wrong answers but rather differing points of view. Feel free to share your point of view even if it differs from what others have said. If you want to follow-up on something that someone has said, you want to agree, disagree or give an example, feel free to do that. This is meant to be more of a conversation among yourselves, so don't feel like you have to respond to me all of the time. I am here to ask questions, listen and make sure everyone has a chance to share. I am interested in hearing from each of you. Please speak up and remember only one person should talk at a time.

Our session will last about an hour, and we will not be taking a formal break. Feel free to leave the table for any reason if you need to. While the nature of a focus group makes it impossible to provide complete anonymity, the evaluator will maintain your confidentiality during future reporting. No names will be included in any reports. Let us begin by going around the room and finding out more about each other.

\begin{tabular}{|c|c|}
\hline Questions & Information \\
\hline $\begin{array}{l}\text { 1. Please tell me your names and describe your experiences in } \\
\text { education (teaching experience, current position and how long, } \\
\text { role in the district, etc.) }\end{array}$ & $\begin{array}{l}\text { Learn } \\
\text { about } \\
\text { participants }\end{array}$ \\
\hline $\begin{array}{l}\text { 2. Please explain your learning community, specifically about teacher } \\
\text { access to technology, student access to mobile devices, resources allotted } \\
\text { to technology instruction (hardware/software applications programs, etc.) } \\
\text { Probe: } \\
\text { - Please describe your philosophy (personal, district, or a } \\
\text { combination there of) regarding technology access, instruction, } \\
\text { assessment, etc.? i.e. please describe the essential elements of } \\
\text { successful technology usage? } \\
\text { - What is the role of this team regarding technology access and } \\
\text { integration - i.e. decisions regarding teacher tools/applications - } \\
\text { student usage relative to meeting designated learning targets? } \\
\text { - How often do you meet as a district level instructional team? } \\
\text { How often do you, within your roles, meet with instructional }\end{array}$ & $\begin{array}{c}\text { Transition } \\
\text { Question } \\
\text { Obtain } \\
\text { information } \\
\text { about } \\
\text { District } \\
\text { Technology } \\
\text { Vision }\end{array}$ \\
\hline
\end{tabular}




\begin{tabular}{|c|c|}
\hline $\begin{array}{l}\text { teams within the building (i.e. administrators and/or teaching } \\
\text { staff) curriculum and/or instructional practice? }\end{array}$ & \\
\hline $\begin{array}{l}\text { 3. Before technology apps were readily accessible, describe the } \\
\text { curricular or instructional value the district placed on the use of } \\
\text { formative assessment? } \\
\text { Probe: } \\
\text { - Do you feel the district values and encourages the use of } \\
\text { formative assessment feedback strategies? } \\
\text { - Describe the benefits of utilizing formative assessment within } \\
\text { teaching and learning? } \\
\text { - What are some examples of how your instructional team } \\
\text { promotes formative assessment within teaching and learning? }\end{array}$ & Q1, Q3 \\
\hline $\begin{array}{l}\text { 4. The middle school math teachers in this district utilize, either } \\
\text { currently or in the past, technology applications such as IXL, ALEKS, } \\
\text { Kahoot, Google applications and Schoology. Can you confirm that these } \\
\text { are the primary applications utilized consistently by math teachers within } \\
\text { the teaching and learning process within the district? } \\
\text { Probe: } \\
\text { - Are there other applications that are used and embedded } \\
\text { consistently within the district? Can you give some examples? } \\
\text { - When considering technology-based formative assessment, what } \\
\text { key characteristics or functions do you consider significant } \\
\text { applications or components within a software program that } \\
\text { justifies promoting that program to teachers? }\end{array}$ & $\mathrm{Q} 2, \mathrm{Q} 3$ \\
\hline $\begin{array}{l}\text { 5. Describe the impact technology-based formative assessment tools } \\
\text { have on data collection within the classrooms you observe or the } \\
\text { administrators/teachers you meet with. } \\
\text { Probe: } \\
\text { - Have technology applications influenced the way that teachers } \\
\text { provide, and/or students receive, feedback? } \\
\text { - Have technology applications affected the frequency of } \\
\text { feedback? } \\
\text { - Describe how the way in which feedback is gathered/received } \\
\text { effects on student learning within middle school mathematics? } \\
\text { - Describe how the frequency of feedback, specifically technology- } \\
\text { based formative assessment feedback, effects on student learning } \\
\text { within middle school mathematics? }\end{array}$ & Q2, Q3 \\
\hline
\end{tabular}



6. How do technology-based tools or applications impact the way teachers plan for instruction?
Probes:
- Does the feedback teachers or students receive utilizing technology-based applications allow for an increased level of instruction?
- Do technology based applications increase the level of student- student interaction and feedback students receive from each other?
- Does technology allow for broader or deeper understanding of learning objectives? If so, how? (i.e. more content, deeper learning within learning targets, adjustments to instruction, etc.)
- Do you feel that technology impacts how much time teachers spend instructionally on a topic or objective?

7. Describe how, if at all, the accessibility of technology tools and applications, specifically those supported by the district (IXL, ALEKS, Google Apps, Schoology) impact student learning opportunities outside of the classroom?

Probe:

- How do those specific technology tools and applications impact the way student's access information within and beyond the classroom?

- How do those technology tools and applications impact the way student's receive feedback on their progress and learning within and beyond the classroom?

- Do technology applications impact the way students analyze their own growth and progress on specific learning targets? If so, describe the way the district supported applications allow this to occur.

8. Do you, individually or as a team, analyze student achievement data when developing or modifying district curriculum? Probe:

- If so, what types of data do you analyze?

- How does that data impact the vision of your team? (i.e. how does this help you support instruction and student learning)

- What impact has the incorporation of technology had on district curriculum or instructional practice?

- Has incorporating technology as a part of formative assessment made an impact on student achievement?
Q1, Q3

Q1, Q3 


\begin{tabular}{|c|c|}
\hline $\begin{array}{l}\text { 9. Based on your observations, do teachers primarily utilize technology } \\
\text { tools and technology-based applications to Substitute for, Augment, } \\
\text { Modify, or Redefine learning opportunities? } \\
\text { Probe: } \\
\text { - As such, which of these terms - Substitution, Augmentation, } \\
\text { Modification, or Redefinition - best describe how technology/, } \\
\text { mobile learning devices and technology-based applications have } \\
\text { impacted the teaching and learning process? }\end{array}$ & Q1, Q3 \\
\hline $\begin{array}{l}\text { 10. What elements of the training and/or professional development } \\
\text { opportunities you provide do specific to technology-based formative } \\
\text { assessment apps benefits teachers instructionally and/or student learning? } \\
\text { How? } \\
\text { Probe: } \\
\text { - How often do teachers receive training and/or professional } \\
\text { development related to the applications you find are most } \\
\text { effective for formative assessment within instruction? } \\
\text { - What feedback have you received from administrators and/or } \\
\text { teachers regarding the usefulness of these professional } \\
\text { development sessions? } \\
\text { - Can teachers elect to participate in additional professional } \\
\text { development outside of the district utilizing district resources? }\end{array}$ & Q1, Q3 \\
\hline $\begin{array}{l}\text { 11. What would you like to add to the discussion you feel is important } \\
\text { that I did not ask about? }\end{array}$ & \\
\hline
\end{tabular}

Thank you for your time and participation. 
Interview Protocol: Building Administrator

Name of participant:

Site:

Date:

Start Time:

Introduction: Good afternoon. Thank you for taking the time to answer my questions focusing on your experiences with technology-based formative assessment. The purpose of today's discussion is to get information from you about the impact of technologybased formative assessment on instruction, and ultimately student achievement. My name is Brett Jacobs, and I will be conducting the interview. In order to ensure accuracy, I will be audio taping the interview.

Remember, there are no right or wrong answers. If you want to follow-up on a question or give an example, feel free to do so. I want this to be more of a conversation between professionals.

Our session will last about one and a half to two hours and we will not be taking a formal break. Please let me know if you need to leave the table for any reason. Let us begin by finding out more about each other.

\begin{tabular}{|c|c|}
\hline Questions & Information \\
\hline $\begin{array}{l}\text { 1. Please tell me your name and describe your experiences in } \\
\text { education (teaching experience, current position and how long, } \\
\text { etc.) }\end{array}$ & $\begin{array}{l}\text { Learn } \\
\text { about } \\
\text { participants }\end{array}$ \\
\hline $\begin{array}{l}\text { 2. Please explain your learning community, specifically in regard to } \\
\text { teacher access to technology, student access to technology, resources } \\
\text { allotted to technology instruction, hardware/software programs, etc. } \\
\text { Probe: } \\
\text { - What is your role regarding technology access and } \\
\text { integration? } \\
\text { - How often do you meet with teachers regarding curriculum } \\
\text { and/or instructional practice? }\end{array}$ & $\begin{array}{l}\text { Transition } \\
\text { Question } \\
\text { Obtain } \\
\text { information } \\
\text { about } \\
\text { District } \\
\text { Technology } \\
\text { Vision }\end{array}$ \\
\hline $\begin{array}{l}\text { 3. What does formative assessment mean within the context of teaching } \\
\text { and learning within your building? } \\
\text { Probe: } \\
\text { students? } \\
\text { - What are your expectations with regard to how information } \\
\text { gathered from formative assessments impact instructional design } \\
\text { and learning opportunities? } \\
\text { - How often do you provide feedback to teachers on their use } \\
\text { of formative assessment strategies? }\end{array}$ & $\mathrm{Q} 1, \mathrm{Q} 2$ \\
\hline
\end{tabular}




\begin{tabular}{|c|c|}
\hline $\begin{array}{l}\text { 4. Would you describe the benefits or drawbacks if any, of utilizing } \\
\text { formative assessment within teaching and learning? } \\
\text { Probe: } \\
\text { - How does utilizing verbal feedback differ from assigned } \\
\text { tasks? } \\
\text { - How do teachers utilize information from formative } \\
\text { assessment feedback to adjust learning opportunities? } \\
\text { - How do students utilize information from formative } \\
\text { assessment feedback within the cycle of learning, within and } \\
\text { outside of the classroom. }\end{array}$ & Q1, Q3 \\
\hline $\begin{array}{l}\text { 5. Describe how, if at all, technology-based formative assessment tools } \\
\text { have impacted data collection within the classrooms you observe or the } \\
\text { teachers you meet with. } \\
\text { Probes: } \\
\text { - Have technology apps influenced the way that teachers } \\
\text { provide feedback to students? } \\
\text { - Have technology apps affected the frequency of feedback } \\
\text { students? }\end{array}$ & Q2, Q3 \\
\hline $\begin{array}{l}\text { 6. . Do you feel that technology impacts how much time teachers spend } \\
\text { instructionally on a topic or objective? } \\
\text { Probe: } \\
\text { - Does incorporating technology provide any benefits } \\
\text { regarding the timeliness and availability of feedback? } \\
\text { - Does technology allow for less time to be spent on a learning } \\
\text { objective? } \\
\text { - Does technology allow for alternate instructional strategies to } \\
\text { be used to preserve class time? }\end{array}$ & Q2, Q3 \\
\hline $\begin{array}{l}\text { 7. Do students have access to their own formative assessment data? If so, } \\
\text { what kinds of data do students have access to? } \\
\text { Probe: } \\
\text { - In general, how does feedback from formative assessment } \\
\text { data impact student learning on specific learning targets? } \\
\text { - Do technology applications impact the way students analyze } \\
\text { their own progress? } \\
\text { - How do outside learning opportunities effect the feedback } \\
\text { students obtain, and utilize, to meet desired learning objectives? }\end{array}$ & $\mathrm{Q} 1, \mathrm{Q} 2, \mathrm{Q}$ \\
\hline $\begin{array}{l}\text { 8. Do you, individually or as an instructional team, analyze student } \\
\text { achievement data? } \\
\text { Probe: } \\
\text { - If so, what types of data do you analyze? } \\
\text { - How does that data impact the way your instructional team } \\
\text { shapes learning opportunities for teachers and students? } \\
\text { - What impact has the incorporation of technology had on teaching } \\
\text { and learning in your building? }\end{array}$ & Q1, Q3 \\
\hline
\end{tabular}


- Does incorporating technology as a part of formative assessment make an impact on student achievement (classroom, district, statewide)?

9. Do you feel that training and/or professional development specific to $\quad$ Q2, Q3 technology-based formative assessment apps benefits teachers instructionally and/or student learning? Why or why not?

Probe:

- How often do you provide training and/or professional development related to the applications you find are most effective for formative assessment within instruction?

10. What would you like to add to the discussion you feel is important that I did not ask about?

Thank you for your time and participation. 
Interview Protocol: District Administrator

Name of participant:

Role of participant:

Date:

Start Time:

Introduction: Good afternoon. Thank you for taking the time to answer my questions focusing on your experiences with technology-based formative assessment. The purpose of today's discussion is to get information from you about the impact of technologybased formative assessment on instruction, and ultimately student achievement. My name is Brett Jacobs, and I will be conducting the interview. In order to ensure accuracy, I will be audio taping the interview.

Remember, there are no right or wrong answers. If you want to follow-up on a question or give an example, feel free to do so. I want this to be more of a conversation between professionals.

Our session will last about one and a half to two hours and we will not be taking a formal break. Please let me know if you need to leave the table for any reason. Let us begin by finding out more about each other.

\begin{tabular}{|c|c|}
\hline Questions & Information \\
\hline $\begin{array}{l}\text { 1. Please tell me your name and describe your experiences in education } \\
\text { (teaching experience, leadership experience, current position and how } \\
\text { long, etc.) }\end{array}$ & $\begin{array}{l}\text { Learn } \\
\text { about } \\
\text { participants }\end{array}$ \\
\hline $\begin{array}{l}\text { 2. Please explain your district learning community, specifically in regard } \\
\text { to teacher access to technology applications, student access to mobile } \\
\text { learning devices, resources allotted to technology instruction, } \\
\text { hardware/software programs, etc. } \\
\text { Probe: } \\
\text { - What is your role regarding technology access and integration? } \\
\text { - How often do you meet with your instructional team regarding } \\
\text { infusion of technology within curriculum and/or instructional } \\
\text { practice? }\end{array}$ & $\begin{array}{c}\text { Transition } \\
\text { Question } \\
\text { Obtain } \\
\text { information } \\
\text { about } \\
\text { District } \\
\text { Technology } \\
\text { Vision }\end{array}$ \\
\hline $\begin{array}{l}\text { 3. Does the district have a process for selecting technology-based } \\
\text { formative assessment tools? } \\
\text { Probes: } \\
\text { - If so, what are the primary factors considered during selection? } \\
\text { - Does the frequency, quality, or autonomy of feedback impact the } \\
\text { way you select technology based applications? }\end{array}$ & Q1, Q2 \\
\hline $\begin{array}{l}\text { 4. What does formative assessment mean within the context of teaching } \\
\text { and learning within the district? Is it an expectation that teachers embed } \\
\text { formative assessment within teaching and learning? }\end{array}$ & Q1, Q3 \\
\hline
\end{tabular}


Probe:

- Would you describe the benefits, if any, of utilizing formative assessment within teaching and learning?

- What role does formative assessment play within teaching and learning process?

- Does the district implement and monitor strategies of formative assessment within instruction?

5. Describe how, if at all, technology-based formative assessment tools have impacted the way student performance feedback is utilized within the classrooms you or your team observe, or the teachers you meet with. Probe:

- Does incorporating technology provide any benefits regarding the availability and use of feedback?

- Does incorporating technology-based applications increase the frequency of formative assessment feedback within the teaching and learning process?

6. At the middle school level, are there specific technology-based applications where students have access to their own formative assessment data? If so, what kinds of data do students have access to? Probes:

- Does technology have an impact on the amount of time it takes to provide feedback to students?

- Do technology apps impact the way students analyze their own progress?

7. Do you feel that training and/or professional development specific to Q1, Q2, Q3 technology-based formative assessment apps benefits teacher instruction and/or student learning? Please explain.

Probe:

- How often does the district instructional team provide training and/or professional development related to the applications such as IXL, ALEKS, or Schoology specific to formative assessment within teaching and learning?

8. How have technology-based applications, specifically IXL, ALEKS, and Schoology, impacted the way teachers address learner objectives? Probes:

- Do you feel that technology impacts how much instructional time teachers spend on a topic or objective?

- Does technology impact the breadth or depth of topics teachers can cover within allowed instructional time? 
- Which of these terms - Substitution, Augmentation, Modification, or Redefinition - best describe how technology/, mobile learning devices and technology-based applications have impacted the teaching and learning process?

9. Has incorporating technology tools as a part of formative assessment Q2, Q4 made an impact on student learning and achievement?

Probe:

- What other variables should be considered when evaluating the effect technology-based formative assessment on student learning?

- Are there specific benefits, not previously discussed, which technology provides to the learning process that could not be done without technology?

10. What would you like to add to the discussion you feel is important that I did not ask about?

Thank you for your time and participation. 
APPENDIX F

Document Analysis Protocol

\begin{tabular}{|l|l|}
\hline Date: & Title of document: \\
\hline Site: & Obtained from: \\
\hline Why was this document created? & Who is the intended audience? \\
$\begin{array}{l}\text { How does the document reflect } \\
\text { organizational culture? }\end{array}$ & $\begin{array}{l}\text { Do parts of the document relate technology- } \\
\text { based formative assessment and impact on } \\
\text { student learning? }\end{array}$ \\
$\begin{array}{ll}\text { a) Norms: } \\
\text { c) Artifacts: }\end{array}$ & a) Impact of technology: \\
b) Assumptions, Values \& Beliefs: & b) Impact of formative assessment: \\
\hline
\end{tabular}


dissertation in practice, B. Jacobs

APPENDIX G

Observation Protocol Form

Date of Observation

Contact Name

Start Time

End Time

Location

Description of observed area:

Field Notes: 


\section{APPENDIX H}

\section{Data Codes}

Leader A District Leader Participant 1

Leader B District Leader Participant 2

Principal A Interview with Principal 1

Principal B Interview with Principal 2

Principal C Interview with Principal 2

FGA Focus Group Site A

Teacher A Teacher Participant 1

Teacher B Teacher Participant 2

Teacher C Teacher Participant 3

Teacher D Teacher Participant 4

Teacher E Teacher Participant 5

FGB Focus Group Site B

Teacher F Teacher Participant 1

Teacher G Teacher Participant 2

Teacher H Teacher Participant 3

Teacher I Teacher Participant 4

Teacher J Teacher Participant 5

FGC Focus Group Site C

Teacher L Teacher Participant 1

Teacher M Teacher Participant 2

Teacher N Teacher Participant 3

Teacher O Teacher Participant 4

Teacher P Teacher Participant 5

FGD Focus Group District Instructional Team

Specialist A Focus Group 3 Participant 1

Specialist B Focus Group 3 Participant 2

Specialist C Focus Group 3 Participant 3

Specialist D Focus Group 3 Participant 4

Specialist E Focus Group 3 Participant 5

Specialist F Focus Group 3 Participant 6 
VITA

Brett Jacobs was born in Wichita, KS to Stephen and Mary Jacobs. He graduated in 1996 from Blue Springs High School in Blue Springs, MO. In 2000, he received a Bachelor of Science in Secondary Education from the University of Missouri Columbia. He later earned a Masters in Educational Leadership and Policy Analysis, followed by a Doctorate in Educational Leadership and Policy Analysis from the University of Missouri - Columbia in 2019.

After beginning his career in the Blue Springs School District teaching high school mathematics for two years, Brett moved to the Independence School district and transitioned to middle-level education. In Independence, Brett spent 5 years teaching $8^{\text {th }}$ grade mathematics and was the lead teacher of his department and grade-level. After his time as a mathematics teacher, Brett transition into his first administrative role as the assistant principal of James Bridger Middle School, in Independence, Missouri. In 2008, he seized the opportunity to re-open Clifford H. Nowlin Middle School when the district absorbed schools from the Kansas City School District via annexation. Since 2013, he has been an assistant principal and activities director at Bernard C. Campbell Middle School in the Lee's Summit School District in Lee's Summit, Missouri.

Brett served on district instructional teams to create and align mathematics curriculum to state standards, and chaired committees designated to align to National Education Technology Standards (referred to currently as the ISTE standards). Through these experiences, Brett developed a passion for technology integration and education, specifically within mathematics. 
dissertation in practice, B. Jacobs

Brett currently resides in Blue Springs, Missouri, with his wife Sandra Jacobs, and his four children Heidi, Connor, Stephen and Kollin. 